CIRJE-F-815

\title{
On Approximation of the Solutions to Partial Differential Equations in Finance
}

\author{
Akihiko Takahashi \\ University of Tokyo \\ Toshihiro Yamada \\ Mitsubishi UFJ Trust Investment Technology Institute Co.,Ltd.
}

August 2011; Revised in January 2012

CIRJE Discussion Papers can be downloaded without charge from:

$\underline{\text { http://www.cirje.e.u-tokyo.ac.jp/research/03research02dp.html }}$

Discussion Papers are a series of manuscripts in their draft form. They are not intended for circulation or distribution except as indicated by the author. For that reason Discussion Papers may not be reproduced or distributed without the written consent of the author. 


\title{
On Approximation of the Solutions to Partial Differential Equations in Finance *
}

\author{
Akihiko Takahashi ${ }^{\dagger}$ and Toshihiro Yamada ${ }^{\ddagger}$ \\ First Version: November 3, 2010, Second Version: July 5, 2011, Third Version: August 22, \\ 2011, Forth Version: December 20, 2011, This Version: January 12
}

\begin{abstract}
This paper proposes a general approximation method for the solutions to second-order parabolic partial differential equations (PDEs) widely used in finance through an extension of Léandre's approach(Léandre $(2006,2008))$ and the Bismut identiy(e.g. chapter IX-7 of Malliavin (1997)) in Malliavin calculus. We show two types of its applications, new approximations of derivatives prices and short-time asymptotic expansions of the heat kernel. In particular, we provide new approximation formulas for plain-vanilla and barrier option prices under stochastic volatility models. We also derive short-time asymptotic expansions of the heat kernel under general time-homogenous local volatility and local-stochastic volatility models in finance which include Heston (Heston (1993)) and $(\lambda$-)SABR models (Hagan et.al. (2002), Labordere (2008)) as special cases. Some numerical examples are shown.
\end{abstract}

Keywords: Barrier Options, Knock-out options, SABR model, $\lambda$-)SABR models, Heston model, Short time asymptotics, Heat kernel expansions, Malliavin calculus, Bismut indentity, Stochastic volatility, Local volatility, Integration-by-parts, Semigroup, Derivatives pricing

\section{Introduction}

This paper proposes a new method for the approximation to the solutions of second-order parabolic partial differential equations (PDEs), which has been widely used for pricing and hedging derivatives in finance since Black-Scholes (1973) and Merton (1973).

In particular, we derive an approximation formula as Theorem 3.1 based on an asymptotic expansion of the solutions to the second-order parabolic PDEs by Léandre's Approach (Léandre $(2006,2008)$ ) and an application of Malliavin calculus effectively: the approximation formula is derived through an extension of Léandre's "elementary integration by parts formula" (Theorem 2.2 in Léandre (2006)) presented in Proposition 3.1, and an application of the Bismut identity (e.g. chapter IX-7 of Malliavin (1997)). Also, this derivation can be regarded as an extension of the PDE weight method in Malliaivn-Thalmaier (2006) to an asymptotic expansion of the solutions of the PDEs.

Moreover, our method has an advantage in a sense that our computational scheme can be applied to various diffusion models in a unified way to obtaining derivatives' prices and Greeks under various (multidimensional) diffusion models. Especially, it is stressed that as an application we derive a new approximation formula for pricing barrier options under a stochastic volatility model, a SABR model, where our formula is obtained by an expansion of the well-known barrier option formula under Black-Scholes model. Note that because SABR model has no mean-reverting component in the volatility process, the fast mean-reverting asymptotic analysis by Fouque et al.(2000a,b) and Ilhan et al.(2004) seems not applicable to this model.

In addition, we apply this method to deriving a short-time asymptotic expansion of the heat kernel under the general diffusion setting which includes general time-homogenous local volatility, Heston and $(\lambda-)$ SABR

*The previous version is entitled "On the Short-Time Asymptotic Expansion of the Heat Kernel and Pricing Barrier Options". This research is supported by CARF (Center for Advanced Research in Finance) and the global COE program "The research and training center for new development in mathematics." All the contents expressed in this research are solely those of the authors and do not represent the view of any institutions.

${ }^{\dagger}$ Graduate School of Economics, the University of Tokyo

${ }^{\ddagger}$ Mitsubishi UFJ Trust Investment Technology Institute Co.,Ltd. (MTEC). 
models as special cases; for the local volatility model, we also show how to compute the coefficients in the expansion by using the Lie bracket. Furthermore, we note that the similar method can be applied to a certain class of non-linear parabolic partial differential equations though this paper explicitly deals with the linear PDEs. (Please see Remark 3.1.)

There are many approaches for approximations of heat kernels through certain asymptotic expansions: for instance, there are recent works such as Baudoin (2009), Gatheral-Hsu-Laurence-Ouyang-Wang (2009), Ben Arous-Laurence (2009), Takahashi-Takehara-Toda (2009) and Takahashi-Yamada (2009). On approximation of the solutions to second-order parabolic equations and its applications to option pricing, Cheng et al.(2010,2011) have been developing a new method called Dyson-Taylor Commutator method. Furthermore, Fujii-Takahashi (2011) has developed a new approximation method for the solutions to the nonlinear PDEs associated with the four step scheme for solving forward backward stochastic differential equations (FBSDEs).

The organization of the paper is as follows: After the next section introduces Léandre's Approach, Section 3 derives an integration by parts formula as an extension of a Léandre's theorem and then provides an approximation to the solution of second-order linear parabolic PDEs. Section 4 applies the method developed in the previous section to finance including the valuations of plain-vanilla and barrier options under stochastic volatility environment as well as options' vega. In particular, we provide a new approximate formula and a simple numerical example for down-and-out barrier option prices under a SABR model.

Section 5 derives a short-time asymptotic expansion using integration by parts formula. Section 6 shows examples of the short-time asymptotic expansion under general time-homogeneous local volatility, stochastic volatility model with log-normal local volatility and general local-stochastic volatility models. We also provides numerical examples of the short-time asymptotic expansion under Heston model. Finally, Appendix summarizes the calculation of the second order approximation in Section 6.1.

\section{Malliavin Calculus in Semi-group Theory}

This section summarizes a part of Léandre (2006,2008) which reveals the connections between the semigroup theory and Malliavin calculus. In particular, we introduce Theorem 2.2 below that provides a nice idea for an approximation of the solutions to parabolic PDEs and will be extended in the next section for our purpose.

Consider the following diffusion process on $\mathbf{R}^{n}$ over the $d$-dimensional Wiener space $(\mathcal{W}, H, \mu)$.

$$
\begin{aligned}
d X_{t} & =\sum_{k=1}^{d} V_{k}\left(X_{t}\right) \circ d W_{t}^{k}+V_{0}\left(X_{t}\right) d t, \\
X_{0} & =x_{0} \in \mathbf{R}^{n},
\end{aligned}
$$

where $V_{k}=\left(V_{k}^{1}, \cdots, V_{k}^{n}\right)$ with $V_{k}^{i} \in C_{b}^{\infty}$. Let $A^{i j}(x)=\sum_{k=1}^{d} V_{k}^{i}(x) V_{k}^{j}(x)$ and we assume that a $n \times n$ matrix $A(x)=\left[A^{i j}(x)\right]$ is invertible at any point.

We define $\hat{V}_{k}$ as

$$
\hat{V}_{k}=\sum_{i=1}^{n} V_{k}^{i}(x) \frac{\partial}{\partial x_{i}}, \quad k=0,1, \cdots, d .
$$

and

$$
\mathcal{L}=\frac{1}{2} \sum_{k=1}^{d} \hat{V}_{k}^{2}+\hat{V}_{0}
$$

Associated to the operator $\mathcal{L}$, we consider the following PDE:

$$
\begin{aligned}
\left(\frac{\partial}{\partial t}-\mathcal{L}\right) u(t, x) & =0, \\
u(0, x) & =f(x),
\end{aligned}
$$

where $f \in C_{b}^{2}\left(\mathbf{R}^{n}\right)$. Then, the unique solution $u(t, x)$ has the following form:

$$
u(t, x)=\mathbf{P}_{t} f(x)=E\left[f\left(X_{t}^{x}\right)\right],
$$

where the family of $\left(\mathbf{P}_{t}\right)_{t \geq 0}$ is a Markov semigroup, i.e. $\mathbf{P}_{t+s}=\mathbf{P}_{t} \mathbf{P}_{s} . \mathcal{L}$ is the generator of $\mathbf{P}_{t}$. 
Let $Z$ be the following 1-dimensional process:

$$
\begin{aligned}
d Z_{t} & =Z_{t}\left(\sum_{k=1}^{d} \sum_{i=1}^{n} h_{k}^{i}(t) d W_{t}^{k}\right) \\
Z_{0} & =1 .
\end{aligned}
$$

where $h_{k}^{i} \in L^{2}([0, T])$. Note that $Z_{t}$ is given by

$$
Z_{t}=\exp \left\{\sum_{k=1}^{d} \int_{0}^{t} h_{k}(s) d W_{s}^{k}-\frac{1}{2} \sum_{k=1}^{d} \int_{0}^{t}\left|h_{k}(t)\right|^{2} d s\right\},
$$

where $h_{k}=\sum_{i=1}^{n} h_{k}^{i}$. Define

$$
\hat{h}_{k}=h_{k} \frac{\partial}{\partial z}, \quad k=1, \cdots, d
$$

Let

$$
\tilde{V}_{k}=\hat{V}_{k}+z \hat{h}_{k}, k=1, \cdots, d
$$

Then, let $\tilde{\mathcal{L}}^{h}$ be a generator

$$
\tilde{\mathcal{L}}^{h}=\frac{1}{2} \sum_{k=1}^{d} \tilde{V}_{k}^{2}+\hat{V}_{0}
$$

It generates a time-inhomogenous Markov semigroup $\left(\tilde{\mathbf{P}}_{s, t}^{h}\right)_{\{t \geq s \geq 0\}}$.

Next, for $t \in[0, T](T \in(0, \infty))$ we consider the following diffusion process:

$$
\begin{aligned}
d X_{t}^{h} & =\sum_{k=1}^{d} V_{k}\left(X_{t}\right) \circ d W_{t}^{k}+\sum_{k=1}^{d} h_{k}(t) V_{k}\left(X_{t}\right) d t+V_{0}\left(X_{t}\right) d t \\
X_{0}^{h} & =x_{0} \in \mathbf{R}^{n} .
\end{aligned}
$$

The associated generator is given by

$$
\mathcal{L}^{h}=\mathcal{L}+\sum_{k=1}^{d} h_{k} \hat{V}_{k}
$$

It generates a time-inhomogenous Markov semi-group $\left(\mathbf{P}_{s, t}^{h}\right)_{\{t \geq s \geq 0\}}$.

We write $\mathbf{P}_{0, t}^{h}$ and $\tilde{\mathbf{P}}_{0, t}^{h}$ as $\mathbf{P}_{t}^{h}$ and $\tilde{\mathbf{P}}_{t}^{h}$, respectively.

Theorem 2.1 Consider a $\mathbf{R}$-valued function $\tilde{f}(x, y)=f g(x, y)=f(x) g(y)$ on $\mathbf{R}^{n+1}$ where $f \in C_{b}^{2}\left(\mathbf{R}^{n}\right)$ and $g(y)=y$ for $y \in \mathbf{R}$. Then, the following formula holds.

$$
\mathbf{P}_{t}^{h} f(x)=\tilde{\mathbf{P}}_{t}^{h}[\tilde{f}](x, 1)=\tilde{\mathbf{P}}_{t}^{h}[f g](x, 1) .
$$

Proof 2.1

$$
\begin{aligned}
\tilde{\mathbf{P}}_{t}^{h}[\tilde{f}](x, z) & =E\left[f\left(X_{t}^{x}\right) z \exp \left\{\sum_{k=1}^{d} \int_{0}^{t} h_{k}(s) d W_{s}^{k}-\frac{1}{2} \sum_{k=1}^{d} \int_{0}^{t}\left|h_{k}(t)\right|^{2} d s\right\}\right] \\
& =E\left[f\left(X_{t}^{x}\right) \exp \left\{\sum_{k=1}^{d} \int_{0}^{t} h_{k}(s) d W_{s}^{k}-\frac{1}{2} \sum_{k=1}^{d} \int_{0}^{t}\left|h_{k}(t)\right|^{2} d s\right\}\right] z \\
& =\tilde{\mathbf{P}}_{t}^{h}[\tilde{f}](x, 1) z .
\end{aligned}
$$

Note that

$$
\begin{aligned}
& \left.\left(z h_{k}^{i} V_{k}^{i} \frac{\partial^{2}}{\partial x^{i} \partial z} \tilde{\mathbf{P}}_{t}^{h}[\tilde{f}](x, z)\right)\right|_{z=1}=\left.\left(z h_{k}^{i} V_{k}^{i}\left\{\frac{\partial^{2}}{\partial x^{i} \partial z} \tilde{\mathbf{P}}_{t}^{h}[\tilde{f}](x, 1) z\right\}\right)\right|_{z=1} \\
& =h_{k}^{i} V_{k}^{i} \frac{\partial}{\partial x^{i}} \tilde{\mathbf{P}}_{t}^{h}[\tilde{f}](x, 1) .
\end{aligned}
$$


Then,

$$
\left.\tilde{\mathcal{L}}^{h} \tilde{\mathbf{P}}_{t}^{h}[\tilde{f}](x, z)\right|_{z=1}=\mathcal{L}^{h} \tilde{\mathbf{P}}_{t}^{h}[\tilde{f}](x, 1)
$$

We also have

$$
\left.\left(\frac{\partial}{\partial t}-\tilde{\mathcal{L}}^{h}\right) \tilde{\mathbf{P}}_{t}^{h}[\tilde{f}](x, z)\right|_{z=1}=0
$$

Therefore, $u(t, x)=\tilde{\mathbf{P}}_{t}^{h}[\tilde{f}](x, 1)$ satisfies

$$
\left(\frac{\partial}{\partial t}-\tilde{\mathcal{L}}^{h}\right) u(t, x)=\left(\frac{\partial}{\partial t}-\mathcal{L}^{h}\right) u(t, x)=0 .
$$

On the other hand, $F(t, x)=\mathbf{P}_{t}^{h} f(x)$ satisfies

$$
\left(\frac{\partial}{\partial t}-\mathcal{L}^{h}\right) F(t, x)=0
$$

Then, the result follows from the uniqueness of the solution.

\section{Remark}

$$
\mathbf{P}_{t}^{h} f(x)=\int_{\mathcal{W}} f\left(X_{t}^{h, x}\right) d \mu^{h}(w)=\int_{\mathcal{W}} f\left(X_{t}^{x}\right) Z_{t}^{1} d \mu(w)=\tilde{\mathbf{P}}_{t}^{h}[\tilde{f}](x, 1),
$$

where $\mu^{h}$ is the shifted Wiener measure in the direction of $h \in H$, i.e., $\mu^{h}(w)=\mu(w+h)$.

We consider the following perturbed diffusion process, for $t \in[0, T]$ :

$$
\begin{aligned}
d X_{t}^{(\epsilon)} & =\sum_{k=1}^{d} V_{k}\left(X_{t}^{(\epsilon)}\right) \circ d W_{t}^{k}+\sum_{k=1}^{d} \epsilon h_{k}(t) V_{k}\left(X_{t}^{(\epsilon)}\right) d t+V_{0}\left(X_{t}^{(\epsilon)}\right) d t \\
X_{0}^{(\epsilon)} & =x_{0} \in \mathbf{R}^{n},
\end{aligned}
$$

where $\epsilon \in[0,1]$.

The associated generator is given by

$$
\mathcal{L}^{\epsilon h}=\mathcal{L}+\epsilon \sum_{k=1}^{d} h_{k} \hat{V}_{k}
$$

$\epsilon \in[0,1]$

Let $f \in C_{b}^{2}\left(\mathbf{R}^{n}\right) . u^{\epsilon}(t, x):=\mathbf{P}_{t}^{\epsilon} f(x)=E\left[f\left(X_{t}^{x,(\epsilon)}\right)\right], t \in[0, T], x \in \mathbf{R}^{n}$ is the unique solution to the following PDE:

$$
\begin{aligned}
\left(\frac{\partial}{\partial t}-\mathcal{L}^{\epsilon}\right) u^{\epsilon}(t, x) & =0, \quad t \in(0, T] \\
u^{\epsilon}(0, x) & =f(x) .
\end{aligned}
$$

Note also that $u^{0}(t, x)=\mathbf{P}_{t}^{0} f(x)=\mathbf{P}_{t} f(x)=E\left[f\left(X_{t}^{x}\right)\right], t \in[0, T], x \in \mathbf{R}^{n}$ is the unique solution of the following PDE:

$$
\begin{aligned}
\left(\frac{\partial}{\partial t}-\mathcal{L}^{0}\right) u^{0}(t, x) & =0, \quad t \in(0, T] \\
u^{0}(0, x) & =f(x) .
\end{aligned}
$$

Theorem 2.2 below will present a formula for

$$
u_{1}(t, x):=\mathbf{P}_{t}^{1} f(x):=\left.\frac{\partial}{\partial \epsilon}\right|_{\epsilon=0} \mathbf{P}_{t}^{\epsilon} f(x) .
$$

First, it is easily seen that $u_{1}(t, x)$ is the unique solution to the PDE:

$$
\begin{aligned}
\frac{\partial}{\partial t} u_{1}(t, x)-\mathcal{L}^{0} u_{1}(t, x)-\sum_{k=1}^{d} h_{k} \hat{V}_{k} u^{0}(t, x) & =0 \\
u_{1}(0, x) & =0 .
\end{aligned}
$$


Consider the following 1-dimensional process:

$$
\begin{aligned}
d Q_{t} & =\sum_{k=1}^{d} \sum_{i=1}^{n} h_{k}^{i}(t) d W_{t}^{k} \\
Q_{0} & =0
\end{aligned}
$$

Define $h_{k}=\sum_{i=1}^{n} h_{k}^{i}$ and

$$
\bar{h}_{k}(t)=h_{k} \frac{\partial}{\partial q}, \quad k=0,1, \cdots, d .
$$

Then, define $\bar{V}_{k}$ as

$$
\bar{V}_{k}=\hat{V}_{k}+\bar{h}_{k}(t)
$$

where

$$
\bar{h}_{k}(t)=h_{k} \frac{\partial}{\partial q}, \quad k=0,1, \cdots, d,
$$

and the generator $\overline{\mathcal{L}}^{h}$ as

$$
\overline{\mathcal{L}}^{h}=\frac{1}{2} \sum_{k=1}^{d} \bar{V}_{k}^{2}+\hat{V}_{0}
$$

Then, it generate a time inhomogenous semigroup $\left(\overline{\mathbf{P}}_{s, t}^{h}\right)_{\{t \geq s \geq 0\}}$. We write $\overline{\mathbf{P}}_{0, t}^{h}$ as $\overline{\mathbf{P}}_{t}^{h}$.

Theorem 2.2 Elementary integration by parts formula-Léandre (2006,2008)-

Consider a $\mathbf{R}$-valued function $\tilde{f}(x, y)=f g(x, y)=f(x) g(y)$ on $\mathbf{R}^{n+1}$ where $f \in C_{b}^{2}\left(\mathbf{R}^{n}\right)$ and $g(y)=y$ for $y \in \mathbf{R}$. Then, the following formula holds.

$$
u_{1}(t, x)=\overline{\mathbf{P}}_{t}^{h}[\tilde{f}](x, 0)=\overline{\mathbf{P}}_{t}^{h}[f g](x, 0)=\int_{0}^{t} \mathbf{P}_{t-s}^{0} \sum_{k=1}^{d} \sum_{i=1}^{n} h_{k}^{i}(s) V_{k}^{i} \frac{\partial}{\partial x_{i}}\left[\mathbf{P}_{s}^{0} f\right](x) d s .
$$

Proof 2.2 Consider the following PDE:

$$
\begin{aligned}
& \frac{\partial \bar{f}(t, x, q)}{\partial t}=\overline{\mathcal{L}}^{h} \bar{f}(t, x, q), \\
& \bar{f}(0, x, q)=\tilde{f}(x, q) .
\end{aligned}
$$

Then, the unique solution is given by

$$
\bar{f}(t, x, q)=\overline{\mathbf{P}}_{t}^{h}[\tilde{f}](x, q)=\sum_{k=1}^{d} \sum_{i=1}^{n} E\left[f\left(X_{t}^{(0)}\right)\left(q+\int_{0}^{t} h_{k}^{i}(s) d W_{s}^{k}\right)\right] .
$$

Also, we have the relation:

$$
\begin{aligned}
& \overline{\mathbf{P}}_{t}^{h}[\tilde{f}](x, q) \\
= & \sum_{k=1}^{d} \sum_{i=1}^{n} E\left[f\left(X_{t}^{(0)}\right)\left(q+\int_{0}^{t} h_{k}^{i}(s) d W_{s}^{k}\right)\right] \\
= & \sum_{k=1}^{d} \sum_{i=1}^{n} E\left[f\left(X_{t}^{(0)}\right) \int_{0}^{t} h_{k}^{i}(s) d W_{s}^{k}\right]+E\left[f\left(X_{t}^{(0)}\right)\right] q \\
= & \overline{\mathbf{P}}_{t}^{h}[\tilde{f}](x, 0)+\mathbf{P}_{t}^{0}[f](x) q .
\end{aligned}
$$

Thus, we have

$$
\frac{\partial}{\partial t} \overline{\mathbf{P}}_{t}^{h}[\tilde{f}](x, q)=\overline{\mathcal{L}}^{h} \overline{\mathbf{P}}_{t}^{h}[\tilde{f}](x, q)=\overline{\mathcal{L}}^{h}\left\{\overline{\mathbf{P}}_{t}^{h}[\tilde{f}](x, 0)+\mathbf{P}_{t}[f](x) q\right\}
$$


Note that the function $x \mapsto \overline{\mathbf{P}}_{t}^{h}[\tilde{f}](x, 0)$ does not depend on $q$, then

$$
h_{k}^{i} V_{k}^{i} \frac{\partial^{2}}{\partial x^{i} \partial q} \overline{\mathbf{P}}_{t}^{h} \tilde{f}(x, 0)=0,
$$

and

$$
h_{k}^{i} V_{k}^{i} \frac{\partial^{2}}{\partial x^{i} \partial q} \mathbf{P}_{t} f(x) q=h_{k}^{i} V_{k}^{i} \frac{\partial}{\partial x^{i}} \mathbf{P}_{t} f(x)
$$

then

$$
\frac{\partial}{\partial t} \overline{\mathbf{P}}_{t}^{h} \tilde{f}(x, 0)=\mathcal{L}^{0} \overline{\mathbf{P}}_{t}^{h} \tilde{f}(x, 0)+\sum_{k=1}^{d} h_{k} \hat{V}_{k} \mathbf{P}_{t} f(x),
$$

with starting condition 0 . Therefore, $\overline{\mathbf{P}}_{t}^{h}[\tilde{f}](x, 0)$ satisfies $(2.26)$ with starting condition 0 .

On the other hand, it is easily seen that

$$
\begin{aligned}
& \frac{\partial}{\partial t}\left(\int_{0}^{t} \mathbf{P}_{t-s}^{0} \sum_{k=1}^{d} h_{k} \hat{V}_{k} \mathbf{P}_{s}^{0} f(x) d s\right) \\
= & \mathcal{L}^{0}\left(\int_{0}^{t} \mathbf{P}_{t-s}^{0} \sum_{k=1}^{d} h_{k} \hat{V}_{k} \mathbf{P}_{s}^{0} f(x) d s\right)+\sum_{k=1}^{d} h_{k} \hat{V}_{k} \mathbf{P}_{t}^{0} f(x) .
\end{aligned}
$$

Then, $\left(\int_{0}^{t} \mathbf{P}_{t-s}^{0} \sum_{k=1}^{d} h_{k} \hat{V}_{k} \mathbf{P}_{s}^{0} f(x) d s\right)$ satisfies (2.26) with starting condition 0.

The result follows from the uniqueness of the solution of (2.26).

Remark 2.1 (2.32) corresponds to Theorem 2.2 of Léandre(2006) and Theorem 5 of Léandre(2008).

Remark 2.2 Alternatively, we can derive the formula $u_{1}(t, x)=\overline{\mathbf{P}}_{t}^{h}[\tilde{f}](x, 0)$ in Theorem 2.2 in the following manner. Consider the following $n \times n$ matrix-valued process, for $1 \leq i, j \leq n$,

$$
\begin{aligned}
d U_{j}^{i}(t) & =\sum_{l=1}^{d} \sum_{k=1}^{n} A_{k, l}^{i}(s) U_{j}^{k}(s) \circ d W_{t}^{l}+\sum_{k=1}^{n} B_{k}^{i}(s) U_{j}^{k}(s) d s \\
U_{j}^{i}(0) & =\delta_{j}^{i}
\end{aligned}
$$

where

$$
\begin{aligned}
A_{k, l}^{i}(s) & =\partial_{k} V_{l}^{i}\left(X_{s}^{0}\right), \\
B_{k}^{i}(s) & =\partial_{k} V_{0}^{i}\left(X_{s}^{0}\right),
\end{aligned}
$$

and $\delta_{j}^{i}$ is the Kronecker's delta. Let $D_{s, k}, k=1, \cdots, d$ be the Malliavin derivative acting on the Brownian motion $W_{t}^{k}$. Then, it is well-known that for $s \leq t$,

$$
D_{s, k} X_{i, t}^{(0)}=\sum_{l, j=1}^{n} U_{l}^{i}(t) U^{-1}(s)_{j}^{l} V_{k}^{j}\left(X_{s}^{(0)}\right) .
$$

Hence, we obtain that for $f \in C_{b}^{1}$,

$$
\begin{aligned}
& u_{1}(t, x)=\left.\frac{\partial}{\partial \epsilon}\right|_{\epsilon=0} \mathbf{P}_{t}^{\epsilon h} f(x)=\left.\frac{\partial}{\partial \epsilon}\right|_{\epsilon=0} E\left[f\left(X_{t}^{x,(\epsilon)}\right)\right] \\
= & \sum_{i=1}^{n} E\left[\left.\partial_{i} f\left(X_{t}\right) \frac{\partial}{\partial \epsilon}\right|_{\epsilon=0} X_{i, t}^{(\epsilon)}\right] \\
= & \sum_{i=1}^{n} E\left[\partial_{i} f\left(X_{t}\right) \sum_{l, j=1}^{n} \sum_{k=1}^{d} U_{l}^{i}(t) \int_{0}^{t} U^{-1}(s)_{j}^{l} h_{k}^{i}(s) V_{k}^{j}\left(X_{s}^{(0)}\right) d s\right] \\
= & \sum_{k=1}^{d} \sum_{i=1}^{n} E\left[\int_{0}^{t} \partial_{i} f\left(X_{t}^{(0)}\right) D_{s, k} X_{i, t}^{(0)} h_{k}^{i}(s) d s\right]
\end{aligned}
$$




$$
\begin{aligned}
& =\sum_{k=1}^{d} \sum_{i=1}^{n} E\left[\int_{0}^{t} D_{s, k} f\left(X_{t}^{(0)}\right) h_{k}^{i}(s) d s\right] \\
& =\sum_{k=1}^{d} \sum_{i=1}^{n} E\left[f\left(X_{t}^{(0)}\right) \int_{0}^{t} h_{k}^{i}(s) d W_{s}^{k}\right]=\overline{\mathbf{P}}_{t}^{h}[\tilde{f}](x, 0) .
\end{aligned}
$$

Moreover, under the ellipticity of $\mathcal{L}$, the law of $X_{t}^{(0)}$, has the form $p^{X^{(0)}}(t, x, y) d y$ where $d y$ is the Lebesgue measure and hence, we easily obtain the following.

\section{Corollary 2.1}

$$
\overline{\mathbf{P}}^{h}[\tilde{f}](x, 0)=\overline{\mathbf{P}}^{h}[f g](x, 0)=\int_{\mathbf{R}^{n}} f(y) g^{0}(y) p^{X^{(0)}}(t, x, y) d y
$$

where

$$
g^{0}(y)=E\left[\sum_{k=1}^{d} \sum_{i=1}^{n} \int_{0}^{t} h_{k}^{i}(s) d W_{s}^{k} \mid X_{t}^{(0)}=y\right]
$$

\section{Integration by Parts Formula and Asymptotic Expansion of the Solution to Parabolic PDEs}

In this section, we will extend Léandre's "elementary integration by parts formula" (Theorem 2.2 in the previous section) to Proposition 3.1 below, and present an approximation formula((3.47) in Theorem 3.1) of the solution to a second-order linear parabolic partial differential equation.

Let $X^{(\epsilon)}$ be the unique solution to the following $n$-dimensional perturbed SDE: for $\epsilon \in[0,1]$,

$$
\begin{aligned}
d X_{t}^{(\epsilon)} & =\sum_{k=1}^{d} V_{k}\left(\epsilon, X_{t}^{(\epsilon)}\right) \circ d W_{t}^{k}+V_{0}\left(\epsilon, X_{t}^{(\epsilon)}\right) d t \\
X_{0} & =x \in \mathbf{R}^{n},
\end{aligned}
$$

or

$$
\begin{aligned}
d X_{t}^{(\epsilon)} & =\sum_{k=1}^{d} V_{k}\left(\epsilon, X_{t}^{(\epsilon)}\right) d W_{t}^{k}+\tilde{V}_{0}\left(\epsilon, X_{t}^{(\epsilon)}\right) d t, \\
X_{0} & =x \in \mathbf{R}^{n},
\end{aligned}
$$

where $V_{k}=\left(V_{k}^{1}, \cdots, V_{k}^{n}\right)(k=0,1, \cdots, d)$ have bounded derivatives of any orders in the variables $(\epsilon, x)$ and

$$
\tilde{V}_{0}^{i}(\epsilon, x)=V_{0}^{i}(\epsilon, x)+\frac{1}{2} \sum_{l=1}^{n} \sum_{k=1}^{d} \partial_{l} V_{k}^{i}(\epsilon, x) V_{k}^{l}(\epsilon, x) .
$$

Here, "o" indicates the stochastic differential in the Stratonovich sense.

Also, consider the following $n \times n$ matrix-valued process, $\left\{U_{t}^{(\epsilon)}:\left(U_{j}^{(\epsilon), i}(t)\right), 1 \leq i, j \leq n, 0 \leq t\right\}$,

$$
\begin{aligned}
d U_{j}^{(\epsilon), i}(t) & =\sum_{l=1}^{d} \sum_{k=1}^{n} A_{k, l}^{(\epsilon), i}(s) U_{j}^{(\epsilon), k}(s) \circ d W_{t}^{l}+\sum_{k=1}^{n} B_{k}^{(\epsilon), i}(s) U_{j}^{(\epsilon), k}(s) d s, \\
U_{j}^{(\epsilon), i}(0) & =\delta_{j}^{i},
\end{aligned}
$$

where

$$
\begin{aligned}
& A_{k, l}^{(\epsilon), i}(s)=\partial_{k} V_{l}^{i}\left(\epsilon, X_{s}^{(\epsilon)}\right), \\
& B_{k}^{(\epsilon), i}(s)=\partial_{k} V_{0}^{i}\left(\epsilon, X_{s}^{(\epsilon)}\right)
\end{aligned}
$$

and $\delta_{j}^{i}$ is the Kronecker's delta, that is $U_{0}^{(\epsilon)}=I$ (the identity matrix). Specifically, for $U_{t}^{(0)}$,

$$
\begin{aligned}
& A_{k, l}^{(0), i}(s)=\left.\left[\partial_{k} V_{l}^{i}\left(\epsilon, X_{s}^{(\epsilon)}\right)\right]\right|_{\epsilon=0}, \\
& B_{k}^{(0), i}(s)=\left.\left[\partial_{k} V_{0}^{i}\left(\epsilon, X_{s}^{(\epsilon)}\right)\right]\right|_{\epsilon=0} .
\end{aligned}
$$


Let

$$
X_{t}^{(1, \epsilon)}:=\frac{\partial}{\partial \epsilon} X_{t}^{(\epsilon)}
$$

Then, we have

$$
X_{t}^{(1, \epsilon)}=U_{t}^{(\epsilon)} \int_{0}^{t}\left(U_{s}^{(\epsilon)}\right)^{-1}\left(\sum_{k=1}^{d} \partial_{\epsilon} V_{k}\left(\epsilon, X_{s}^{(\epsilon)}\right) \circ d W_{s}^{k}+\partial_{\epsilon} V_{0}\left(\epsilon, X_{s}^{(\epsilon)}\right) d s\right),
$$

where $\partial_{\epsilon}$ means $\frac{\partial}{\partial \epsilon}$. In particular,

$$
\begin{aligned}
X_{t}^{(1)} & :=X_{t}^{(1,0)}:=\left.\frac{\partial}{\partial \epsilon} X_{t}^{(\epsilon)}\right|_{\epsilon=0} \\
& =U_{t}^{(0)} \int_{0}^{t}\left(U_{s}^{(0)}\right)^{-1}\left(\left.\sum_{k=1}^{d}\left[\partial_{\epsilon} V_{k}\left(\epsilon, X_{s}^{(\epsilon)}\right)\right]\right|_{\epsilon=0} \circ d W_{s}^{k}+\left.\left[\partial_{\epsilon} V_{0}\left(\epsilon, X_{s}^{(\epsilon)}\right)\right]\right|_{\epsilon=0} d s\right) .
\end{aligned}
$$

Next, let $a^{\epsilon}(s)_{k}^{i}, 1 \leq i \leq n, 1 \leq k \leq d$, be the process;

$$
a^{\epsilon}(s)_{k}^{i}=\left(U^{\epsilon}(s)^{-1} V_{k}\left(\epsilon, X_{s}^{(\epsilon)}\right)\right)^{i} .
$$

Then, the reduced Malliavin covariance $V_{*}^{(\epsilon)}(t)=\left\{\left(V_{*}^{\epsilon}(t)\right)^{i j}\right\}_{i, j}$ is expressed as

$$
\left(V_{*}^{\epsilon}(t)\right)^{i j}=\sum_{k=1}^{d} \int_{0}^{t} a^{\epsilon}(s)_{k}^{i} a^{\epsilon}(s)_{k}^{j} d s .
$$

Throughout this section, we assume the following non-degeneracy of the reduced Malliavin covariance:

$$
\text { [A1] } \sup _{\epsilon \in[0,1]} \mathbf{E}\left[\left(\operatorname{det}\left(V_{*}^{(\epsilon)}(t)\right)\right)^{-p}\right]<\infty \text { for } 1<p<\infty .
$$

Then, by Theorem 9.2 in Ikeda-Watanabe (1989), we obtain a smooth density, $y \mapsto p^{\epsilon}(t, x, y)$ associated with (3.1)((3.2)). Moreover, according to Remark 2.2 and Remark 2.3 in Watanabe (1987) as well as Proposition 2.2 in Ikeda-Watanabe (1989), we can see $p^{\epsilon}(t, x, y)$ is smooth in $x$ and $\epsilon$ as well.

We next define $\hat{V}_{k}^{\epsilon}$ as

$$
\hat{V}_{k}^{\epsilon}=\sum_{i=1}^{n} V_{k}^{i}(\epsilon, x) \frac{\partial}{\partial x_{i}}, \quad k=0,1, \cdots, d
$$

and

$$
\mathcal{L}^{\epsilon}=\frac{1}{2} \sum_{k=1}^{d}\left(\hat{V}_{k}^{\epsilon}\right)^{2}+\hat{V}_{0}^{\epsilon}
$$

Next, for $f \in C_{b}\left(\mathbf{R}^{n}\right)$, let

$$
u^{\epsilon}(t, x):=\mathbf{P}_{t}^{\epsilon} f(x):=\mathbf{E}\left[f\left(X_{t}^{(\epsilon)}\right)\right]=\int_{\mathbf{R}^{n}} f(y) p^{\epsilon}(t, x, y) d y .
$$

Then, $u^{\epsilon}(t, x)$ is the solution to the following PDE:

$$
\begin{aligned}
\left(\frac{\partial}{\partial t}-\mathcal{L}^{\epsilon}\right) u^{(\epsilon)}(t, x) & =0, \\
u^{(\epsilon)}(0, x) & =f(x) .
\end{aligned}
$$

Also, let

$$
u^{0}(t, x):=\mathbf{P}_{t}^{0} f(x):=\mathbf{E}\left[f\left(X_{t}^{(0)}\right)\right]=\int_{\mathbf{R}^{n}} f(y) p^{0}(t, x, y) d y,
$$

where $p^{0}(t, x, y)$ is the smooth density for (3.1) with $\epsilon=0$. Then, $u^{0}(t, x)$ is the solution to the following PDE:

$$
\begin{aligned}
\left(\frac{\partial}{\partial t}-\mathcal{L}^{0}\right) u^{0}(t, x) & =0, \\
u^{0}(0, x) & =f(x) .
\end{aligned}
$$




\subsection{Integration by Parts Formula}

In this subsection, we will give the formula for $u^{1}(t, x)=\left.\frac{\partial}{\partial \epsilon} u^{\epsilon}(t, x)\right|_{\epsilon=0}$, and show that $u^{1}(t, x)$ satisfies the following PDE:

$$
\begin{aligned}
\left(\frac{\partial}{\partial t}-\mathcal{L}^{0}\right) u^{1}(t, x) & =\mathcal{L}^{1} u^{0}(t, x) \\
u^{1}(0, x) & =0
\end{aligned}
$$

where

$$
\begin{aligned}
\mathcal{L}^{1}:=\left.\frac{\partial}{\partial \epsilon} \mathcal{L}^{\epsilon}\right|_{\epsilon=0} & =\left.\frac{1}{2} \sum_{i, j=1}^{n} \sum_{k=1}^{d} \frac{\partial}{\partial \epsilon}\left[V_{k}^{i}(\epsilon, x) V_{k}^{j}(\epsilon, x)\right]\right|_{\epsilon=0} \frac{\partial^{2}}{\partial x_{i} \partial x_{j}} \\
& +\left.\sum_{i=1}^{n} \frac{\partial}{\partial \epsilon} V_{0}^{i}(\epsilon, x)\right|_{\epsilon=0} \frac{\partial}{\partial x_{i}} .
\end{aligned}
$$

We obtain the following proposition.

Proposition 3.1 Let $\zeta^{0}(t)$ be the process given by

$$
\zeta^{0}(t)^{l}=\left(V_{*}^{0}(t)^{-1} U^{0}(t)^{-1} X_{t}^{(1)}\right)^{l}, 1 \leq l \leq n .
$$

Then, the following formula holds:

$$
\begin{aligned}
u^{1}(t, x) & =\int_{0}^{t} \mathbf{P}_{t-s}^{0} \mathcal{L}^{1}\left[\mathbf{P}_{s}^{0} f\right](x) d s \\
& =E\left[f\left(X_{t}^{(0)}\right) \sum_{l=1}^{n} \sum_{k=1}^{d}\left\{\zeta^{0}(t)^{l} \int_{0}^{t} a^{0}(s)_{k}^{l} d W_{s}^{k}-\int_{0}^{t} D_{s, k} \zeta^{0}(t)^{l} a^{0}(s)_{k}^{l} d s\right\}\right] \\
& =\int_{\mathbf{R}^{n}} f(y) w(y) p^{0}(t, x, y) d y
\end{aligned}
$$

where $y \mapsto w(y)$ is a smooth function given by

$$
w(y)=E\left[\sum_{l=1}^{n} \sum_{k=1}^{d}\left\{\zeta^{0}(t)^{l} \int_{0}^{t} a^{0}(s)_{k}^{l} d W_{s}^{k}-\int_{0}^{t} D_{s, k} \zeta^{0}(t)^{l} a^{0}(s)_{k}^{l} d s\right\} \mid X_{t}^{(0)}=y\right] .
$$

Proof 3.1 Let $\left\{f_{n}\right\}_{n} \subset C_{b}^{\infty}\left(\mathbf{R}^{n}\right)$ be a sequence such that $f_{n} \rightarrow f$ as $n \rightarrow \infty$. For $E\left[f_{n}\left(X_{t}^{(\epsilon)}\right)\right]$, we can differentiate with respect to $\epsilon$ (and set $\epsilon=0$ ) as follows;

$$
\begin{aligned}
& \left.\frac{\partial}{\partial \epsilon}\right|_{\epsilon=0} E\left[f_{n}\left(X_{t}^{(\epsilon)}\right)\right] \\
= & \sum_{i=1}^{n} E\left[\left.\frac{\partial}{\partial x_{i}} f_{n}\left(X_{t}^{(0)}\right) \frac{\partial}{\partial \epsilon} X_{i t}^{(\epsilon)}\right|_{\epsilon=0}\right] \\
= & E\left[\nabla f_{n}\left(X_{t}^{(0)}\right) \cdot X_{t}^{(1)}\right] \\
= & E\left[\nabla f_{n}\left(X_{t}^{(0)}\right) \cdot U_{t} V_{*}^{0}(t) V_{*}^{0}(t)^{-1} U_{t}^{-1} X_{t}^{(1)}\right] \\
= & \sum_{i=1}^{n} \sum_{m=1}^{n} \sum_{l=1}^{n} E\left[\frac{\partial}{\partial x_{i}} f_{n}\left(X_{t}^{(0)}\right) U(t)_{m}^{i}\left(V_{*}^{0}(t)\right)_{l}^{m}\left(\left(V_{*}^{0}(t)\right)^{-1} U_{t}^{-1} X_{t}^{(1)}\right)^{l}\right. \\
= & \sum_{i=1}^{n} \sum_{m=1}^{n} \sum_{l=1}^{n} E\left[\frac{\partial}{\partial x_{i}} f_{n}\left(X_{t}^{(0)}\right) U(t)_{m}^{i}\left(\sum_{k=1}^{d} \int_{0}^{t}\left(U_{s}^{-1} V_{k}\left(X_{s}^{(0)}\right)\right)^{m}\left(U_{s}^{-1} V_{k}\left(X_{s}^{(0)}\right)\right)^{l} d s\right)\left(\left(V_{*}^{0}(t)\right)^{-1} U_{t}^{-1} X_{t}^{(1)}\right)^{l}\right] \\
= & \sum_{i=1}^{n} \sum_{l=1}^{n} \sum_{k=1}^{d} E\left[\frac{\partial}{\partial x_{i}} f_{n}\left(X_{t}^{(0)}\right) \int_{0}^{t}\left(\sum_{m=1}^{n} U(t)_{m}^{i}\left(U_{s}^{-1} V_{k}\left(X_{s}^{(0)}\right)\right)^{m}\right)\left(U_{s}^{-1} V_{k}\left(X_{s}^{(0)}\right)\right)^{l} d s\left(\left(V_{*}^{0}(t)\right)^{-1} U_{t}^{-1} X_{t}^{(1)}\right)^{l}\right] \\
= & \sum_{i=1}^{n} \sum_{l=1}^{n} \sum_{k=1}^{d} E\left[\frac{\partial}{\partial x_{i}} f_{n}\left(X_{t}^{(0)}\right) \int_{0}^{t}\left(U(t) U_{s}^{-1} V_{k}\left(X_{s}^{(0)}\right)\right)^{i}\left(U_{s}^{-1} V_{k}\left(X_{s}^{(0)}\right)\right)^{l} d s\left(\left(V_{*}^{0}(t)\right)^{-1} U_{t}^{-1} X_{t}^{(1)}\right)^{l}\right]
\end{aligned}
$$




$$
\begin{aligned}
& =\sum_{l=1}^{n} \sum_{k=1}^{d} E\left[\int_{0}^{t} \sum_{i=1}^{n} \frac{\partial}{\partial x_{i}} f_{n}\left(X_{t}^{(0)}\right)\left(U(t) U_{s}^{-1} V_{k}\left(X_{s}^{(0)}\right)\right)^{i}\left(\left(V_{*}^{0}(t)\right)^{-1} U_{t}^{-1} X_{t}^{(1)}\right)^{l}\left(U_{s}^{-1} V_{k}\left(X_{s}^{(0)}\right)\right)^{l} d s\right] \\
& =\sum_{l=1}^{n} \sum_{k=1}^{d} E\left[\int_{0}^{t}\left[D_{s, k} f_{n}\left(X_{t}^{(0)}\right)\right] \zeta^{0}(t)^{l} a^{0}(s)_{k}^{l} d s\right] .
\end{aligned}
$$

In the above equality, $U \equiv U^{0}$, and we used the following relation.

$$
D_{s, k} f_{n}\left(X_{t}^{(0)}\right)=\sum_{i=1}^{n} \frac{\partial}{\partial x_{i}} f_{n}\left(X_{t}^{(0)}\right)\left(U(t) U_{s}^{-1} V_{k}\left(X_{s}^{(0)}\right)\right)^{i} .
$$

For $g=\left(g^{1}, \cdots, g^{n}\right), g^{l}=f_{n}\left(X_{t}^{(0)}\right) \zeta^{0}(t)^{l}$, we have

$$
\sum_{l=1}^{n} \sum_{k=1}^{d} E\left[g^{l} \int_{0}^{t} a^{0}(s)_{k}^{l} d W_{s}^{k}\right]=\sum_{l=1}^{n} \sum_{k=1}^{d} E\left[\int_{0}^{t} D_{t, k} g^{l} a^{0}(s)_{k}^{l} d s\right]
$$

and

$$
D_{s, k} g^{l}=D_{t, k}\left[f_{n}\left(X_{t}^{(0)}\right) \zeta^{0}(t)^{l}\right]=\left[D_{s, k} f_{n}\left(X_{t}^{(0)}\right)\right] \zeta^{0}(t)^{l}+f_{n}\left(X_{t}^{(0)}\right)\left[D_{s, k} \zeta^{0}(t)^{l}\right]
$$

Then

$\sum_{l=1}^{n} \sum_{k=1}^{d} E\left[\int_{0}^{t}\left[D_{s, k} f_{n}\left(X_{t}^{(0)}\right)\right] \zeta^{0}(t)^{l} a^{0}(s)_{k}^{l} d s\right]=\sum_{l=1}^{n} \sum_{k=1}^{d} E\left[f_{n}\left(X_{t}^{(0)}\right)\left\{\zeta^{0}(t)^{l} \int_{0}^{t} a^{0}(s)_{k}^{l} d W_{s}^{k}-\int_{0}^{t} D_{s, k} \zeta^{0}(t)^{l} a^{0}(s)_{k}^{l} d s\right\}\right]$.

Therefore, we obtain the following formula.

$$
\begin{aligned}
\left.\frac{\partial}{\partial \epsilon}\right|_{\epsilon=0} E\left[f_{n}\left(X_{t}^{(\epsilon)}\right)\right] & =\sum_{l=1}^{n} \sum_{k=1}^{d} E\left[f_{n}\left(X_{t}^{(0)}\right)\left\{\zeta^{0}(t)^{l} \int_{0}^{t} a^{0}(s)_{k}^{l} d W_{s}^{k}-\int_{0}^{t} D_{s, k} \zeta^{0}(t)^{l} a^{0}(s)_{k}^{l} d s\right\}\right] \\
& =\int_{\mathbf{R}^{n}} f_{n}(y) w(y) p^{0}(t, x, y) d y
\end{aligned}
$$

where

$$
w(y)=E\left[\sum_{l=1}^{n} \sum_{k=1}^{d}\left\{\zeta^{0}(t)^{l} \int_{0}^{t} a^{0}(s)_{k}^{l} d W_{s}^{k}-\int_{0}^{t} D_{s, k} \zeta^{0}(t)^{l} a^{0}(s)_{k}^{l} d s\right\} \mid X_{t}^{(0)}=y\right] .
$$

The following estimates hold:

$$
\begin{aligned}
& \left|E\left[f\left(X_{t}^{(0)}\right)\right]-E\left[f_{n}\left(X_{t}^{(0)}\right)\right]\right| \leq\left\|f-f_{n}\right\|_{\infty}, \\
& \left|\frac{\partial}{\partial \epsilon}\right|_{\epsilon=0} E\left[f_{n}\left(X_{t}^{(\epsilon)}\right)\right]-E\left[f\left(X_{t}^{(0)}\right) \pi\right] \mid \leq\left\|f-f_{n}\right\|_{\infty}\|\pi\|_{L^{1}},
\end{aligned}
$$

where

$$
\pi=\sum_{l=1}^{n} \sum_{k=1}^{d}\left\{\zeta^{0}(t)^{l} \int_{0}^{t} a^{0}(s)_{k}^{l} d W_{s}^{k}-\int_{0}^{t} D_{s, k} \zeta^{0}(t)^{l} a^{0}(s)_{k}^{l} d s\right\}
$$

Therefore, we obtain as $n \rightarrow \infty$,

$$
\begin{aligned}
u^{1}(t, x) & =\left.\frac{\partial}{\partial \epsilon}\right|_{\epsilon=0} E\left[f\left(X_{t}^{(\epsilon)}\right)\right] \\
& =\sum_{l=1}^{n} \sum_{k=1}^{d} E\left[f\left(X_{t}^{(0)}\right)\left\{\zeta^{0}(t)^{l} \int_{0}^{t} a^{0}(s)_{k}^{l} d W_{s}^{k}-\int_{0}^{t} D_{s, k} \zeta^{0}(t)^{l} a^{0}(s)_{k}^{l} d s\right\}\right] \\
& =\int_{\mathbf{R}^{n}} f(y) w(y) p^{0}(t, x, y) d y .
\end{aligned}
$$


Alternatively, let $\zeta_{s}=\mathbf{P}_{t-s}^{0} \mathbf{P}_{s}^{\epsilon} f(x)$. Then, we have

$$
\mathbf{P}_{t}^{\epsilon} f(x)-\mathbf{P}_{t}^{0} f(x)=\zeta_{t}-\zeta_{0}=\int_{0}^{t} \frac{d}{d s} \zeta_{s} d s=\int_{0}^{t} \mathbf{P}_{t-s}^{0}\left[\mathcal{L}^{\epsilon}-\mathcal{L}^{0}\right] \mathbf{P}_{s}^{\epsilon} f(x) d s .
$$

Hence, using (3.21), we obain

$$
\begin{aligned}
u^{1}(t, x)=\left.\frac{\partial}{\partial \epsilon} u^{\epsilon}(t, x)\right|_{\epsilon=0} & =\lim _{\epsilon \downarrow 0} \frac{1}{\epsilon}\left[\mathbf{P}_{t}^{\epsilon} f(x)-\mathbf{P}_{t}^{0} f(x)\right] \\
& =\lim _{\epsilon \downarrow 0} \frac{1}{\epsilon} \int_{0}^{t} \mathbf{P}_{t-s}^{0}\left[\mathcal{L}^{\epsilon}-\mathcal{L}^{0}\right] \mathbf{P}_{s}^{\epsilon} f(x) d s \\
& =\int_{0}^{t} \mathbf{P}_{t-s}^{0} \mathcal{L}^{1} \mathbf{P}_{s}^{0} f(x) d s .
\end{aligned}
$$

Also, we easily see that

$$
\frac{\partial}{\partial t}\left(\int_{0}^{t} \mathbf{P}_{t-s}^{0} \mathcal{L}^{1} \mathbf{P}_{s}^{0} f(x) d s\right)=\mathcal{L}^{0} \int_{0}^{t} \mathbf{P}_{t-s}^{0} \mathcal{L}^{1} \mathbf{P}_{s}^{0} f(x) d s+\mathcal{L}^{1} \mathbf{P}_{t}^{0} f(x),
$$

and hence, $F(t, x):=\int_{0}^{t} \mathbf{P}_{t-s}^{0} \mathcal{L}^{1} \mathbf{P}_{s}^{0} f(x)$ ds satisfies (3.20) with starting condition 0.

\subsection{Asymptotic Expansion}

Let $H_{(1)}\left(X_{t}^{(\epsilon), x}, \cdot\right): \mathbf{D}_{\infty} \rightarrow \mathbf{D}_{\infty}$ be the divergence operator (Malliavin weight) defined by the Bismut identity (pp.247-248 in Malliavin (1997)):

$$
H_{(1)}\left(X_{t}^{(\epsilon), x}, \Psi_{t}\right)=\sum_{i=1}^{n} \sum_{k=1}^{d}\left[\zeta^{\epsilon}(t)^{i} \int_{0}^{t} a^{\epsilon}(s)_{k}^{i} d W_{s}^{k}-\int_{0}^{t} D_{s, k} \zeta^{\epsilon}(t)^{i} a^{\epsilon}(s)_{k}^{i} d s\right]
$$

where $\Psi_{t}$ is a smooth functional in the Malliavin sense, $\Psi_{t} \in \mathbf{D}_{\infty}\left(\mathbf{R}^{n}\right)$, and

$$
\zeta^{\epsilon}(t)^{i}=\left(V_{*}^{\epsilon}(t)^{-1} U^{\epsilon}(t)^{-1} \Psi_{t}\right)^{i} .
$$

The iterated Malliavin weight $H_{k}$ is recursively defined as follows:

$$
H_{k}\left(X_{t}^{(\epsilon), x}, \Psi_{t}\right)=H_{(1)}\left(X_{t}^{(\epsilon), x}, H_{k-1}\left(X_{t}^{(\epsilon), x}, \Psi_{t}\right)\right),
$$

with

$$
H_{0}\left(X_{t}^{(\epsilon), x}, \Psi_{t}\right) \equiv \Psi_{t} .
$$

The next theorem is our main result in this section.

Theorem 3.1 Consider the following PDE with its initial condition $f \in C_{b}\left(\mathbf{R}^{n}\right)$ :

$$
\begin{aligned}
\left(\frac{\partial}{\partial t}-\mathcal{L}^{\epsilon}\right) u^{\epsilon}(t, x) & =0, \\
u^{\epsilon}(0, x) & =f(x) .
\end{aligned}
$$

Then, its solution

$$
u^{\epsilon}(t, x)=\mathbf{P}_{t}^{\epsilon} f(x)=\mathbf{E}\left[f\left(X_{t}^{(\epsilon)}\right)\right]=\int_{\mathbf{R}^{n}} f(y) p^{\epsilon}(t, x, y) d y,
$$

has an asymptotic expansion in $\mathbf{R}$ :

$$
\mathbf{P}_{t}^{\epsilon} f(x)=\left\{\mathbf{P}_{t}^{0} f(x)+\sum_{j=1}^{N} \epsilon^{j} a_{j}(x)\right\}+O\left(\epsilon^{N+1}\right),
$$


where

$$
\begin{aligned}
a_{j}(x) & =\sum_{k=1}^{j} \sum_{\beta_{1}+\cdots+\beta_{k}=j, \beta_{i} \geq 1} \int_{0}^{t} \int_{0}^{t_{1}} \cdots \int_{0}^{t_{k-1}} \mathbf{P}_{t-t_{1}}^{0} \mathcal{L}^{\beta_{1}} \mathbf{P}_{t_{1}-t_{2}}^{0} \mathcal{L}^{\beta_{2}} \cdots \mathbf{P}_{t_{k-1}-t_{k}}^{0} \mathcal{L}^{\beta_{k}} \mathbf{P}_{t_{k}}^{0} f(x) d t_{k} \cdots d t_{2} d t_{1} \\
& =E\left[f\left(X_{t}^{(0), x}\right) \sum_{k}^{(j)} H_{k}\left(X_{t}^{(0), x}, \prod_{l=1}^{k} X_{\alpha_{l} t}^{0, \beta_{l}}\right)\right] \\
& =\int_{\mathbf{R}^{n}} f(y) w_{j}(y) p^{0}(t, x, y) d y
\end{aligned}
$$

with $\mathcal{L}^{k}:=\left.\frac{1}{k !} \frac{d^{k}}{d \epsilon^{k}} \mathcal{L}^{\epsilon}\right|_{\epsilon=0}, k \in \mathbf{N}$, and

$$
\sum_{k}^{(j)} \equiv \sum_{k=1}^{j} \sum_{\beta_{1}+\cdots+\beta_{k}=j, \beta_{i} \geq 1} \frac{1}{k !}
$$

Here, the so called Malliavin weight $H_{k}\left(X_{t}^{(0), x}, \prod_{l=1}^{k} X_{\alpha_{l}}^{0, \beta_{l}}\right)$ is defined by (3.42) and the push-down of the Malliavin weight $w_{j} \in \mathcal{S}$ is given by

$$
w_{j}(y)=E\left[\sum_{k}^{(j)} H_{k}\left(X_{t}^{(0), x}, \prod_{l=1}^{k} X_{\alpha_{l} t}^{0, \beta_{l}}\right) \mid X_{t}^{(0), x}=y\right],
$$

where $X_{i t}^{(0), k}:=\left.\frac{1}{k !} \frac{d^{k}}{d \epsilon^{k}} X_{i t}^{(\epsilon)}\right|_{\epsilon=0}, k \in \mathbf{N}, i=1, \cdots, n$. Moreover, we obtain a heat kernel expansion in $\mathbf{R}$ :

$$
\begin{aligned}
p^{\epsilon}(t, x, y) & =p^{0}(t, x, y)+\sum_{j=1}^{N} \epsilon^{j} \zeta_{j}(t, x, y)+O\left(\epsilon^{N+1}\right) \\
& =p^{0}(t, x, y)\left(1+\sum_{j=1}^{N} \epsilon^{j} w_{j}(y)\right)+O\left(\epsilon^{N+1}\right),
\end{aligned}
$$

where

$$
\zeta_{j}(t, x, y)=w_{j}(y) p^{0}(t, x, y) .
$$

Proof 3.2 We can recursively apply the integration by parts in Proposition 3.1

$$
\begin{aligned}
u^{j}(t, x) & :=\left.\frac{1}{j !} \frac{\partial^{j}}{\partial \epsilon^{j}} \mathbf{P}_{t}^{\epsilon} f(x)\right|_{\epsilon=0} \\
& =\int_{\mathbf{R}^{n}} f(y) w_{j}(y) p^{0}(t, x, y) d y,
\end{aligned}
$$

where

$$
w_{j}(y)=E\left[\sum_{k}^{(j)} H_{k}\left(X_{t}^{(0), x}, \prod_{l=1}^{k} X_{\alpha_{l} t}^{0, \beta_{l}}\right) \mid X_{t}^{(0), x}=y\right]
$$

Then, we have

$$
\mathbf{P}_{t}^{\epsilon} f(x)=\mathbf{P}_{t}^{0} f(x)+\sum_{j=1}^{N} \epsilon^{j} u^{j}(t, x)+\epsilon^{N+1} \mathbf{R}_{N}(\epsilon) .
$$

where the remainder terms $\mathbf{R}_{N}(\epsilon)$,

$$
\left.\mathbf{R}_{N}(\epsilon)=\int_{0}^{1} \frac{(1-u)^{N}}{N !} E\left[f\left(X_{t}^{(\epsilon u)}\right) \sum_{k}^{(N+1)} H_{k}\left(X_{t}^{(\epsilon u)}, \prod_{l=1}^{k} X_{\alpha_{l} t}^{(\epsilon u), \beta_{l}}\right)\right)\right] d u
$$


which satsfies

$$
E\left[\left|\mathbf{R}_{N}(\epsilon)\right|\right] \leq C(T)\|f\|_{\infty} E\left[\left(\operatorname{det}\left(V_{*}^{(\epsilon)}(t)\right)\right)^{-\gamma}\right]^{\beta}<\infty,
$$

for some $C(T), \gamma, \beta$. (See P.102 in Nualart (2006) for instance.)

Alternatively, we can recursively obtain the following expression of $u^{j}(t, x)$ in the similar way for obtaining (3.40) in the proof of Proposition 3.1:

$u^{j}(t, x)=\sum_{k=1}^{j} \sum_{\beta_{1}+\cdots+\beta_{k}=j, \beta_{i} \geq 1} \int_{0}^{t} \int_{0}^{t_{1}} \cdots \int_{0}^{t_{k-1}} \mathbf{P}_{t-t_{1}}^{0} \mathcal{L}^{\beta_{1}} \mathbf{P}_{t_{1}-t_{2}}^{0} \mathcal{L}^{\beta_{2}} \cdots \mathbf{P}_{t_{k-1}-t_{k}}^{0} \mathcal{L}^{\beta_{k}} \mathbf{P}_{t_{k}}^{0} f(x) d t_{k} \cdots d t_{2} d t_{1}$.

Also, it is easily seen that $u^{j}(t, x)$ satisfies the following PDE:

$$
\left(\frac{\partial}{\partial t}-\mathcal{L}^{0}\right) u^{j}(t, x)=\mathcal{L}^{1} u^{j-1}(t, x)+\cdots+\mathcal{L}^{j} u^{0}(t, x)
$$

Moreover, if we take a sequence $\left\{f_{n}\right\}_{n \in \mathbf{N}}$ such that $f_{n} \in \mathcal{S}, f_{n} \rightarrow \delta_{y}$ as $n \rightarrow \infty$, we have

$$
\mathbf{P}_{t}^{\epsilon} f_{n}(x)={ }_{\mathcal{S}^{\prime}}\left\langle f_{n}, p^{\epsilon}(t, x, \cdot)\right\rangle_{\mathcal{S}} \rightarrow \mathcal{S}^{\prime}\left\langle\delta_{y}, p^{\epsilon}(t, x, \cdot)\right\rangle_{\mathcal{S}}=p^{\epsilon}(t, x, y), n \rightarrow \infty .
$$

Then, the following heat kernel expansion holds :

$$
p^{\epsilon}(t, x, y)=p^{0}(t, x, y)+\sum_{j=1}^{N} \epsilon^{j} \zeta_{j}(t, x, y)+O\left(\epsilon^{N+1}\right)
$$

where

$$
\zeta_{j}(t, x, y)=w_{j}(y) p^{0}(t, x, y)
$$

Therefore, we obtain the results.

Remark 3.1 Let us consider the solution of the PDE:

$$
\left\{\begin{array}{l}
\left(\frac{\partial}{\partial t}+\mathcal{L}^{(\epsilon)}\right) u^{(\epsilon)}(t, x)=0 \\
u^{(\epsilon)}(T, x)=f(x)
\end{array}\right.
$$

Suppose $u^{(\epsilon)}(t, x)$ is expanded by a perturbation method as

$$
u^{(\epsilon)}(t, x)=u^{0}(t, x)+\epsilon u^{1}(t, x)+\epsilon^{2} u^{2}(t, x)+\cdots .
$$

In order to obtain $u^{i}(t, x), i=0,1,2$ for instance, we formally expand the PDE:

$$
\left(\frac{\partial}{\partial t}+\mathcal{L}^{0}+\epsilon \mathcal{L}^{1}+\epsilon^{2} \mathcal{L}^{2}+\cdots\right)\left(u^{0}(t, x)+\epsilon u^{1}(t, x)+\epsilon^{2} u^{2}(t, x)+\cdots\right)=0,
$$

where $\mathcal{L}^{i}=\left.\frac{1}{i !} \frac{\partial^{i} \mathcal{L}^{\epsilon}}{\partial \epsilon^{i}}\right|_{\epsilon=0}$.

Then, $u^{i}(t, x), i=0,1,2$ satisfy the following PDEs:

$$
\begin{aligned}
& \left\{\begin{array}{l}
\left(\frac{\partial}{\partial t}+\mathcal{L}^{0}\right) u^{0}(t, x)=0, \\
u^{0}(T, x)=f(x),
\end{array}\right. \\
& \left\{\begin{array}{l}
\left(\frac{\partial}{\partial t}+\mathcal{L}^{0}\right) u^{1}(t, x)=-\mathcal{L}^{1} u^{0}(t, x), \\
u^{1}(T, x)=0,
\end{array}\right. \\
& \left\{\begin{array}{l}
\left(\frac{\partial}{\partial t}+\mathcal{L}^{0}\right) u^{2}(t, x)=-\left(\mathcal{L}^{1} u^{1}(t, x)+\mathcal{L}^{2} u^{0}(t, x)\right), \\
u^{2}(T, x)=0 .
\end{array}\right.
\end{aligned}
$$

Theorem 3.1 provides a solution to this problem. We note that the same method can be applied, at least formally to a certain class of non-linear parabolic partial differential equations although Theorem 3.1 explicitly deals with the linear ones. A simple example is as follows:

$$
\begin{aligned}
& \left(\partial_{t}+\mathcal{L}^{\epsilon}\right) u^{\epsilon}(t, x)=0,(t<T) ; u^{\epsilon}(T, x)=f(x) \\
& \mathcal{L}^{\epsilon}=\frac{1}{2} \sigma\left(u^{\epsilon}, \partial_{x} u^{\epsilon}\right)^{2} \partial_{x x} \\
& \sigma\left(u^{\epsilon}, \partial_{x} u^{\epsilon}\right)=1+\epsilon\left(u^{\epsilon}+\partial_{x} u^{\epsilon}\right),
\end{aligned}
$$


In this case, we have

$$
\begin{aligned}
\mathcal{L}^{0} & =\frac{1}{2} \partial_{x x}, \\
\mathcal{L}^{1} & =\left(u^{0}(t, x)+\partial_{x} u^{0}(t, x)\right) \partial_{x x}, \\
\mathcal{L}^{2} & =\frac{1}{2}\left\{\left(u^{0}(t, x)+\partial_{x} u^{0}(t, x)\right)^{2}+2\left(u^{1}+\partial_{x} u^{1}\right)\right\} \partial_{x x} .
\end{aligned}
$$

Hence,

$$
\begin{aligned}
& \left(\partial_{t}+\frac{1}{2} \partial_{x x}\right) u^{0}(t, x)=0 ; u^{0}(T, x)=f(x) \\
& \left(\partial_{t}+\frac{1}{2} \partial_{x x}\right) u^{1}(t, x)=-\left(u^{0}(t, x)+\partial_{x} u^{0}(t, x)\right) \partial_{x x} u^{0}(t, x) ; u^{1}(T, x)=0 \\
& \left(\partial_{t}+\frac{1}{2} \partial_{x x}\right) u^{2}(t, x)=-\left(\mathcal{L}^{1} u^{1}(t, x)+\mathcal{L}^{2} u^{0}(t, x)\right) ; \quad u^{2}(T, x)=0 .
\end{aligned}
$$

$u^{0}(t, x)$ is easily solved by (3.73):

$$
u^{0}(t, x)=\int_{-\infty}^{\infty} \frac{1}{\sqrt{2 \pi(T-t)}} e^{\frac{-(z-x)^{2}}{2(T-t)}} f(z) d z .
$$

Then, given $u^{0}(t, x)$, the right hand side of (3.74) is easily computed and so $u^{1}(t, x)$ is solved, too:

$$
\begin{aligned}
u^{1}(t, x) & =\mathbf{E}^{(t, x)}\left[\int_{t}^{T} g\left(s, W_{s}\right) d s\right] \\
& =\int_{t}^{T}\left(\int_{-\infty}^{\infty} \frac{1}{\sqrt{2 \pi(s-t)}} e^{\frac{-(z-x)^{2}}{2(s-t)}} g(s, z) d z\right) d s
\end{aligned}
$$

where

$$
g(s, z)=\left(u^{0}(s, z)+\partial_{x} u^{0}(s, z)\right) \partial_{x x} u^{0}(s, z) .
$$

Recursively, given $u^{0}(t, x)$ and $u^{1}(t, x), u^{2}(t, x)$ is obtained by (3.75).

Moreover, please see Fujii-Takahashi(2011) which has developed a new general approximation method for the solutions to the nonlinear PDEs associated with the four step scheme for solving forward backward stochastic differential equations (FBSDEs).

\section{Perturbations around Closed Form Solutions : Application to Vanilla and Barrier Options}

In this section, we derive approximation formulas for derivatives in stochastic volatility models using the expansion methods developed in Section 3. Hereafter, we use the notation $\int T(x) p(x) d x$ for $T \in \mathcal{S}^{\prime}\left(\mathbf{R}^{n}\right)$ and $p \in \mathcal{S}\left(\mathbf{R}^{n}\right)$ meaning that $\mathcal{S}^{\prime}\left(\mathbf{R}^{n}\right)\langle T, p\rangle_{\mathcal{S}\left(\mathbf{R}^{n}\right)}$.

\subsection{Vega Weight}

Fournié et al. (1999) derive the greeks weights using Malliavin calculus. In this subsection, we obtain the Malliavin weight for the plain-vanilla option's vega(vega weight) by the Bismut identity and show how to derive the analytic approximation of option price using the Vega weight. Let us consider the following asset dynamics.

$$
\begin{aligned}
d S_{t} & =\sigma\left(t, S_{t}\right) d W_{t} . \\
S_{0} & =s_{0},
\end{aligned}
$$

where $\sigma(t, x)>0$. We also consider the perturbed diffusion with $\sigma^{\epsilon}(t, x)=\sigma(t, x)+\epsilon$,

$$
\begin{aligned}
d S_{t}^{\epsilon} & =\sigma^{\epsilon}\left(t, S_{t}^{\epsilon}\right) d W_{t} . \\
S_{0} & =s_{0} .
\end{aligned}
$$


The plain-vanilla (call) option's vega is defined as follows.

$$
v e g a^{L V}=\frac{\partial}{\partial \sigma} E\left[\left(S_{T}-K\right)^{+}\right]=\left.\frac{\partial}{\partial \epsilon} E\left[\left(S_{T}^{\epsilon}-K\right)^{+}\right]\right|_{\epsilon=0}=E\left[\left.\partial\left(S_{T}^{0}-K\right)^{+} S_{T}^{\epsilon}\right|_{\epsilon=0}\right] .
$$

Note that under non-degeneracy of $S_{t},\left(S_{t}-K\right)^{+} \in \mathbf{D}_{-\infty}$ is a Watanabe distribution.

In this case, the option price under $S_{T}^{\epsilon}$ is approximated as follows;

$$
C^{\epsilon}\left(T-t, s_{0}, K\right):=E_{\left(t, s_{0}\right)}\left[\left(S_{T}^{(\epsilon)}-K\right)^{+}\right] \sim \int_{\mathbf{R}}(s-K)^{+} p^{S^{(0)}}\left(T-t, s_{0}, s\right) d s+\epsilon \cdot v_{e g a}{ }^{L V},
$$

where $p^{S^{(0)}}\left(T-t, s_{0}, s\right)$ is the density of $S^{(0)}$.

In the case of $\sigma^{\epsilon}(t, x)=(\sigma+\epsilon) x$,

$$
\begin{aligned}
d S_{t}^{\epsilon} & =(\sigma+\epsilon) S_{t}^{\epsilon} d W_{t} . \\
S_{0}^{\epsilon} & =S_{0}^{0}=s_{0} .
\end{aligned}
$$

The logarithmic process of $S_{t}^{\epsilon}$ is given by,

$$
\begin{aligned}
d X_{t}^{\epsilon} & =(\sigma+\epsilon) d W_{t}-\frac{1}{2}(\sigma+\epsilon)^{2} d t . \\
X_{0}^{\epsilon} & =X_{0}^{0}=x_{0}=\log s_{0} .
\end{aligned}
$$

The associated partial differential equation is given by

$$
\begin{aligned}
\left(\partial_{t}+\mathcal{L}^{\epsilon}\right) u^{(\epsilon)}(t, x) & =0, \\
u^{(\epsilon)}(T, x) & =f\left(e^{x}\right),
\end{aligned}
$$

where $\mathcal{L}^{\epsilon}$ is the generator of $X_{t}^{(\epsilon)}$, i.e.

$$
\mathcal{L}^{\epsilon}=\frac{1}{2}(\sigma+\epsilon)^{2}\left(\frac{\partial^{2}}{\partial x^{2}}-\frac{\partial}{\partial x}\right)
$$

and $f \in C_{b}^{\infty}$.

The Vega is calculated in the following way. Let us consider the process,

$$
\begin{aligned}
d U_{t} & =\sigma U_{t} d W_{t}, \\
U_{0} & =1 .
\end{aligned}
$$

and introduce the process $a(t)$,

$$
a(t)=U_{t}^{-1} \sigma S_{t}
$$

Let $C(T)$ be the reduced Malliavin covariance,

$$
\begin{aligned}
C(T) & :=\int_{t}^{T} a(s)^{2} d s \\
& =\int_{t}^{T}\left(\frac{s_{0}}{S_{s}} \sigma S_{s}\right)^{2} d s \\
& =\left(s_{0} \sigma\right)^{2}(T-t) .
\end{aligned}
$$

Next, we differentiate the underlying asset price at time $T$ with respect to $\epsilon$ at $\epsilon=0$ :

$$
\left.\frac{\partial}{\partial \epsilon} S_{T}^{\epsilon}\right|_{\epsilon=0}=S_{T}\left(W_{T-t}-\sigma(T-t)\right)
$$

Also, we define the process $\xi(t)$ and $\zeta(t)$ as

$$
\begin{aligned}
\xi(T) & :=\left.U_{T}^{-1} \frac{\partial}{\partial \epsilon} S_{T}\right|_{\epsilon=0} \\
& =\frac{s_{0}}{S_{T}} S_{T}\left(W_{T-t}-\sigma(T-t)\right) \\
& =s_{0}\left(W_{T-t}-\sigma T\right) \\
\zeta(T) & :=C(T)^{-1} \xi(T)=\frac{1}{s_{0} \sigma^{2}(T-t)}\left(W_{T-t}-\sigma(T-t)\right)
\end{aligned}
$$


Then, the Malliavin derivative of $\zeta(t)$ is given by

$$
D_{s} \zeta(T)=\frac{1}{s_{0} \sigma^{2}(T-t)} \mathbf{1}_{s \leq T} .
$$

By the integration by parts derived in section $\mathbf{3 . 2}$, the Vega is calculated as follows.

$$
\begin{aligned}
& \left.\frac{\partial}{\partial \epsilon} E_{\left(t, s_{0}\right)}\left[\left(S_{T}^{\epsilon}-K\right)^{+}\right]\right|_{\epsilon=0} \\
= & E_{\left(t, s_{0}\right)}\left[\left(S_{T}^{0}-K\right)^{+}\left\{\zeta(T) \int_{t}^{T} a(s) d W_{s}-\int_{t}^{T} D_{s} \zeta(T) a(s) d s\right\}\right] \\
= & E_{\left(t, s_{0}\right)}\left[\left(S_{T}^{0}-K\right)^{+}\left\{\frac{1}{s_{0} \sigma^{2}(T-t)}\left(W_{T-t}-\sigma(T-t)\right) \int_{t}^{T} s_{0} \sigma d W_{s}-\int_{t}^{T} \frac{1}{s_{0} \sigma^{2}(T-t)} s_{0} \sigma d s\right\}\right] \\
= & \left.E_{\left(t, s_{0}\right)}\left[\left(S_{T}^{0}-K\right)^{+}\left\{\frac{1}{\sigma(T-t)} W_{T-t}^{2}-W_{T-t}-\frac{1}{\sigma}\right\}\right]\right] \\
= & \int_{\mathbf{R}}\left(e^{z}-K\right)^{+} E\left[\frac{1}{\sigma(T-t)} W_{T-t}^{2}-W_{T-t}-\frac{1}{\sigma} \mid X_{T}^{t, x}=z\right] \frac{1}{\sqrt{2 \pi \sigma^{2}(T-t)}} e^{-\frac{\left(z-x-\frac{1}{2} \sigma^{2}(T-t)\right)^{2}}{2 \sigma^{2}(T-t)}} d z \\
= & \int_{\mathbf{R}}\left(e^{z}-K\right)^{+} \vartheta(z) \frac{1}{\sqrt{2 \pi \sigma^{2}(T-t)}} e^{-\frac{\left(z-x-\frac{1}{2} \sigma^{2}(T-t)\right)^{2}}{2 \sigma^{2}(T-t)}} d z,
\end{aligned}
$$

where

$$
\begin{aligned}
\vartheta(z) & :=E\left[\frac{1}{\sigma(T-t)} W_{T-t}^{2}-W_{T-t}-\frac{1}{\sigma} \mid X_{T}^{t, x}=z\right] \\
& =\frac{1}{\sigma^{3}(T-t)}\left(z-x+\frac{1}{2} \sigma^{2}(T-t)\right)^{2}-\frac{1}{\sigma}\left(z-x+\frac{1}{2} \sigma^{2}(T-t)\right)-\frac{1}{\sigma} .
\end{aligned}
$$

Equivalently, we can calculate the Vega by differentiating the semi-group. Recall that $\mathcal{L}^{\epsilon}$ is the generator of $X_{t}^{(\epsilon)}$,

$$
\mathcal{L}^{\epsilon}=\frac{1}{2}(\sigma+\epsilon)^{2}\left(\frac{\partial^{2}}{\partial x^{2}}-\frac{\partial}{\partial x}\right),
$$

We define the differential operators $\mathcal{L}^{0}, \mathcal{L}^{1}$ as follows;

$$
\begin{aligned}
\mathcal{L}^{0} & =\frac{1}{2} \sigma^{2}\left(\frac{\partial^{2}}{\partial x^{2}}-\frac{\partial}{\partial x}\right) \\
\mathcal{L}^{1} & =\left.\frac{\partial}{\partial \epsilon} \mathcal{L}^{\epsilon}\right|_{\epsilon=0}=\sigma\left(\frac{\partial^{2}}{\partial x^{2}}-\frac{\partial}{\partial x}\right) .
\end{aligned}
$$

Using semi-group theory, the Vega is given by

$$
\begin{aligned}
& \left.\frac{\partial}{\partial \epsilon} u^{\epsilon}(t, x)\right|_{\epsilon=0} \\
= & \int_{t}^{T} \mathbf{P}_{s-t}^{0} \mathcal{L}^{1} \mathbf{P}_{T-s}^{0} f\left(e^{x}\right) d s \\
= & \int_{t}^{T} \int_{\mathbf{R}} \int_{\mathbf{R}} p(s-t, x, y) \sigma\left(\frac{\partial^{2}}{\partial y^{2}}-\frac{\partial}{\partial y}\right) p(T-s, y, z) d y f\left(e^{z}\right) d z d s \\
= & \int_{t}^{T}\left(\frac{\partial^{2}}{\partial x^{2}}-\frac{\partial}{\partial x}\right) \int_{\mathbf{R}} \int_{\mathbf{R}} p(s-t, x, y) p(T-s, y, z) d y f\left(e^{z}\right) d z d s \\
= & \left(\int_{t}^{T} d s\right) \sigma\left(\frac{\partial^{2}}{\partial x^{2}}-\frac{\partial}{\partial x}\right) \int_{\mathbf{R}} f\left(e^{z}\right) p(T-t, x, z) d z \\
= & (T-t) \mathcal{L}^{1} \int_{\mathbf{R}} f\left(e^{z}\right) p(T-t, x, z) d z .
\end{aligned}
$$


Note that

$$
\begin{aligned}
& \frac{\partial}{\partial x} p(T-t, x, z) \\
= & \frac{\partial}{\partial x} \frac{1}{\sqrt{2 \pi(T-t)} \sigma} \exp \left\{-\frac{1}{2 \sigma^{2} t}\left(z-x+\frac{1}{2} \sigma^{2}(T-t)\right)^{2}\right\} \\
= & \frac{1}{\sqrt{2 \pi(T-t)} \sigma} \exp \left\{-\frac{1}{2 \sigma^{2}(T-t)}\left(z-x+\frac{1}{2} \sigma^{2}(T-t)\right)^{2}\right\} \frac{1}{\sigma^{2}(T-t)}\left(z-x+\frac{1}{2} \sigma^{2}(T-t)\right),
\end{aligned}
$$

and

$$
\begin{aligned}
& \frac{\partial^{2}}{\partial x^{2}} p(T-t, x, z) \\
= & \frac{\partial^{2}}{\partial x^{2}} \frac{1}{\sqrt{2 \pi(T-t)} \sigma} \exp \left\{-\frac{1}{2 \sigma^{2}(T-t)}\left(z-x+\frac{1}{2} \sigma^{2}(T-t)\right)^{2}\right\} \\
= & \frac{1}{\sqrt{2 \pi(T-t)} \sigma} \exp \left\{-\frac{1}{2 \sigma^{2}(T-t)}\left(z-x+\frac{1}{2} \sigma^{2}(T-t)\right)^{2}\right\}\left\{\left(\frac{1}{\sigma^{2}(T-t)}\left(z-x+\frac{1}{2} \sigma^{2}(T-t)\right)\right)^{2}-\frac{1}{\sigma^{2}(T-t)}\right\} .
\end{aligned}
$$

Then, we obtain

$$
\begin{aligned}
& \left.\frac{\partial}{\partial \epsilon} u^{\epsilon}(t, x)\right|_{\epsilon=0} \\
= & \sigma(T-t) \int_{\mathbf{R}} f\left(e^{z}\right) p(T-t, x, z)\left\{\left(\frac{\left.z-x_{0}+\frac{1}{2} \sigma^{2}(T-t)\right)}{\sigma^{2}(T-t)}\right)^{2}-\frac{1}{\sigma^{2} t}-\frac{\left(z-x_{0}+\frac{1}{2} \sigma^{2}(T-t)\right)}{\sigma^{2}(T-t)}\right\} d z \\
= & \int_{\mathbf{R}} f\left(e^{z}\right) p(T-t, x, z)\left\{\frac{1}{\sigma^{3}(T-t)}\left(x-x_{0}+\frac{1}{2} \sigma^{2}(T-t)\right)^{2}-\frac{1}{\sigma}-\frac{1}{\sigma}\left(x-x_{0}+\frac{1}{2} \sigma^{2}(T-t)\right)\right\} d z .
\end{aligned}
$$

We obtain the Malliavin weight for the Vega,

$$
\vartheta(z)=\frac{1}{\sigma^{3}(T-t)}\left(z-x+\frac{1}{2} \sigma^{2}(T-t)\right)^{2}-\frac{1}{\sigma}-\frac{1}{\sigma}\left(z-x+\frac{1}{2} \sigma^{2}(T-t)\right) .
$$

\subsection{Pricing Options under Stochastic Volatility Model}

This subsection derives an approximate solution of the partial differential equation (PDE) in stochastic volatility model by a perturbation method. We consider the following stochastic volatility model $\left(S_{t}, \sigma_{t}\right)$ :

$$
\begin{aligned}
& d S_{t}^{(\epsilon)}=\sigma_{t}^{(\epsilon)} S_{t}^{(\epsilon)} d W_{1, t}, \\
& d \sigma_{t}^{(\epsilon)}=\epsilon \sigma_{t}^{(\epsilon)}\left(\rho d W_{1, t}+\sqrt{1-\rho^{2}} d W_{2, t}\right),
\end{aligned}
$$

where $\epsilon \in[0,1]$. The purpose of this subsection is to evaluate the European option price which is defined as

$$
C^{S V}\left(T-t, s_{0}, K\right):=E_{\left(t, s_{0}\right)}\left[\left(S_{T}^{(\epsilon)}-K\right)^{+}\right] .
$$

Let $\left(X_{t}^{(\epsilon)}\right)$ denotes the logarithmic process of the underlying asset $\left(S_{t}^{(\epsilon)}\right)$. We also define

$$
\mathbf{P}_{t}^{(\epsilon)} f(x)=E\left[f\left(X_{t}^{(\epsilon)}\right)\right], \quad f \in C_{b}^{\infty},
$$

and a generator

$$
\mathcal{L}^{(\epsilon)}=\frac{1}{2} \sigma^{2} \frac{\partial^{2}}{\partial x^{2}}-\frac{1}{2} \sigma^{2} \frac{\partial}{\partial x}+\epsilon \rho \sigma^{2} \frac{\partial^{2}}{\partial x \partial \sigma}+\epsilon^{2} \frac{1}{2} \sigma^{2} \frac{\partial^{2}}{\partial \sigma^{2}} .
$$

We decompose the generator in three parts, i.e.

$$
\mathcal{L}^{(\epsilon)}=\mathcal{L}^{0}+\epsilon \mathcal{L}_{1}+\epsilon^{2} \mathcal{L}^{2}
$$


where

$$
\begin{aligned}
\mathcal{L}^{0} & =\frac{1}{2} \sigma^{2} \frac{\partial^{2}}{\partial x^{2}}-\frac{1}{2} \sigma^{2} \frac{\partial}{\partial x}, \\
\mathcal{L}^{1} & =\rho \sigma^{2} \frac{\partial^{2}}{\partial x \partial \sigma}, \\
\mathcal{L}^{2} & =\frac{1}{2} \sigma^{2} \frac{\partial^{2}}{\partial \sigma^{2}} .
\end{aligned}
$$

Note that $\mathcal{L}_{0}$ is the (logarithmic) Black-Scholes operator. For $f \in C_{b}^{\infty}$, the solution $u^{(\epsilon)}(t, x)=\mathbf{P}_{T-t}^{(\epsilon)} f\left(e^{x}\right)$ of the PDE,

$$
\left\{\begin{array}{l}
\left(\frac{\partial}{\partial t}+\mathcal{L}^{(\epsilon)}\right) u^{(\epsilon)}(t, x)=0 \\
u^{(\epsilon)}(T, x)=f\left(e^{x}\right)
\end{array}\right.
$$

Let $\left(U_{t}\right)$ be the first variation process defined by $U_{t}:=\frac{\partial}{\partial s_{0}} S_{t}^{(0)}$, i.e.

$$
\begin{aligned}
d U_{t} & =U_{t} \sigma_{t}^{(0)} d W_{1, t}, \\
U_{t} & =1,
\end{aligned}
$$

and $\mathcal{C}(T)$ be the reduced Malliavin (co)variance of $S_{t}^{(\epsilon)}$ at $\epsilon=0$, i.e.,

$$
\mathcal{C}(T)=\int_{t}^{T} a(s)^{2} d s
$$

where

$$
a(t)=\left(U_{t}\right)^{-1} \sigma_{t}^{(0)} S_{t}^{(0)} .
$$

We introduce the expressions;

$$
\begin{aligned}
S_{k T} & =\left.\frac{\partial^{k}}{\partial \epsilon^{k}} S_{T}^{(\epsilon)}\right|_{\epsilon=0}, \\
\xi^{\beta_{1}, \cdots, \beta_{k}}(T) & =\left(U_{T}\right)^{-1} \prod_{i=1}^{k} S_{\beta_{i} T}, \\
\zeta^{\beta_{1}, \cdots, \beta_{k}}(T) & =\mathcal{C}(T)^{-1} \xi^{\beta_{1}, \cdots, \beta_{k}}(T),
\end{aligned}
$$

where $\beta_{l} \geq 1$ satisfy

$$
\sum_{l=1}^{k} \beta_{l}=j, \quad j \in \mathbf{N}, \quad 1 \leq k \leq j .
$$

Theorem 4.1 For $f \in C_{b}^{\infty}$, we have an asymptotic expansion of the solution to PDE,

$$
\begin{aligned}
& \mathbf{P}_{T-t}^{(\epsilon)} f\left(e^{x}\right) \\
= & \mathbf{P}_{T-t}^{(0)} f\left(e^{x}\right)+\sum_{j=1}^{N} \epsilon^{j} \sum^{j} \int_{t}^{T} \int_{t_{1}}^{T} \cdots \int_{t_{k}}^{T} \mathbf{P}_{t_{k_{j}}-t}^{(0)} \mathcal{L}^{\beta_{k_{j}}} \cdots \mathbf{P}_{t_{1}-t_{2}}^{(0)} \mathcal{L}^{\beta_{1}} \mathbf{P}_{T-t_{1}}^{(0)} f\left(e^{x}\right) d t_{k_{j}} \cdots d t_{2} d t_{1}+O\left(\epsilon^{N+1}\right) \\
= & \mathbf{P}_{T-t}^{(0)} f\left(e^{x}\right)+\sum_{j=1}^{N} \epsilon^{j} \int_{\mathbf{R}} f\left(e^{y}\right) w_{j}(t, T, x, y) p^{X_{T-t}^{(0)}}(T-t, x, y) d y+O\left(\epsilon^{N+1}\right),
\end{aligned}
$$

where

$$
\begin{aligned}
\sum^{j} & \equiv \sum_{\beta_{1}+\cdots+\beta_{k_{j}}=j, \beta_{i}=1 o r 2, k_{j} \geq 1}, \\
w_{j}(t, T, x, y) & =\frac{1}{j !} E\left[\vartheta_{(j)}^{(0)} \mid X_{T}^{(0), t, x}=y\right]
\end{aligned},
$$




$$
\begin{aligned}
\vartheta_{(j)}^{(0)} & =\sum_{k=1}^{j} \sum_{\beta_{1}+\cdots+\beta_{k}=j, \beta_{i} \geq 1} \vartheta_{k}\left(\zeta^{\beta_{1}, \cdots, \beta_{k}}(T)\right), \\
\vartheta_{1}\left(\zeta^{\beta_{1}, \cdots, \beta_{k}}(T)\right) & =\zeta^{\beta_{1}, \cdots, \beta_{k}}(T) \int_{t}^{T} a(s) d W_{1, s}-\int_{t}^{T}\left[D_{s, 1} \zeta^{\beta_{1}, \cdots, \beta_{k}}(T)\right] a(s) d s, \\
\vartheta_{k}\left(\zeta^{\beta_{1}, \cdots, \beta_{k}}(T)\right) & =\vartheta_{1} \circ \vartheta_{k-1}\left(\zeta^{\beta_{1}, \cdots, \beta_{k}}(T)\right),
\end{aligned}
$$

and $\mathbf{P}^{(0)}$ is the Black-Scholes semigroup with the generator $\mathcal{L}^{0}$.

Proof 4.1 Under the ellipticity of $\mathcal{L}^{\epsilon}$ and $\sigma_{0}=\sigma_{0}^{(0)}>0$, the solution associated with the stochastic volatility model, $\mathbf{P}_{T-t}^{(\epsilon)} f\left(e^{x}\right)$ has an asymptotic expansion around $\epsilon=0$ and the expansion coefficients are obtained by the following way. The limiting $\left(\epsilon^{0}\right.$-order) term, $\mathbf{P}_{T-t}^{(0)}$ is the (logarithmic) Black-Scholes semi-group with the generator $\mathcal{L}^{0}$. The coefficients of the asymptotic expansion of the solution to PDE are calculated as follows;

$$
\begin{aligned}
& \mathbf{P}_{T-t}^{(\epsilon)} f\left(e^{x}\right)=\mathbf{P}_{T-t}^{(0)} f\left(e^{x}\right)+\epsilon \int_{t}^{T} \mathbf{P}_{t_{1}-t}^{(0)} \mathcal{L}^{1} \mathbf{P}_{T-t_{1}}^{(0)} f\left(e^{x}\right) d t_{1}+\cdots \\
+ & \epsilon^{j} \sum^{j} \int_{t}^{T} \int_{t_{1}}^{T} \cdots \int_{t_{k_{j}-1}}^{T} \mathbf{P}_{t_{k_{j}}-t}^{(0)} \mathcal{L}^{\beta_{k_{j}}} \cdots \mathcal{L}^{\beta_{2}} \mathbf{P}_{t_{1}-t_{2}}^{(0)} \mathcal{L}^{\beta_{1}} \mathbf{P}_{T-t_{1}}^{(0)} f\left(e^{x}\right) d t_{k_{j}} \cdots d t_{2} d t_{1} \\
+ & O\left(\epsilon^{j+1}\right),
\end{aligned}
$$

For the coefficient of $\epsilon^{j}$, note that

$$
\begin{aligned}
& \frac{\partial}{\partial t} \sum^{j} \int_{t}^{T} \int_{t_{1}}^{T} \cdots \int_{t_{k_{j-1}}}^{T} \mathbf{P}_{t_{k_{j}}-t}^{(0)} \mathcal{L}^{\beta_{k_{j}}} \cdots \mathcal{L}^{\beta_{2}} \mathbf{P}_{t_{1}-t_{2}}^{(0)} \mathcal{L}^{\beta_{1}} \mathbf{P}_{T-t_{1}}^{(0)} f\left(e^{x}\right) d t_{k_{j}} \cdots d t_{2} d t_{1} \\
& =-\mathcal{L}^{0} u^{j}(t, x)-\mathcal{L}^{2} u^{j-2}(t, x)-\mathcal{L}^{1} u^{j-1}(t, x) .
\end{aligned}
$$

Then, the coefficient of $\epsilon^{j}, \sum^{j} \int_{t}^{T} \int_{t_{1}}^{T} \cdots \int_{t_{k-1}}^{T} \mathbf{P}_{t_{k_{j}}-t}^{(0)} \mathcal{L}^{\beta_{k_{j}}} \cdots \mathcal{L}^{\beta_{2}} \mathbf{P}_{t_{1}-t_{2}}^{(0)} \mathcal{L}^{\beta_{1}} \mathbf{P}_{T-t_{1}}^{(0)} f\left(e^{x}\right) d t_{k_{j}} \cdots d t_{2} d t_{1}$ satisfies

$$
\begin{aligned}
\left(\frac{\partial}{\partial t}+\mathcal{L}^{0}\right) u^{j}(t, x) & =-\mathcal{L}^{1} u^{j-1}(t, x)-\mathcal{L}^{2} u^{j-2}(t, x), \\
u^{j}(T, x) & =0 .
\end{aligned}
$$

On the other hand,

$$
\begin{aligned}
\left.\frac{\partial}{\partial \epsilon} \mathbf{P}_{T-t}^{(\epsilon)} f\left(e^{x}\right)\right|_{\epsilon=0} & =E_{\left(t, s_{0}\right)}\left[f^{\prime}\left(S_{T}^{(0)}\right) S_{1 T}\right] \\
& =E_{\left(t, s_{0}\right)}\left[f^{\prime}\left(S_{T}^{(0)}\right) U_{T} \mathcal{C}(T) \zeta^{1}(T)\right] .
\end{aligned}
$$

By the chain rule of Malliavin calculus, $u \in[0, t]$,

$$
\begin{aligned}
D_{u, 1} f\left(S_{T}^{(0)}\right) & =f^{\prime}\left(S_{T}^{(0)}\right) U_{T} U_{u}^{-1} \sigma_{u}^{(0)} S_{u}^{(0)}, \\
D_{u, 2} f\left(S_{T}^{(0)}\right) & =0 .
\end{aligned}
$$

Then, we obtain

$$
\left.\frac{\partial}{\partial \epsilon} \mathbf{P}_{T-t}^{(\epsilon)} f\left(e^{x}\right)\right|_{\epsilon=0}=E_{\left(t, s_{0}\right)}\left[\int_{t}^{T}\left\{\left[D_{s, 1} f\left(S_{T}^{(0)}\right)\right] \zeta^{1}(t)\right\} a_{1}(s) d s\right] .
$$

Note that, for $u \leq T$,

$$
D_{u, 1}\left\{f\left(S_{T}^{(0)}\right) \zeta^{1}(T)\right\}=\left[D_{u, 1} f\left(S_{T}^{(0)}\right)\right] \zeta^{1}(T)+f\left(S_{T}^{(0)}\right)\left[D_{u, 1} \zeta^{1}(T)\right] .
$$

By the integration by parts formula,

$$
\begin{aligned}
& E\left[\int_{t}^{T}\left\{\left[D_{u, 1} f\left(S_{T}^{(0)}\right)\right] \zeta^{1}(T)\right\} a(u) d u\right] \\
= & E\left[f\left(e^{X_{T}^{(0)}}\right)\left\{\zeta^{1}(T) \int_{t}^{T} a(u) d W_{1, u}-\int_{t}^{T}\left[D_{u, 1} \zeta^{1}(T)\right] a(u) d u\right\}\right],
\end{aligned}
$$


and we obtain

$$
\begin{aligned}
& \left.\frac{\partial}{\partial \epsilon} \mathbf{P}_{T-t}^{(\epsilon)} f\left(e^{x}\right)\right|_{\epsilon=0} \\
= & \int_{\mathbf{R}} f\left(e^{y}\right) E\left[\zeta^{1}(T) \int_{t}^{T} a(u) d W_{1, u}-\int_{t}^{T}\left[D_{u, 1} \zeta^{1}(T)\right] a(u) d u \mid X_{T}^{(0)}=y\right] p^{X_{t}^{(0)}}(t, x, y) d y .
\end{aligned}
$$

The higher order approximation terms of the expansion is given as follows;

$$
\left.\frac{1}{i !} \frac{\partial^{i}}{\partial \epsilon^{i}} \mathbf{P}_{T-t}^{(\epsilon)} f\left(e^{x}\right)\right|_{\epsilon=0}=\frac{1}{i !} \int_{\mathbf{R}} f\left(e^{y}\right) E\left[\vartheta_{(i)}^{(0)} \mid X_{T}^{(0), t, x}=y\right] p^{X_{T}^{(0), t, x}}(T-t, x, y) d y,
$$

where $\vartheta_{(i)}^{(0)} \in \mathbf{D}_{\infty}$. Then, we obtain an asymptotic expansion formula of the solution to PDE of the stochastic volatility model around the Black-Scholes solution,

$$
\mathbf{P}_{T-t}^{(\epsilon)} f\left(e^{x}\right)=\mathbf{P}_{T-t}^{(0)} f\left(e^{x}\right)+\sum_{j=1}^{N} \frac{\epsilon^{j}}{j !} \int_{\mathbf{R}} f\left(e^{y}\right) E\left[\vartheta_{(j)}^{(0)} \mid X_{T}^{(0), t, x}=y\right] p^{X_{T}^{(0), t, x}}(T-t, x, y) d y+O\left(\epsilon^{N+1}\right) .
$$

In Corollary 4.1 below, we specifically derive the first order approximation formula of European option under the stochastic volatility model.

Corollary 4.1 The following approximation formula holds.

$$
C^{S V}\left(T-t, e^{x}, K\right)=C^{B S}\left(T-t, e^{x}, K\right)+\epsilon C_{1}\left(T-t, e^{x}, K\right)+O\left(\epsilon^{2}\right),
$$

where $C^{B S}(T-t, z, K)$ denotes the Black-Scholes European option price (with time-to-maturity $T-t$, spot price $z$ and strike price $K$ ) and

$$
\begin{aligned}
C_{1}\left(T-t, e^{x}\right) & =\int_{\mathbf{R}}\left(e^{z}-K\right)^{+} w_{1}(t, T, x, z) p^{X^{(0)}}(T-t, x, z) d y \\
& =\frac{(T-t)}{2} \rho \sigma_{0} e^{x} n\left(d_{1}\right)\left(-d_{2}\right),
\end{aligned}
$$

with

$$
\begin{aligned}
& w_{1}(t, T, x, z) \\
= & \rho \sigma^{3} \frac{(T-t)^{2}}{2}\left(\frac{\left(z-x+\frac{1}{2} \sigma^{2}(T-t)\right)^{3}}{\left(\sigma^{2}(T-t)\right)^{3}}-\frac{3\left(z-x+\frac{1}{2} \sigma^{2}(T-t)\right)}{\left(\sigma^{2}(T-t)\right)^{2}}-\frac{\left(z-x+\frac{1}{2} \sigma^{2}(T-t)\right)^{2}}{\left(\sigma^{2}(T-t)\right)^{2}}-\frac{1}{\sigma^{2}(T-t)}\right), \\
& d_{1}=\frac{\log \left(e^{x} / K\right)+\sigma_{0}^{2}(T-t) / 2}{\sigma_{0} \sqrt{T-t}}, \\
& d_{2}=d_{1}-\sigma \sqrt{T-t} \\
& n\left(d_{1}\right)=\frac{1}{\sqrt{2 \pi}} \exp \left(\frac{-d_{1}^{2}}{2}\right) .
\end{aligned}
$$

\section{Proof}

By Theorem 4.1 ,

$$
\begin{aligned}
& \left.\frac{\partial}{\partial \epsilon} \mathbf{P}_{T-t}^{(\epsilon)} f\left(e^{x}\right)\right|_{\epsilon=0} \\
= & \int_{t}^{T} \mathbf{P}_{s-t}^{0} \mathcal{L}^{1} \mathbf{P}_{T-s}^{0} f\left(e^{x}\right) d s \\
= & \int_{\mathbf{R}} f\left(e^{z}\right) E\left[\zeta^{1}(T) \int_{t}^{T} a(u) d W_{1, u}-\int_{t}^{T}\left[D_{u, 1} \zeta^{1}(T)\right] a(u) d u \mid X_{T}^{(0) t, x}=y\right] p^{X^{(0)}}(T-t, x, z) d y .
\end{aligned}
$$

The conditional expectation above is evaluated as follows;

$$
\begin{aligned}
& E\left[\zeta^{1}(T) \int_{t}^{T} a(u) d W_{1, u}-\int_{t}^{T}\left[D_{u, 1} \zeta^{1}(T)\right] a(u) d u \mid X_{T}^{(0) t, x}=y\right] \\
= & \rho \sigma^{3} \frac{(T-t)^{2}}{2}\left(\frac{\left(z-x+\frac{1}{2} \sigma^{2}(T-t)\right)^{3}}{\left(\sigma^{2}(T-t)\right)^{3}}-\frac{3\left(z-x+\frac{1}{2} \sigma^{2}(T-t)\right)}{\left(\sigma^{2}(T-t)\right)^{2}}-\frac{\left(z-x+\frac{1}{2} \sigma^{2}(T-t)\right)^{2}}{\left(\sigma^{2}(T-t)\right)^{2}}-\frac{1}{\sigma^{2}(T-t)}\right) .
\end{aligned}
$$


Note that

$$
\mathcal{L}^{1} \mathbf{P}_{T-t}^{(0)} f\left(e^{x}\right)=\rho \sigma_{0}^{3}(T-t)\left(\frac{\partial^{3}}{\partial x^{3}}-\frac{\partial^{3}}{\partial x^{3}}\right) \mathbf{P}_{T-t}^{(0)} f\left(e^{x}\right)
$$

Therefore,

$$
\begin{aligned}
& \int_{t}^{T} \mathbf{P}_{s-t}^{0} \mathcal{L}^{1} \mathbf{P}_{T-s}^{0} f\left(e^{x}\right) d s \\
= & \rho \sigma_{0}^{2} \int_{t}^{T} \int_{\mathbf{R}}\left(\frac{\partial^{2}}{\partial y \partial \sigma_{0}} \mathbf{P}_{T-s}^{0} f\left(e^{y}\right)\right) p^{X^{(0)}}(s-t, x, y) d y d s \\
= & \rho \sigma_{0}^{3} \int_{t}^{T} \int_{-\infty}^{\infty}(T-s)\left(\left(\frac{\partial^{3}}{\partial y^{3}}-\frac{\partial^{2}}{\partial y^{2}}\right) \mathbf{P}_{T-s}^{0} f\left(e^{y}\right)\right) p^{X^{(0)}}(s-t, x, y) d y d s, \\
= & \rho \sigma_{0}^{3} \int_{t}^{T}(T-s) \int_{\mathbf{R}} \int_{\mathbf{R}} p^{X^{(0)}}(s-t, x, y)\left(\frac{\partial^{3}}{\partial y^{3}}-\frac{\partial^{2}}{\partial y^{2}}\right) p^{X^{(0)}}(T-s, y, z) d y f\left(e^{z}\right) d z d s \\
= & \rho \sigma_{0}^{3} \int_{t}^{T}(T-s)\left(\frac{\partial^{3}}{\partial x^{3}}-\frac{\partial^{2}}{\partial x^{2}}\right) \int_{\mathbf{R}} \int_{\mathbf{R}} p^{X^{(0)}}(s-t, x, y) p^{X^{(0)}}(T-s, y, z) d y f\left(e^{z}\right) d z d s \\
= & \rho \sigma_{0}^{3}\left(\int_{t}^{T}(T-s) d s\right)\left(\frac{\partial^{3}}{\partial x^{3}}-\frac{\partial^{2}}{\partial x^{2}}\right) \int_{\mathbf{R}} p^{X^{(0)}}(T-t, x, z) f\left(e^{z}\right) d z \\
= & \rho \sigma_{0}^{3} \frac{(T-t)^{2}}{2}\left(\frac{\partial^{3}}{\partial x^{3}}-\frac{\partial^{2}}{\partial x^{2}}\right) \mathbf{P}_{T-t}^{0} f\left(e^{x}\right) .
\end{aligned}
$$

Take a sequence $\left\{f_{n}\right\}_{n}$ such that $f_{n} \in \mathcal{S}, f_{n} \rightarrow(\cdot-K)^{+}$in $\mathcal{S}^{\prime}(n \rightarrow \infty)$, we have

$$
\begin{aligned}
\int_{\mathbf{R}} f_{n}\left(e^{z}\right) p^{X^{(\epsilon)}}(T-t, x, z) d z & \rightarrow C^{S V}(T-t, x, K), \\
\int_{\mathbf{R}} f_{n}\left(e^{z}\right) p^{X^{(0)}}(T-t, x, z) d z & \rightarrow C^{B S}\left(T-t, e^{x}, K\right), \\
\int_{\mathbf{R}} f_{n}\left(e^{z}\right) w_{1}(t, T, x, z) p^{X^{(0)}}(T-t, x, z) d z & \rightarrow \mathcal{S}^{\prime}\left\langle(e \cdot K)^{+}, w_{1}(t, T, x, \cdot) p^{X^{(0)}}(T-t, x, \cdot)\right\rangle_{\mathcal{S}},
\end{aligned}
$$

as $n \rightarrow \infty$. Then, we obtain

$$
\begin{aligned}
& C^{S V}\left(T-t, e^{x}, K\right) \\
= & C^{B S}\left(T-t, e^{x}, K\right)+\epsilon \rho \sigma_{0}^{3} \frac{(T-t)^{2}}{2}\left(\frac{\partial^{3}}{\partial x^{3}}-\frac{\partial^{2}}{\partial x^{2}}\right) C^{B S}(T-t, x, K)+O\left(\epsilon^{2}\right) \\
= & C^{B S}\left(T-t, e^{x}, K\right)+\epsilon \frac{(T-t)}{2} \rho \sigma_{0} e^{x} n\left(d_{1 t}\right)\left(-d_{2 t}\right)+O\left(\epsilon^{2}\right) \\
= & C^{B S}\left(T-t, e^{x}, K\right)+\epsilon \int_{\mathbf{R}}\left(e^{z}-K\right)^{+} w_{1}(t, T, x, z) p^{X^{(0)}}(T-t, x, z) d z+O\left(\epsilon^{2}\right) .
\end{aligned}
$$

\subsection{Barrier Option under Stochastic Volatility Model}

In this subsection, we deal with a barrier option of which value is given under a risk-neutral probability measure as

$$
C_{B}\left(T-t, s_{0}, K\right)=E_{\left(t, s_{0}\right)}\left[f\left(S_{T}^{(\epsilon)}\right) \mathbf{1}_{\left\{\tau^{\epsilon}>T\right\}}\right],
$$

where $f(\cdot)$ stands for a dsicounted payoff function, $S_{T}^{(\epsilon)}$ denotes an underlying asset value at $T$ and $\tau^{\epsilon}$ is the first hitting time of the region $\{x: x \geq B\}$, i.e. $\tau^{\epsilon}=\inf \left\{t ; S_{t}^{(\epsilon)}=B\right\}$. We cannot directly apply the Malliavin calculus to the above because Malliavin derivative $D_{t} \tau^{\epsilon}$ does not exist (see Remark 2.2 in Fournié et al. (2001)). However, according to the PDE method developed in Section 4.2 we can obtain the expansion coefficients by differentiating (4.43) with respect to $\epsilon$, that is,

$$
C_{1}(T-t, s, K)=\left.\frac{\partial}{\partial \epsilon}\right|_{\epsilon=0} E_{\left(t, s_{0}\right)}\left[f\left(S_{T}^{(\epsilon)}\right) \mathbf{1}_{\left\{\tau^{\epsilon}>T\right\}}\right],
$$

(4.44) is regarded as an extension of the plain-vanilla option case.

This section shows how to derive a new formula for the down-and-out call option prices; the same method can be applied to the other types of barrier options such as the up-and-out option. 


\subsection{1 $\lambda$-SABR with $\beta=1$}

We will derive an approximation formula for the down-and-out call option price under $\lambda$-SABR model with $\beta=1$. That is, suppose that the risk-neutral dynamics of the underlying asset price is given as the following: for $\epsilon \in(0,1]$,

$$
\begin{aligned}
& d S_{t}^{\epsilon}=\epsilon \alpha S_{t}^{\epsilon} d t+\sigma_{t}^{\epsilon} S_{t}^{\epsilon} d W_{t}^{1} ; S_{0}=s_{0}, \\
& d \sigma_{t}^{\epsilon}=\epsilon \lambda\left(\theta-\sigma_{t}^{\epsilon}\right) d t+\epsilon \sigma_{t}^{\epsilon}\left(\rho d W_{t}^{1}+\sqrt{1-\rho^{2}} d W_{t}^{2}\right) ; \sigma_{0}^{\epsilon}=\sigma_{0},
\end{aligned}
$$

where $\alpha$ is a constant, and $\lambda$ and $\theta$ are positive constants. Let $X=\log S$ and then,

$$
\begin{aligned}
d X_{t}^{\epsilon} & =\left(\epsilon \alpha-\frac{\left(\sigma_{t}^{\epsilon}\right)^{2}}{2}\right) d t+\sigma_{t}^{\epsilon} d W_{t}^{1} ; X_{0}^{\epsilon}=x \\
d \sigma_{t}^{\epsilon} & =\epsilon \lambda\left(\theta-\sigma_{t}^{\epsilon}\right) d t+\epsilon \sigma_{t}^{\epsilon}\left(\rho d W_{t}^{1}+\sqrt{1-\rho^{2}} d W_{t}^{2}\right) ; \sigma_{0}^{\epsilon}=\sigma_{0} .
\end{aligned}
$$

The asscociated generator, $\mathcal{L}^{\epsilon}$ and the partial differential equation for the down-and-out call option price with barrier price $B\left(<S_{0}\right)$, maturity $T$ and strike $K$ are obtained as follows:

$$
\begin{gathered}
\mathcal{L}^{\epsilon}=\frac{\left(\sigma_{t}^{\epsilon}\right)^{2}}{2} \partial_{x x}+\epsilon \rho\left(\sigma_{t}^{\epsilon}\right)^{2} \partial_{x \sigma}+\epsilon^{2} \frac{\left(\sigma_{t}^{\epsilon}\right)^{2}}{2} \partial_{\sigma \sigma}+\left(\epsilon \alpha-\frac{\left(\sigma_{t}^{\epsilon}\right)^{2}}{2}\right) \partial_{x}+\epsilon \lambda\left(\theta-\sigma_{t}^{\epsilon}\right) \partial_{\sigma} \\
\left(\partial_{t}+\mathcal{L}^{\epsilon}\right) u^{\epsilon}=0 ;(0 \leq t<T, x>b \equiv \log B) \\
u^{\epsilon}(T, x)=f\left(e^{x}\right)=\left(e^{x}-K\right)^{+}, \\
u^{\epsilon}(t, b)=0,
\end{gathered}
$$

where we assume that the risk-free interest rate is zero without loss of generality: for the case of a nonzero constant interest rate $r$, the same derivation is applied if we replace $u^{\epsilon}(t, x)$ and $f\left(e^{x}\right)$ by $\bar{u}^{\epsilon}(t, x):=$ $e^{-r t} u^{\epsilon}(t, x)$ and $\bar{f}\left(e^{x}\right):=e^{-r T} f\left(e^{x}\right)$, respectively.

Next, note that $\sigma_{t}^{0}=\sigma_{0}$, and $\left.X^{0} \equiv X^{\epsilon}\right|_{\epsilon=0}$ follows

$$
d X_{t}^{0}=-\frac{\left(\sigma_{0}\right)^{2}}{2} d t+\sigma_{0} d W_{t}^{1} ; X_{0}^{\epsilon}=x
$$

The associated generator with $X^{0}$ is

$$
\mathcal{L}^{0}=\frac{\left(\sigma_{0}\right)^{2}}{2} \partial_{x x}-\frac{\left(\sigma_{0}\right)^{2}}{2} \partial_{x}
$$

The expansion up to the first order of the PDE related to the down-and-out barrier call option price is obtained by a formal expansion;

$$
\left(\partial_{t}+\mathcal{L}^{0}+\epsilon \mathcal{L}^{1}+\cdots\right)\left(u^{0}+\epsilon u^{1}+\cdots\right)=0
$$

with appropriate boundary conditions for each order as follows:

$$
\begin{gathered}
\left(\partial_{t}+\mathcal{L}^{0}\right) u^{0}=0, \\
u^{0}(T, x)=f\left(e^{x}\right), \\
u^{0}(t, b)=0, \\
\left(\partial_{t}+\mathcal{L}^{0}\right) u^{1}(t, x)=-\mathcal{L}^{1} u^{0}(t, x) x>b, t>0 \\
u^{1}(T, x)=0, \\
u^{1}(t, b)=0,
\end{gathered}
$$

where

$$
\mathcal{L}^{1}=\rho\left(\sigma_{0}\right)^{2} \frac{\partial^{2}}{\partial x \partial \sigma_{0}}+\alpha \frac{\partial}{\partial x}+\lambda\left(\theta-\sigma_{0}\right) \frac{\partial}{\partial \sigma_{0}} .
$$

First, (by an approximation procedure $f_{n} \rightarrow(\cdot-K)^{+}$in $\mathcal{S}^{\prime}$ as $n \rightarrow \infty$ ) we know the formula for the down-and-out call price under Black-Scholes model $(\epsilon=0)$ as

$$
u^{0}(t, x)=C^{B S}\left(T-t, e^{x}, \sigma, 0,0, K\right)-\left(\frac{e^{x}}{B}\right) C^{B S}\left(T-t, \frac{B^{2}}{e^{x}}, \sigma, 0,0, K\right),
$$


where $C^{B S}(T-t, z, \sigma, r, \delta, K)$ denotes the Black-Scholes price with time-to-maturity $(T-t)$, spot price $z$, volatility $\sigma$, interest rate $r$, dividend yield $\delta(=r-\alpha)$ and strike price $K$ :

$$
C^{B S}(T-t, z, \sigma, r, \delta, K)=e^{-\delta(T-t)} z N\left(d_{1}(z)\right)-e^{-r(T-t)} K N\left(d_{2}(z)\right),
$$

where

$$
\begin{aligned}
N(y) & =\int_{-\infty}^{y} e^{\left(-y^{2} / 2\right)} d y, \\
d_{1}(z) & =\frac{\log (z / K)+\alpha(T-t)}{\sigma \sqrt{T-t}}+\frac{\sigma^{2}}{2} \sqrt{T-t}, \\
d_{2}(z) & =d_{1}(z)-\sigma \sqrt{T-t} .
\end{aligned}
$$

Hereafter, we will use an abbriviated notation $C^{B S}(T-t, z, \sigma, K)$ for $C^{B S}(T-t, z, \sigma, 0,0, K)$.

Note that

$$
\begin{aligned}
\frac{\partial^{2}}{\partial x \partial \sigma_{0}} u^{0}(t, x) & =\sigma_{0}(T-t)\left(\frac{\partial^{3}}{\partial x^{3}}-\frac{\partial^{2}}{\partial x^{2}}\right) u^{0}(t, x), \\
\frac{\partial}{\partial \sigma_{0}} u^{0}(t, x) & =\sigma_{0}(T-t)\left(\frac{\partial^{2}}{\partial x^{2}}-\frac{\partial}{\partial x}\right) u^{0}(t, x) .
\end{aligned}
$$

Thus,

$$
\begin{aligned}
\mathcal{L}^{1} u^{0}(t, x) & =\rho \sigma_{0}^{3}(T-t)\left(\frac{\partial^{3}}{\partial x^{3}}-\frac{\partial^{2}}{\partial x^{2}}\right) u^{0}(t, x) \\
& +\alpha \frac{\partial}{\partial x} u^{0}(t, x) \\
& +\lambda\left(\theta-\sigma_{0}\right) \sigma_{0}(T-t)\left(\frac{\partial^{2}}{\partial x^{2}}-\frac{\partial}{\partial x}\right) u^{0}(t, x) .
\end{aligned}
$$

Next, let

$$
\begin{aligned}
g(t, x) & :=\frac{(T-t)}{2} \rho\left(\sigma_{0}\right)^{2} \frac{\partial^{2}}{\partial x \partial \sigma_{0}} u^{0}(t, x) \\
& +(T-t) \alpha \frac{\partial}{\partial x} u^{0}(t, x) \\
& +\frac{(T-t)}{2} \lambda\left(\theta-\sigma_{0}\right) \frac{\partial}{\partial \sigma_{0}} u^{0}(t, x) \\
& =\frac{(T-t)^{2}}{2} \rho \sigma_{0}^{3}\left(\frac{\partial^{3}}{\partial x^{3}}-\frac{\partial^{2}}{\partial x^{2}}\right) u^{0}(t, x) \\
& +(T-t) \alpha \frac{\partial}{\partial x} u^{0}(t, x) \\
& +\frac{(T-t)^{2}}{2} \lambda\left(\theta-\sigma_{0}\right) \sigma_{0}\left(\frac{\partial^{2}}{\partial x^{2}}-\frac{\partial}{\partial x}\right) u^{0}(t, x)
\end{aligned}
$$

Then, define

$$
\hat{u}^{1}(t, x):=u^{1}(t, x)-g(t, x)
$$

Hence, we have

$$
\begin{aligned}
& \left(\partial_{t}+\mathcal{L}^{0}\right) \hat{u}^{1}(t, x)=0 ; x>b, t<T \\
& \hat{u}^{1}(T, x)=0 \\
& \hat{u}^{1}(t, b)=-g(t, b) \\
& =-\left.\frac{(T-t)}{2} \rho \sigma_{0}^{2}\left[\left(\frac{\partial^{2}}{\partial x \partial \sigma_{0}}\right) u^{0}(t, x)\right]\right|_{x=b} \\
- & \left.(T-t) \alpha\left[\frac{\partial}{\partial x} u^{0}(t, x)\right]\right|_{x=b} \\
- & \left.\frac{(T-t)}{2} \lambda\left(\theta-\sigma_{0}\right)\left[\frac{\partial}{\partial \sigma_{0}} u^{0}(t, x)\right]\right|_{x=b} .
\end{aligned}
$$


Then, $\hat{u}^{1}(t, x)$ is obtained by

$$
\hat{u}^{1}(t, x)=-\int_{t}^{T} g(s, b) h(s ; x, b) d s,
$$

where $h(s ; x, b)$ is the density function of the first hitting time to $b$ :

$$
h(s ; x, b)=\frac{-(b-x)}{\sqrt{2 \pi \sigma_{0}^{2}(s-t)^{3}}} \exp \left(-\frac{\left\{b-x+\sigma_{0}^{2} / 2(s-t)\right\}^{2}}{2 \sigma_{0}^{2}(s-t)}\right) .
$$

Therefore,

$$
u^{1}(t, x)=\hat{u}^{1}(t, x)+g(t, x) .
$$

Hereafter, we will evaluate $g(t, x)$, more explicitly.

Note first the following relations:

$$
\begin{aligned}
& \frac{\partial}{\partial x}\left[C^{B S}\left(T-t, e^{x}, \sigma_{0}, K\right)\right]=e^{x} N\left(d_{1}(x)\right) \\
& \frac{\partial}{\partial x}\left[\left(\frac{e^{x}}{B}\right) C^{B S}\left(T-t, \frac{B^{2}}{e^{x}}, \sigma_{0}, K\right)\right]=\left(\frac{e^{x}}{B}\right) C^{B S}\left(T-t, \frac{B^{2}}{e^{x}}, \sigma_{0}, K\right)-B N\left(c_{1}(x)\right), \\
& \frac{\partial}{\partial \sigma_{0}}\left[C^{B S}\left(T-t, e^{x}, \sigma_{0}, K\right)\right]=e^{x} n\left(d_{1}(x)\right) \sqrt{T-t}, \\
& \frac{\partial}{\partial \sigma_{0}}\left[\left(\frac{e^{x}}{B}\right) C^{B S}\left(T-t, \frac{B^{2}}{e^{x}}, \sigma_{0}, K\right)\right]=B n\left(c_{1}(x)\right) \sqrt{T-t}, \\
&=\rho\left(\sigma_{0}\right)^{2} e^{x} n\left(d_{1}(x)\right)\left(-d_{2}(x)\right) \frac{1}{\sigma_{0}} \\
&=\rho \sigma_{0} e^{x} n\left(d_{1}(x)\right)\left(-d_{2}(x)\right), \\
& \rho\left(\sigma_{0}\right)^{2} \frac{\partial^{2}}{\partial x \partial \sigma_{0}} C^{B S}\left(T-t, e^{x}, \sigma_{0}, K\right)=\rho\left(\sigma_{0}\right)^{2} e^{x} n\left(d_{1}(x)\right) \sqrt{T-t}\left\{1+\left(-d_{1}(x)\right) \frac{1}{\sigma_{0} \sqrt{T-t}}\right\} \\
&=\rho\left(\sigma_{0}\right)^{2} B n\left(c_{1}(x)\right)\left(-c_{1}(x)\right) \frac{-1}{\partial_{0} \sqrt{T-t}} \sqrt{T-t} \\
&=\rho\left(\sigma_{0}\right)^{2} B n\left(c_{1}(x)\right)\left(c_{1}(x)\right) \frac{1}{\sigma_{0}} \\
&=\rho \sigma_{0} B n\left(c_{1}(x)\right)\left(c_{1}(x)\right),
\end{aligned}
$$

where

$$
\begin{aligned}
n(x) & =\frac{1}{\sqrt{2 \pi}} \exp \left(\frac{-x^{2}}{2}\right), \\
d_{1}(x) & =\frac{\log \left(e^{x} / K\right)+\sigma_{0}^{2}(T-t) / 2}{\sigma_{0} \sqrt{T-t}}, \\
d_{2}(x) & =d_{1}(x)-\sigma_{0} \sqrt{T-t} \\
c_{1}(x) & =\frac{\ln \left(B^{2} / e^{x} K\right)+\sigma_{0}^{2}(T-t) / 2}{\sigma_{0} \sqrt{(T-t)}} .
\end{aligned}
$$

Then,

$$
\begin{aligned}
g(t, x) & =(T-t) \alpha\left[e^{x} N\left(d_{1}(x)\right)-\left(\frac{e^{x}}{B}\right) C^{B S}\left(T-t, \frac{B^{2}}{e^{x}}, \sigma_{0}, K\right)+B N\left(c_{1}(x)\right)\right] \\
& +\frac{(T-t)}{2} \lambda\left(\theta-\sigma_{0}\right)\left(e^{x} n\left(d_{1}(x)\right) \sqrt{T-t}-B n\left(c_{1}(x)\right) \sqrt{T-t}\right) \\
& -\frac{(T-t)}{2} \rho \sigma_{0}\left\{e^{x} n\left(d_{1}(x)\right) d_{2}(x)+B n\left(c_{1}(x)\right) c_{1}(x)\right\}
\end{aligned}
$$




$$
\begin{aligned}
g(t, b) & =(T-t) \alpha\left[B N\left(d_{1}(b)\right)-\left(\frac{B}{B}\right) C^{B S}\left(T-t, \frac{B^{2}}{B}, \sigma_{0}, K\right)+B N\left(d_{1}(b)\right)\right] \\
& +\frac{(T-t)}{2} \lambda\left(\theta-\sigma_{0}\right)\left(B n\left(d_{1}(b)\right) \sqrt{T-t}-B n\left(d_{1}(b)\right) \sqrt{T-t}\right) \\
& +\frac{(T-t)}{2} \rho \sigma_{0} B n\left(d_{1}(b)\right)\left\{-\left[\left(d_{1}(b)+d_{2}(b)\right]\right\}\right. \\
& =\alpha(T-t)\left[2 B N\left(d_{1}(b)\right)-C^{B S}\left(T-t, B, \sigma_{0}, K\right)\right] \\
& +\frac{(T-t)}{2} \rho \sigma_{0} B \frac{1}{\sqrt{2 \pi}} \exp \left(-\frac{\left\{b-\log K+\frac{1}{2} \sigma_{0}^{2}(T-t)\right\}^{2}}{2 \sigma_{0}^{2}(T-t)}\right)\left(\frac{-2(b-\log K)}{\sigma_{0} \sqrt{T-t}}\right)
\end{aligned}
$$

where

$$
\begin{aligned}
& d_{1}(b)=c_{1}(b)=\frac{\log \left(e^{b} / K\right)}{\sigma_{0} \sqrt{T-t}}+\frac{1}{2} \sigma_{0} \sqrt{T-t} \\
& d_{2}(b)=\frac{\log \left(e^{b} / K\right)}{\sigma_{0} \sqrt{T-t}}-\frac{1}{2} \sigma_{0} \sqrt{T-t}
\end{aligned}
$$

In particular, if the drifts of the underlying asset and its volatility processes are zero, that is $\alpha=0$ and $\lambda=0$, we have

$$
\begin{aligned}
\hat{u}^{1}(t, x)= & \left.-\int_{t}^{T} g(s, b)\right) h(s ; x, b) d s \\
= & -\int_{t}^{T} \frac{(T-s)}{2} \rho \sigma_{0} B \frac{1}{\sqrt{2 \pi}} e^{-\frac{\left\{b-\log K+\frac{1}{2} \sigma_{0}^{2}(T-t)\right\}^{2}}{2 \sigma_{0}^{2}(T-s)}}\left(\frac{-2(b-\log K)}{\left.\sigma_{0} \sqrt{T-s}\right)}\right. \\
& \times \frac{-(b-x)}{\sqrt{2 \pi \sigma_{0}^{2}(s-t)^{3}}} e^{-\frac{\left\{(b-x)+\left(\sigma_{0}^{2} / 2\right)(s-t)\right\}^{2}}{2 \sigma_{0}^{2}(s-t)}} d s \\
= & -\rho \sigma_{0} B \frac{(\log (B / K)(\log (B / S))}{\pi \sigma_{0}^{2}} \\
& \times \frac{1}{2} \int_{t}^{T}(T-s)^{1 / 2} \frac{1}{(s-t)^{3 / 2}} e^{\frac{-\left\{\log B / K+\frac{1}{2} \sigma_{0}^{2}(T-s)\right\}^{2}}{2 \sigma_{0}^{2}(T-s)}} e^{\frac{-\left\{\log B / S+\frac{1}{2} \sigma_{0}^{2}(s-t)\right\}^{2}}{2 \sigma_{0}^{2}(s-t)}} d s
\end{aligned}
$$

and

$$
\begin{aligned}
& u^{1}(t, x) \\
= & \frac{1}{2}(T-t) \rho \sigma_{0}\left\{e^{x} n\left(d_{1}(x)\right)\left(-d_{2}(x)\right)-B n\left(c_{1}(x)\right)\left(c_{1}(x)\right)\right\} \\
& -\rho \sigma_{0} B \frac{(\log (B / K)(\log (B / S))}{\pi \sigma_{0}^{2}} \\
& \times \frac{1}{2} \int_{t}^{T}(T-s)^{1 / 2} \frac{1}{(s-t)^{3 / 2}} e^{\frac{-\left\{\log B / K+\frac{1}{2} \sigma_{0}^{2}(T-s)\right\}^{2}}{2 \sigma_{0}^{2}(T-s)}} e^{\frac{-\left\{\log B / S+\frac{1}{2} \sigma_{0}^{2}(s-t)\right\}^{2}}{2 \sigma_{0}^{2}(s-t)}} d s .
\end{aligned}
$$

Finally, we show a simple numerical example of European down-and-out barrier call prices as an illustrative purpose. Let $f\left(e^{x}\right)=\left(e^{x}-K\right)^{+}$, and the parameters of the model are specified as follows:

$$
\begin{aligned}
& S_{0}=100, r=\alpha=\lambda=0, \sigma_{0}=15 \%, \epsilon=20 \%, \rho=-0.5, \\
& B(\text { barrier })=95, T=0.5, K=100,102,105 .
\end{aligned}
$$

Then, the result is shown in Table 1.

Here, the numbers in the parentheses show the error rates (\%) relative to the benchmark prices, which are computed by Monte Carlo simulations with 100,000 time steps and 1,000,000 trials.

Apparently, our approximation formula improves the accuracies comparing with the Black-Scholes barrier formula. 
Table 1: Down-and-Out Barrier Option

\begin{tabular}{|c|c|c|c|}
\hline Strike & Benchmark & Our Approximation & Barrier Black-Scholes \\
\hline \hline 100 & 3.261 & $3.258(-0.09 \%)$ & $3.290(0.90 \%)$ \\
102 & 2.640 & $2.639(-0.02 \%)$ & $2.686(1.78 \%)$ \\
105 & 1.841 & $1.841(0.01 \%)$ & $1.911(3.77 \%)$ \\
\hline
\end{tabular}

\section{Short-Time Heat Kernel Asymptotic Expansion}

This section derives a short-time asymptotic expansion under multi-dimensional diffusion setting: in particular, the expansion formula developed in Theorem 3.1 is effectively applied.

Consider the following SDE on $\mathbf{R}^{n}$ over the $d$-dimensional Wiener space $(\mathcal{W}, P)$.

$$
\begin{aligned}
d X_{t}^{i} & =\sum_{k=1}^{d} V_{k}^{i}\left(X_{t}\right) \circ d W_{t}^{k}+V_{0}^{i}\left(X_{t}\right) d t, \\
X_{0}^{i} & =x_{0}^{i} \in \mathbf{R}, i=1, \cdots, n .
\end{aligned}
$$

where $V_{k}=\left(V_{k}^{1}, \cdots, V_{k}^{n}\right)$ with $V_{k}^{i} \in C_{b}^{\infty}$. We assume that $\sigma(x)=\left[\sigma^{i j}(x)\right]$ where $\sigma^{i j}(x)=\sum_{k=1}^{d} V_{k}^{i}(x) V_{k}^{j}(x)$ is positive definite at $x=x_{0}$. We also define $\hat{V}_{k}$ as

$$
\hat{V}_{k}=\sum_{i=1}^{n} V_{k}^{i}(x) \frac{\partial}{\partial x_{i}}, \quad k=0,1, \cdots, d .
$$

and

$$
\mathcal{L}=\frac{1}{2} \sum_{k=1}^{d} \hat{V}_{k}^{2}+\hat{V}_{0}
$$

Let $\mathbf{i}=\left(i_{1}, \cdots, i_{m}\right) \in\{0,1, \cdots, d\}^{m}$, we set $\alpha(\mathbf{i})=\#\left\{i: i_{l}=0\right\}$ and $\|\mathbf{i}\|=\alpha(\mathbf{i})+m$. The following stochastic Taylor expansion holds (e.g. p.4 in Baudoin (2009)):

$$
\begin{aligned}
X_{t}= & x_{0}+\sum_{k=1}^{N} \sum_{\mathbf{i},\|\mathbf{i}\|=k}\left(\hat{V}_{i_{k}} \circ \cdots \circ \hat{V}_{i_{2}}\right)\left(V_{i_{1}}\right)\left(x_{0}\right) \int_{0}^{t} \circ d W_{t_{1}}^{i_{1}} \circ \int_{0}^{t_{1}} \circ d W_{t_{2}}^{i_{2}} \cdots \int_{0}^{t_{m-1}} \circ d W_{t_{m}}^{i_{m}} \\
& +\mathbf{R}_{N}(t, x),
\end{aligned}
$$

for some remainder term $\mathbf{R}_{N}(t, x)$ which satisfies

$$
\sup _{x \in \mathbf{R}^{n}} E\left[\mathbf{R}_{N}(t, x)^{2}\right]^{1 / 2} \leq C_{N} t^{(N+1) / 2} \sup _{\mathbf{i}, k+\alpha(\mathbf{i})=N+1 \text { or } N+2}\left\|\left(\hat{V}_{i_{k}} \circ \cdots \circ \hat{V}_{i_{2}}\right)\left(V_{i_{1}}\right)\right\|_{\infty} .
$$

We first consider the scaling SDE in order to obtain a short-time heat kernel expansion:

$$
\begin{aligned}
d X_{t}^{\epsilon} & =\epsilon \sum_{l=1}^{d} V_{k}\left(X_{t}^{\epsilon}\right) \circ d W_{t}^{k}+\epsilon^{2} V_{0}\left(X_{t}^{\epsilon}\right) d t \\
X_{0} & =x_{0} \in \mathbf{R}^{n},
\end{aligned}
$$

where $\epsilon \in(0,1]$. Note that $X_{t}^{\epsilon}$ is equivalent in law to $X_{\epsilon^{2} t}^{1}$, i.e.

$$
X_{t}^{\epsilon} \sim^{\mathcal{L}} X_{\epsilon^{2} t}^{1}
$$

and that $X_{1}^{\epsilon}$ has an asymptotic expansion:

$$
X_{1}^{\epsilon} \sim x_{0}+\sum_{k=1}^{\infty} \epsilon^{k} X_{1}^{(k)} \quad \text { in } \quad \mathbf{D}^{\infty}\left(\mathbf{R}^{n}\right),
$$


where $X_{1}^{(k)}=\left(X_{11}^{(k)}, \cdots, X_{n 1}^{(k)}\right), k \in \mathbf{N}$ is expressed as the coefficient in the stochastic Taylor expansion at $t=1$, i.e.

$$
X_{i 1}^{(k)}=\sum_{\mathbf{i},\|\mathbf{i}\|=k}\left(\hat{V}_{i_{m}} \circ \cdots \circ \hat{V}_{i_{2}}\right)\left(V_{i_{1}}^{i}\right)\left(x_{0}\right) \int_{0}^{1} \circ d W_{t_{1}}^{i_{1}} \circ \int_{0}^{t_{1}} \circ d W_{t_{2}}^{i_{2}} \cdots \int_{0}^{t_{m}-1} \circ d W_{t_{m}}^{i_{m}} .
$$

Next, set

$$
Y_{1}^{\sqrt{t}}=f_{\sqrt{t}}\left(X_{1}^{\sqrt{t}}\right):=\frac{1}{\sqrt{t}}\left(X_{1}^{\sqrt{t}}-x_{0}\right)
$$

Then, we have

$$
p^{X}\left(t, x_{0}, x\right)=p^{X^{\sqrt{t}}}\left(1, x_{0}, x\right)=p^{Y^{\sqrt{t}}}\left(1,0, \frac{x-x_{0}}{t^{1 / 2}}\right) t^{-n / 2} .
$$

Note also that the $(i, j)$-element of the Malliavin covariance matrix of $Y_{1}^{0}=\sum_{k=1}^{d} \int_{0}^{1} V_{k}\left(x_{0}\right) \circ d W_{t}^{k}$ is given as:

$$
\begin{aligned}
\sigma_{Y_{1}^{0}}^{i j} & =\sum_{k=1}^{d} \int_{0}^{1} D_{t, k} Y_{i 1}^{0} D_{t, k} Y_{j 1}^{0} d t \\
& =\sum_{k=1}^{d} V_{k}^{i}\left(x_{0}\right) V_{k}^{j}\left(x_{0}\right)=\sigma^{i j}\left(x_{0}\right) .
\end{aligned}
$$

Since $Y_{1}^{\sqrt{t}}$ is uniformly non-degenerate by the assumption that $\sigma\left(x_{0}\right)$ is positive definite, the smooth density, $p^{Y^{\sqrt{t}}}\left(1, y_{0}, y\right)$ for the law of $Y_{1}^{\sqrt{t}}$ exists.

Thus, $p^{Y^{\sqrt{t}}}\left(1, y_{0}, y\right)$ has an asymptotic expansion by setting $\epsilon=\sqrt{t}$ for $Y^{\epsilon}$, where

$$
Y_{1}^{\epsilon}:=\frac{\left(X_{1}^{\epsilon}-x_{0}\right)}{\epsilon} \sim \sum_{i=1}^{\infty} \epsilon^{i-1} X_{1}^{(i)} \text { in } \mathbf{D}^{\infty}\left(\mathbf{R}^{n}\right) .
$$

In particular,

$$
Y_{1}^{0}=X_{1}^{(1)}=\sum_{k=1}^{d} \int_{0}^{1} V_{k}\left(x_{0}\right) \circ d W_{t}^{k} .
$$

Let $Y_{i 1}^{\epsilon}$ denotes the $i$-th element of $Y_{1}^{\epsilon}$, that is $Y_{1}^{\epsilon}=\left(Y_{11}^{\epsilon}, Y_{21}^{\epsilon}, \cdots, Y_{n 1}^{\epsilon}\right)$, and define $Y_{i 1}^{0, k}, k \in \mathbf{N}, i=1, \cdots, n$ as

$$
Y_{i 1}^{0, k}=\left.\frac{1}{k !} \frac{d^{k}}{d \epsilon^{k}} Y_{i 1}^{\epsilon}\right|_{\epsilon=0}=X_{i 1}^{(k+1)}
$$

Then, applying Theorem 3.1 especially, (3.52), we obtain an asymptotic expansion of $p^{Y^{\epsilon}}(1,0, y)$ :

$$
\begin{aligned}
p^{Y^{\epsilon}}(1,0, y)= & p^{Y^{0}}(1,0, y)\left(\sum_{j=0}^{N} \epsilon^{j} \sum_{k}^{(j)} E\left[H_{k}\left(Y_{1}^{0}, \prod_{l=1}^{k} Y_{\alpha_{l}}^{0, \beta_{l}}\right) \mid Y_{1}^{0}=y\right]\right) \\
& +O\left(\epsilon^{N+1}\right),
\end{aligned}
$$

where

$$
\sum_{k}^{(j)} \equiv \sum_{k=1}^{j} \sum_{\beta_{1}+\cdots+\beta_{k}=j, \beta_{i} \geq 1} \frac{1}{k !} .
$$

Here, it is easily seen that the density of $Y_{1}^{0}$ is given by

$$
p^{Y^{0}}(1,0, y)=(2 \pi)^{-N / 2} \operatorname{det}\left(\sigma\left(x_{0}\right)\right)^{-1 / 2} e^{-\frac{y^{T} \sigma\left(x_{0}\right)-1 y}{2}},
$$

where $\sigma\left(x_{0}\right)=\left(\sum_{k=1}^{d} V_{k}^{i}\left(x_{0}\right) V_{k}^{j}\left(x_{0}\right)\right)_{(1 \leq i, j \leq n)}$.

Consequently, by (5.9), we obtain the following theorem that presents a short-time off-diagonal heat kernel expansion. 
Theorem 5.1 As $t \downarrow 0$, we have a short-time asymptotic expansion of the density $p^{X}\left(t, x_{0}, x\right)$ :

$$
p^{X}\left(t, x_{0}, x\right) \sim \frac{1}{(2 \pi t)^{n / 2}} \operatorname{det} \sigma\left(x_{0}\right)^{-1 / 2} e^{-\frac{\left.\left(x-x_{0}\right)^{T} \sigma\left(x_{0}\right)^{-1}\left(x-x_{0}\right)\right)}{2 t}}\left(\sum_{j=0}^{N} t^{j / 2} \zeta_{j}\left(t^{-1 / 2}\left(x-x_{0}\right)\right)\right),
$$

where $\sigma\left(x_{0}\right)=\left(\sum_{k=1}^{d} V_{k}^{i}\left(x_{0}\right) V_{k}^{j}\left(x_{0}\right)\right)_{(1 \leq i, j \leq n)}$,

and $\zeta_{j}\left(t^{-1 / 2}\left(x-x_{0}\right)\right)$ is the $j$-th push-down of the Malliavin weights ( $j$-th PDE weights in MalliavinThalmaier (2006)) defined by

$$
\begin{aligned}
\zeta_{j}\left(t^{-1 / 2}\left(x-x_{0}\right)\right) & =\sum_{k}^{[j]} E\left[H_{k}\left(X_{1}^{(1)}, \prod_{l=1}^{k} X_{\alpha_{l} 1}^{\left(\beta_{l}\right)}\right) \mid X_{1}^{(1)}=t^{-1 / 2}\left(x-x_{0}\right)\right] \\
& =\sum_{k}^{(j)} E\left[H_{k}\left(Y_{1}^{0}, \prod_{l=1}^{k} Y_{\alpha_{l} 1}^{0, \beta_{l}}\right) \mid Y_{1}^{0}=t^{-1 / 2}\left(x-x_{0}\right)\right] .
\end{aligned}
$$

Here, $Y_{1}^{0}$ and $Y_{i 1}^{0, k}$ are given by (5.12) and (5.13), respectively, and $X_{1}^{(1)}$ and $X_{i 1}^{(k)}$ are given by

$$
\begin{aligned}
X_{1}^{(1)} & =\sum_{k=0}^{d} \int_{0}^{1} V_{k}\left(x_{0}\right) \circ d W_{t}^{k}, \\
X_{i 1}^{(k)} & =\sum_{\mathbf{i},\|\mathbf{i}\|=k}\left(\hat{V}_{i_{m}} \circ \cdots \circ \hat{V}_{i_{2}}\right)\left(V_{i_{1}}^{i}\right)\left(x_{0}\right) \int_{0}^{1} \circ d W_{t_{1}}^{i_{1}} \circ \int_{0}^{t_{1}} \circ d W_{t_{2}}^{i_{2}} \cdots \int_{0}^{t_{m-1}} \circ d W_{t_{m}}^{i_{m}} .
\end{aligned}
$$

Also,

$$
\sum_{k}^{[j]} \equiv \sum_{k=1}^{j} \sum_{\beta_{1}+\cdots+\beta_{k}=j, \beta_{i} \geq 2} \frac{1}{k !}
$$

and

$$
\sum_{k}^{(j)} \equiv \sum_{k=1}^{j} \sum_{\beta_{1}+\cdots+\beta_{k}=j, \beta_{i} \geq 1} \frac{1}{k !}
$$

Remark 5.1 In the diagonal case, the diagonal heat kernel $p^{X}\left(t, x_{0}, x_{0}\right)$ is approximated by

$$
\frac{1}{(2 \pi t)^{n / 2}} \operatorname{det} \sigma\left(x_{0}\right)^{-1 / 2}\left(\sum_{j=0}^{N} t^{j} \zeta_{j}(0)\right)
$$

where

$$
\begin{aligned}
\zeta_{j}(0) & =\sum_{k}^{[j]} E\left[H_{k}\left(X_{1}^{(1)}, \prod_{l=1}^{k} X_{\alpha_{l} 1}^{\left(\beta_{l}\right)}\right) \mid X_{1}^{(1)}=0\right] \\
& =\sum_{k}^{(j)} E\left[H_{k}\left(Y_{1}^{0}, \prod_{l=1}^{k} Y_{\alpha_{l} 1}^{0, \beta_{l}}\right) \mid Y_{1}^{0}=0\right] .
\end{aligned}
$$

Next, we provide alternative methods to obtain the coefficients of the expansion.

Let $\mathcal{A}$ be the perturbed generator associated with (5.6):

$$
\mathcal{A}=\frac{1}{2} \sum_{k=1}^{d}\left(\epsilon \hat{V}_{k}\right)^{2}+\epsilon^{2} \hat{V}_{0}
$$

Then, the generator $\mathcal{L}^{\epsilon}$ associated with the process after the transformation, $Y_{t}^{\epsilon}=\frac{\left(X_{t}^{\epsilon}-x_{0}\right)}{\epsilon}$ is given by

$$
\mathcal{L}^{\epsilon}=\frac{1}{2} \sum_{k=1}^{d}\left(L_{k}^{\epsilon}\right)^{2}+\epsilon L_{0}^{\epsilon} .
$$


where

$$
L_{k}^{\epsilon}=\sum_{i=1}^{n} V_{k}^{i}\left(x_{0}+\epsilon y\right) \frac{\partial}{\partial y_{i}}, \quad k=0,1, \cdots, d .
$$

Hence, by applying (3.48) in Theorem 3.1, we have for $f \in C_{b}\left(\mathbf{R}^{n}\right)$,

$$
\mathbf{P}_{1}^{\epsilon} f(0)=\mathbf{P}_{1}^{0} f(0)+\sum_{j=1}^{N} \epsilon^{j} \xi_{j}(y)+\epsilon^{N+1} R_{N}(y),
$$

where

$$
\begin{aligned}
& \xi_{j}(y) \\
& =\left.\sum_{k=1}^{j} \sum_{\beta_{1}+\cdots+\beta_{k}=j, \beta_{i} \geq 1} \int_{0}^{1} \int_{0}^{t_{1}} \cdots \int_{0}^{t_{k}} \mathbf{P}_{\left(1-t_{1}\right)}^{0} \mathcal{L}^{\beta_{1}} \mathbf{P}_{\left(t_{1}-t_{2}\right)}^{0} \mathcal{L}^{\beta_{2}} \cdots \mathbf{P}_{\left(t_{k}-t_{k-1}\right)}^{0} \mathcal{L}^{\beta_{k}} \mathbf{P}_{t_{k}}^{0} f\left(y_{0}\right) d t_{k} \cdots d t_{2} d t_{1}\right|_{y_{0}=0},
\end{aligned}
$$

with $\mathcal{L}^{k}:=\left.\frac{1}{k !} \frac{d^{k}}{d \epsilon^{k}} \mathcal{L}^{\epsilon}\right|_{\epsilon=0}, k \in \mathbf{N}, i=1, \cdots, n$.

\section{Applications of Short-Time Asymptotic Expansion}

This section shows three examples of Theorem 5.1 in the previous section. In particular, we explicitly derive short-time asymptotic expansions under stochastic volatility model with log-normal local volatility and general local-stochastic volatility models. Moreover, we applies (5.17) and (5.26) in Section 4 to computing the coefficients in the expansions. In addition, for local volatility model in Section 6.1 and Appendix, we compute the expansion coefficients $\xi_{i}(y)(i \in \mathbf{N}), j=1,2$ in (5.26) by using Lie brackets.(Lie bracket $[A, Z]$ stands for $[A, Z]=A Z-Z A$ where $A$ and $Z$ are vector fields.)

\subsection{Short-Time Asymptotic Expansion for Local Volatility Model}

Consider the following time-homogenous local volatility model.

$$
\begin{aligned}
d X_{t} & =\mu\left(X_{t}\right) d t+\sigma\left(X_{t}\right) d W_{t} \\
X_{0} & =x_{0} .
\end{aligned}
$$

Proposition 6.1 We $t \downarrow 0$, we have

$$
p\left(t, x_{0}, x\right) \sim \frac{1}{\sqrt{2 \pi \sigma\left(x_{0}\right)^{2} t}} \exp \left\{-\frac{\left(x-x_{0}\right)^{2}}{\sigma\left(x_{0}\right)^{2} t}\right\}\left(1+\sqrt{t} \vartheta_{1}\left(t, x_{0}, x\right)+t \vartheta_{2}\left(t, x_{0}, x\right)\right)
$$

where

$$
\begin{aligned}
& \vartheta_{1}\left(t, x_{0}, x\right) \\
= & \mu\left(x_{0}\right) \frac{h_{1}\left(\left(x-x_{0}\right) / \sqrt{t}, \sigma^{2}\left(x_{0}\right)\right.}{\sigma^{2}\left(x_{0}\right)}+\frac{1}{2} \sigma\left(x_{0}\right)^{3} \partial \sigma\left(x_{0}\right) \frac{h_{3}\left(\left(x-x_{0}\right) / \sqrt{t}, \sigma^{2}\left(x_{0}\right)\right)}{\left(\sigma^{2}\left(x_{0}\right)\right)^{3}},
\end{aligned}
$$

and

$$
\begin{aligned}
& \vartheta_{2}\left(t, x_{0}, x\right) \\
= & \left\{\frac{1}{8} \partial \sigma\left(x_{0}\right)^{2} \sigma^{6}\left(x_{0}\right) \frac{h_{6}\left(\left(x-x_{0}\right) / \sqrt{t}, \sigma^{2}\left(x_{0}\right)\right)}{\left(\sigma^{2}\left(x_{0}\right)\right)^{6}}\right. \\
& +\frac{1}{6}\left(\partial^{2} \sigma\left(x_{0}\right) \sigma\left(x_{0}\right)^{5}+4 \partial \sigma\left(x_{0}\right)^{2} \sigma\left(x_{0}\right)+3 \mu\left(x_{0}\right) \partial \sigma\left(x_{0}\right)\right) \frac{h_{4}\left(\left(x-x_{0}\right) / \sqrt{t},\left(\sigma^{2}\left(x_{0}\right)\right)\right)}{\left(\sigma^{2}\left(x_{0}\right)\right)^{4}} \\
& \left.+\frac{1}{4}\left(\partial^{2} \sigma\left(x_{0}\right) \sigma\left(x_{0}\right)^{3}+2 \mu^{\prime}\left(x_{0}\right) \sigma\left(x_{0}\right)^{2}+2 \mu\left(x_{0}\right) \partial \sigma\left(x_{0}\right) \sigma\left(x_{0}\right)+\partial \sigma\left(x_{0}\right)^{2} \sigma\left(x_{0}\right)^{2}+2 \mu\left(x_{0}\right)^{2}\right) \frac{h_{2}\left(\left(x-x_{0}\right) / \sqrt{t}, \sigma^{2}\left(x_{0}\right)\right)}{\left(\sigma^{2}\left(x_{0}\right)\right)^{2}}\right\} .
\end{aligned}
$$

Here, $h_{n}(x, \Sigma)$ stands for the Hermite polynomial of degree $n$ with $\Sigma$, that is

$$
h_{n}(x ; \Sigma)=(-\Sigma)^{n} e^{x^{2} /(2 \Sigma)} \frac{d^{n}}{d x^{n}} e^{-x^{2} /(2 \Sigma)} .
$$


Proof 6.1 We apply (5.17) and (5.26) in computation of the coefficients of the expansion.

First, we have the following stochastic Taylor expansion

$$
X_{t}=x_{0}+X_{1 t}+X_{2 t}+X_{3 t}+\mathbf{R}_{3}(t),
$$

where

$$
\begin{aligned}
X_{1 t}= & \int_{0}^{t} \sigma\left(x_{0}\right) d W_{s}, \\
X_{2 t}= & \int_{0}^{t} \mu\left(x_{0}\right) d s+\int_{0}^{t} \partial \sigma\left(x_{0}\right) \int_{0}^{s} \sigma\left(x_{0}\right) d W_{u} d W_{s} . \\
X_{3 t}= & \int_{0}^{t} \partial \mu\left(x_{0}\right) \int_{0}^{s} \sigma\left(x_{0}\right) d W_{u} d s \\
& +\frac{1}{2} \int_{0}^{t} \partial^{2} \sigma\left(x_{0}\right)\left(\int_{0}^{s} \sigma\left(x_{0}\right) d W_{u}\right)^{2} d W_{s} \\
& +\int_{0}^{t} \partial \sigma\left(x_{0}\right) \int_{0}^{s} \mu\left(x_{0}\right) d u d W_{s}+\int_{0}^{t} \partial \sigma\left(x_{0}\right) \int_{0}^{s} \partial \sigma\left(x_{0}\right) \int_{0}^{u} \sigma\left(x_{0}\right) d W_{v} d W_{u} d W_{s},
\end{aligned}
$$

and $\mathbf{R}_{3}(t)$ is a remainder term.

Let $X_{t}^{\epsilon}$ be the solution of the following scaling SDE.

$$
\begin{aligned}
d X_{t}^{\epsilon} & =\epsilon^{2} \mu\left(X_{t}^{\epsilon}\right) d t+\epsilon \sigma\left(X_{t}^{\epsilon}\right) d W_{t}, \\
X_{0}^{\epsilon} & =x_{0} .
\end{aligned}
$$

Let $\mathcal{A}^{\epsilon}$ be the generator of $X_{t}^{\epsilon}$ defined by

$$
\mathcal{A}^{\epsilon}=\epsilon^{2} \frac{1}{2} \sigma^{2}(x) \frac{\partial^{2}}{\partial x^{2}}+\epsilon^{2} \mu(x) \frac{\partial}{\partial x} .
$$

Consider a transform

$$
Y_{t}^{\epsilon}=f_{\epsilon}\left(X_{t}^{\epsilon}\right)=\frac{1}{\epsilon}\left(X_{t}^{\epsilon}-x_{0}\right),
$$

then the generator $\mathcal{L}^{\epsilon}$ of $Y_{t}^{\epsilon}$ has the following form

$$
\mathcal{L}^{\epsilon}=\frac{1}{2} \sigma^{2}\left(x_{0}+\epsilon y\right) \frac{\partial^{2}}{\partial y^{2}}+\epsilon \mu\left(x_{0}+\epsilon y\right) \frac{\partial}{\partial y} .
$$

First, we apply the push-down of the Malliavin weights to computing the coefficients of the expansion.

Note that $X_{t}^{\epsilon}$ and $Y_{t}^{\epsilon}$ are expanded in $\mathbf{D}^{\infty}$ as follows.

$$
\begin{aligned}
X_{t}^{\epsilon} & =x_{0}+\epsilon X_{1 t}+\epsilon^{2} X_{2 t}+\epsilon^{3} X_{3 t}+O\left(\epsilon^{4}\right), \\
Y_{t}^{\epsilon} & =Y_{0 t}+\epsilon Y_{1 t}+\epsilon^{2} Y_{2 t}+O\left(\epsilon^{3}\right),
\end{aligned}
$$

where

$$
\begin{aligned}
Y_{0 t}= & X_{1 t}=\left.\frac{\partial}{\partial \epsilon} X_{t}^{\epsilon}\right|_{\epsilon=0}=\int_{0}^{t} \sigma\left(x_{0}\right) d W_{s}, \\
Y_{1 t}= & X_{2 t}=\left.\frac{1}{2 !} \frac{\partial^{2}}{\partial \epsilon^{2}} X_{t}^{\epsilon}\right|_{\epsilon=0} \\
= & \int_{0}^{t} \mu\left(x_{0}\right) d s+\int_{0}^{t} \partial \sigma\left(x_{0}\right) \int_{0}^{s} \sigma\left(x_{0}\right) d W_{u} d W_{s} . \\
Y_{2 t}= & X_{3 t}=\left.\frac{1}{3 !} \frac{\partial^{3}}{\partial \epsilon^{3}} X_{t}^{\epsilon}\right|_{\epsilon=0} \\
= & \int_{0}^{t} \partial \mu\left(x_{0}\right) \int_{0}^{s} \sigma\left(x_{0}\right) d W_{u} d s \\
& +\frac{1}{2} \int_{0}^{t} \partial \partial^{2} \sigma\left(x_{0}\right)\left(\int_{0}^{s} \sigma\left(x_{0}\right) d W_{u}\right)^{2} d W_{s} \\
& +\int_{0}^{t} \partial \sigma\left(x_{0}\right) \int_{0}^{s} \mu\left(x_{0}\right) d u d W_{s}+\int_{0}^{t} \partial \sigma\left(x_{0}\right) \int_{0}^{s} \partial \sigma\left(x_{0}\right) \int_{0}^{u} \sigma\left(x_{0}\right) d W_{v} d W_{u} d W_{s} .
\end{aligned}
$$


Note that $Y_{t}^{\epsilon}$ is uniformly non-degenrate.

The following relation holds,

$$
p^{X}\left(t, x_{0}, x\right)=p^{Y}\left(1, f_{\sqrt{t}}\left(x_{0}\right), f_{\sqrt{t}}(x)\right) \frac{1}{\sqrt{t}} .
$$

1. Using Bismut identity,

$$
\begin{aligned}
& \frac{\partial}{\partial \epsilon} p^{Y^{\epsilon}}(1,0, y) \\
= & \left.\frac{\partial}{\partial \epsilon} E\left[\delta_{y}\left(Y_{1}^{(\epsilon)}\right)\right]\right|_{\epsilon=0}\left(\delta_{y}(\cdot) \text { is a delta function at } y .\right) \\
= & E\left[\delta_{y}\left(Y_{1}^{(0)}\right) H_{1}\left(Y_{1}^{(0)},\left.\frac{\partial}{\partial \epsilon} Y_{1}^{(\epsilon)}\right|_{\epsilon=0}\right)\right] \\
= & E\left[\delta_{y}\left(Y_{1}^{(0)}\right) \frac{1}{\sigma^{2}\left(x_{0}\right)}\left\{\left.\frac{\partial}{\partial \epsilon} Y_{1}^{(\epsilon)}\right|_{\epsilon=0} \int_{0}^{1} \sigma\left(x_{0}\right) d W_{u}-\left.\int_{0}^{1} D_{1, u} \frac{\partial}{\partial \epsilon} Y_{1}^{(\epsilon)}\right|_{\epsilon=0} \sigma\left(x_{0}\right) d u\right\}\right] \\
= & E\left[\delta_{y}\left(Y_{1}^{(0)}\right) \frac{1}{\sigma^{2}\left(x_{0}\right)}\left\{\left(\mu\left(x_{0}\right)+\sigma\left(x_{0}\right) \partial \sigma\left(x_{0}\right) \int_{0}^{1} W_{s} d W_{s}\right) \sigma\left(x_{0}\right) W_{1}-\sigma^{2}\left(x_{0}\right) \partial \sigma\left(x_{0}\right) W_{1}\right\}\right] \\
= & \int_{\mathbf{R}} \delta_{y}(v) E\left[\frac{1}{\sigma^{2}\left(x_{0}\right)}\left\{\left(\mu\left(x_{0}\right)+\sigma\left(x_{0}\right) \partial \sigma\left(x_{0}\right) \int_{0}^{1} W_{s} d W_{s}\right) \sigma\left(x_{0}\right) W_{1}-\sigma^{2}\left(x_{0}\right) \partial \sigma\left(x_{0}\right) W_{1}\right\} \mid \sigma\left(x_{0}\right) W_{1}=v\right] \\
& \times \frac{1}{\sqrt{2 \pi}} e^{-\frac{1}{2} v^{2}} d v \\
= & E\left[\frac{1}{\sigma^{2}\left(x_{0}\right)}\left\{\left(\mu\left(x_{0}\right)+\sigma\left(x_{0}\right) \partial \sigma\left(x_{0}\right) \int_{0}^{1} W_{s} d W_{s}\right) \sigma\left(x_{0}\right) W_{1}-\sigma^{2}\left(x_{0}\right) \partial \sigma\left(x_{0}\right) W_{1}\right\} \mid \sigma\left(x_{0}\right) W_{1}=y\right] \\
& \times \frac{1}{\sqrt{2 \pi \sigma\left(x_{0}\right)^{2}}} e^{-\frac{1}{2}\left(\frac{y}{\sigma\left(x_{0}\right)}\right)^{2}}
\end{aligned}
$$

Note that

$$
D_{1, u} \int_{0}^{1} \sigma\left(x_{0}\right) \partial_{x_{0}} \sigma\left(x_{0}\right) W_{s} d W_{s}=\sigma\left(x_{0}\right) \partial_{x_{0}} \sigma(z)\left\{W_{u}+\int_{u}^{1} d W_{s}\right\}=\sigma\left(x_{0}\right) \partial \sigma\left(x_{0}\right) W_{1}
$$

Note that

$$
E\left[\int_{0}^{1} W_{s} d W_{s} \mid \sigma(z) W_{1}=y\right]=\left(\int_{0}^{1} s d s\right)\left(\frac{y^{2}}{\sigma\left(x_{0}\right)^{4}}-\frac{1}{\sigma\left(x_{0}\right)^{2}}\right)=\frac{1}{2}\left(\frac{y^{2}}{\sigma\left(x_{0}\right)^{4}}-\frac{1}{\sigma\left(x_{0}\right)^{2}}\right) .
$$

Then, we obtain

$$
\begin{aligned}
& \left.\frac{\partial}{\partial \epsilon} E\left[\delta_{y}\left(Y_{1}^{(\epsilon)}\right)\right]\right|_{\epsilon=0} \\
= & \left\{\mu\left(x_{0}\right) \frac{y}{\sigma\left(x_{0}\right)^{2}}+\frac{1}{2} \sigma\left(x_{0}\right)^{3} \partial \sigma\left(x_{0}\right)\left(\frac{y^{3}}{\sigma\left(x_{0}\right)^{6}}-\frac{3 y}{\sigma\left(x_{0}\right)^{4}}\right)\right\} \frac{1}{\sqrt{2 \pi \sigma\left(x_{0}\right)^{2}}} e^{-\frac{1}{2}\left(\frac{y}{\sigma\left(x_{0}\right)}\right)^{2} .} .
\end{aligned}
$$

2. Alternatively, we can evaluate the coefficients of the expansion in the following way.

Let

$$
\begin{aligned}
\mathcal{L}^{0} & =\frac{1}{2} \sigma^{2}\left(x_{0}\right) \frac{\partial^{2}}{\partial y^{2}} \\
\mathcal{L}^{1} & =\sigma\left(x_{0}\right) \partial \sigma\left(x_{0}\right) y \frac{\partial^{2}}{\partial y^{2}}+\mu\left(x_{0}\right) \frac{\partial}{\partial y}, \\
\mathcal{L}^{2} & =\frac{1}{2}\left(\left(\partial \sigma\left(x_{0}\right)\right)^{2}+\sigma\left(x_{0}\right) \partial^{2} \sigma\left(x_{0}\right)\right) y^{2} \frac{\partial^{2}}{\partial y^{2}}+\partial \mu\left(x_{0}\right) y \frac{\partial}{\partial y},
\end{aligned}
$$

then

$$
\left.\left.\frac{\partial}{\partial \epsilon}\right|_{\epsilon=0} \mathbf{P}_{1}^{\epsilon} f\left(y_{0}\right)\right|_{y_{0}=0}=\left.\int_{0}^{1} \mathbf{P}_{(1-s)}^{0} \mathcal{L}^{1} \mathbf{P}_{s}^{0} f\left(y_{0}\right) d s\right|_{y_{0}=0}
$$


Let $h$ be a map $y \mapsto h(y)$ such that

$$
\begin{aligned}
h(y) & =\mathcal{L}^{1} \mathbf{P}_{s} f(y)=\mathcal{L}^{1} \mathbf{P}_{1-(1-s)} f(y) \\
& =\mathcal{L}^{1} E\left[f\left(Y_{1}^{0}\right) \mid Y_{1-s}^{0}=y\right]=\mathcal{L}^{1} \int_{\mathbf{R}} p^{Y^{0}}(s, y, z) f(z) d z \\
& =\left(\sigma\left(x_{0}\right) \partial \sigma\left(x_{0}\right) y \frac{\partial^{2}}{\partial y^{2}}+\mu\left(x_{0}\right) \frac{\partial}{\partial y}\right) \int_{\mathbf{R}} p^{Y^{0}}(s, y, z) f(z) d z .
\end{aligned}
$$

Then, we explicitly evaluate (5.26) for $j=1$.

$$
\begin{aligned}
& \left.\mathbf{P}_{(1-s)}^{0} \mathcal{L}^{1} \mathbf{P}_{s}^{0} f\left(y_{0}\right)\right|_{y_{0}=0} \\
= & \left.\int_{\mathbf{R}} p^{Y^{0}}\left(1-s, y_{0}, y\right) h(y) d y\right|_{y_{0}=0} \\
= & \left.\int_{\mathbf{R}} p^{Y^{0}}\left(1-s, y_{0}, y\right)\left(\mathcal{L}^{1} \int_{\mathbf{R}} p^{Y^{0}}(s, y, z) f(z) d z\right) d y\right|_{y_{0}=0} \\
= & \left.\int_{\mathbf{R}} p^{Y^{0}}\left(1-s, y_{0}, y\right)\left(\left(\sigma\left(x_{0}\right) \partial \sigma\left(x_{0}\right)\left(y-y_{0}\right) \frac{\partial^{2}}{\partial y^{2}}+\mu\left(x_{0}\right) \frac{\partial}{\partial y}\right) \int_{\mathbf{R}} p^{Y^{0}}(s, y, z) f(z) d z\right) d y\right|_{y_{0}=0 .}
\end{aligned}
$$

Note that

$$
p^{Y^{0}}\left(1-s, y_{0}, y\right)\left(y-y_{0}\right)=(1-s) \sigma\left(x_{0}\right)^{2} p^{Y^{0}}\left(1-s, y_{0}, y\right) \frac{\partial}{\partial y_{0}} .
$$

Therefore, we have

$$
\begin{aligned}
& \left.\mathbf{P}_{(1-s)}^{0} \mathcal{L}^{1} \mathbf{P}_{s}^{0} f\left(y_{0}\right)\right|_{y_{0}=0} \\
= & \left.\int_{\mathbf{R}} \frac{\partial}{\partial y_{0}} p^{Y^{0}}\left(1-s, y_{0}, y\right)\left(\left((1-s) \sigma\left(x_{0}\right)^{3} \partial \sigma\left(x_{0}\right) \frac{\partial^{2}}{\partial y^{2}}+\mu\left(x_{0}\right) \frac{\partial}{\partial y}\right) \int_{\mathbf{R}} p^{Y^{0}}(s, y, z) f(z) d z\right) d y\right|_{y_{0}=0} \\
= & \left.\int_{\mathbf{R}} \frac{\partial^{3}}{\partial y^{2} \partial y_{0}} p^{Y^{0}}\left(1-s, y_{0}, y\right)\left(\left((1-s) \sigma\left(x_{0}\right)^{3} \partial \sigma\left(x_{0}\right)\right) \int_{\mathbf{R}} p^{Y^{0}}(s, y, z) f(z) d z\right) d y\right|_{y_{0}=0} \\
& -\left.\int_{\mathbf{R}} \frac{\partial}{\partial y} p^{Y^{0}}\left(1-s, y_{0}, y\right)\left(\mu\left(x_{0}\right) \int_{\mathbf{R}} p^{Y^{0}}(s, y, z) f(z) d z\right) d y\right|_{y_{0}=0} \\
= & \left.(1-s) \sigma\left(x_{0}\right)^{3} \partial \sigma\left(x_{0}\right) \int_{\mathbf{R}} \frac{\partial^{3}}{\partial y_{0}^{3}} p^{Y^{0}}\left(1-s, y_{0}, y\right)\left(\int_{\mathbf{R}} p^{Y^{0}}(s, y, z) f(z) d z\right) d y\right|_{y_{0}=0} \\
& +\left.\mu\left(x_{0}\right) \int_{\mathbf{R}} \frac{\partial}{\partial y_{0}} p^{Y^{0}}\left(1-s, y_{0}, y\right)\left(\int_{\mathbf{R}} p^{Y^{0}}(s, y, z) f(z) d z\right) d y\right|_{y_{0}=0} \\
= & \left.(1-s) \sigma\left(x_{0}\right)^{3} \partial \sigma\left(x_{0}\right) \int_{\mathbf{R}} \frac{\partial^{3}}{\partial y_{0}^{3}}\left(\int_{\mathbf{R}} p^{Y^{0}}\left(1-s, y_{0}, y\right) p^{Y^{0}}(s, y, z) d y\right) f(z) d z\right|_{y_{0}=0} \\
& +\left.\mu\left(x_{0}\right) \int_{\mathbf{R}} \frac{\partial}{\partial y_{0}}\left(\int_{\mathbf{R}} p^{Y^{0}}\left(1-s, y_{0}, y\right) p^{Y^{0}}(s, y, z) d y\right) f(z) d z\right|_{y_{0}=0} \\
= & \left.(1-s) \sigma\left(x_{0}\right)^{3} \partial \sigma\left(x_{0}\right) \frac{\partial^{3}}{\partial y_{0}^{3}} \int_{\mathbf{R}} p^{Y^{0}}\left(1, y_{0}, z\right) f(z) d z\right|_{y_{0}=0}+\left.\mu\left(x_{0}\right) \frac{\partial}{\partial y_{0}} \int_{\mathbf{R}} p^{Y^{0}}\left(1, y_{0}, z\right) f(z) d z\right|_{y_{0}=0} \\
= & \left.(1-s) \sigma\left(x_{0}\right)^{3} \partial \sigma\left(x_{0}\right) \frac{\partial^{3}}{\partial y_{0}^{3}} \mathbf{P}_{1}^{0} f\left(y_{0}\right)\right|_{y_{0}=0}+\left.\mu\left(x_{0}\right) \frac{\partial}{\partial y_{0}} \mathbf{P}_{1}^{0} f\left(y_{0}\right)\right|_{y_{0}=0 .}
\end{aligned}
$$

Then, the first order approximation term is given by

$$
\begin{aligned}
& \left.\int_{0}^{1} \mathbf{P}_{(1-s)}^{0} \mathcal{L}^{1} \mathbf{P}_{s}^{0} f\left(y_{0}\right) d s\right|_{y_{0}=0} \\
= & \left.\left(\int_{0}^{1}(1-s) d s\right) \sigma\left(x_{0}\right)^{3} \partial \sigma\left(x_{0}\right) \frac{\partial^{3}}{\partial y_{0}^{3}} \mathbf{P}_{1}^{0} f\left(y_{0}\right)\right|_{y_{0}=0}+\left.\mu\left(x_{0}\right) \frac{\partial}{\partial y_{0}} \mathbf{P}_{1}^{0} f\left(y_{0}\right)\right|_{y_{0}=0} \\
= & \int_{\mathbf{R}}\left\{\frac{1}{2} \sigma\left(x_{0}\right)^{3} \partial \sigma\left(x_{0}\right)\left(\frac{z^{3}}{\sigma\left(x_{0}\right)^{6}}-\frac{3 z}{\sigma\left(x_{0}\right)^{4}}\right)+\mu\left(x_{0}\right) \frac{z}{\sigma\left(x_{0}\right)^{2}}\right\} p^{Y^{0}}(1,0, z) f(z) d z .
\end{aligned}
$$


3. Moreover, the coefficient is computed by using the Lie bracket.

$$
\begin{aligned}
\int_{0}^{1} \mathbf{P}_{\left(1-t_{1}\right)}^{0} \mathcal{L}^{1} \mathbf{P}_{t_{1}}^{0} f(0) d t_{1} & =\left.\int_{0}^{1}\left(\sum_{i=0}^{\infty} \frac{\left(1-t_{1}\right)^{i}}{i !}\left[\mathcal{L}^{0},\left[\mathcal{L}^{0}, \cdots,\left[\mathcal{L}^{0}, \mathcal{L}^{1}\right]\right]\right]\right) \mathbf{P}_{1}^{0} f\left(y_{0}\right) d t_{1}\right|_{y_{0}=0} \\
& =\left.\left(\mathcal{L}^{1}+\frac{1}{2}\left[\mathcal{L}^{0}, \mathcal{L}^{1}\right]\right) \mathbf{P}_{1}^{0} f\left(y_{0}\right)\right|_{y_{0}=0}
\end{aligned}
$$

because $\left[\mathcal{L}^{0},\left[\mathcal{L}^{0}, \mathcal{L}^{1}\right]\right]=0$ and hence all the terms in (6.12) for $i \geq 2$ are equal to 0.

The Lie bracket $\left[\mathcal{L}^{0}, \mathcal{L}^{1}\right]$ is explicitly computed as follows.

$$
\begin{aligned}
\mathcal{L}^{0} \mathcal{L}^{1} & =\frac{1}{2} \sigma\left(x_{0}\right)^{2} \partial^{2}\left(\sigma\left(x_{0}\right) \partial \sigma\left(x_{0}\right) y \partial^{2}\right) \\
& =\frac{1}{2} \sigma^{3}\left(x_{0}\right) \partial \sigma\left(x_{0}\right)\left(\partial\left(\partial^{2}+y \partial^{3}\right)\right) \\
& =\frac{1}{2} \sigma^{3}\left(x_{0}\right) \partial \sigma\left(x_{0}\right)\left(2 \partial^{3}+y \partial_{x}^{4}\right) . \\
\mathcal{L}^{1} \mathcal{L}^{0} & =\sigma\left(x_{0}\right) \partial \sigma\left(x_{0}\right) y \partial^{2}\left(\frac{1}{2} \sigma\left(x_{0}\right)^{2} \partial^{2}\right) \\
& =\frac{1}{2} \sigma^{3}\left(x_{0}\right) \partial \sigma\left(x_{0}\right) y \partial^{4} .
\end{aligned}
$$

Then

$$
\begin{aligned}
{\left[\mathcal{L}^{0}, \mathcal{L}^{1}\right] } & =\mathcal{L}^{0} \mathcal{L}^{1}-\mathcal{L}^{1} \mathcal{L}^{0} \\
& =\sigma\left(x_{0}\right)^{3} \partial \sigma\left(x_{0}\right) \partial^{3}
\end{aligned}
$$

Then we have

$$
\begin{aligned}
& \left.\left(\mathcal{L}^{1}+\frac{1}{2}\left[\mathcal{L}^{0}, \mathcal{L}^{1}\right]\right) \frac{1}{\sqrt{2 \pi \sigma\left(x_{0}\right)^{2}}} e^{-\frac{1}{2}\left(\frac{y-y_{0}}{\sigma\left(x_{0}\right)}\right)^{2}}\right|_{y_{0}=0} \\
= & \left\{\mu\left(x_{0}\right) \frac{y}{\sigma\left(x_{0}\right)^{2}}+\frac{1}{2} \sigma\left(x_{0}\right)^{3} \partial_{x_{0}} \sigma\left(x_{0}\right)\left(\frac{y^{3}}{\sigma\left(x_{0}\right)^{6}}-\frac{3 y}{\sigma\left(x_{0}\right)^{4}}\right)\right\} \frac{1}{\sqrt{2 \pi \sigma\left(x_{0}\right)^{2}}} e^{-\frac{1}{2}\left(\frac{y}{\sigma\left(x_{0}\right)}\right)^{2}} .
\end{aligned}
$$

The calculation of the second term approximation is given in Appendix.

\subsection{Short Time Asymptotics for Stochastic Volatility Model with Log- normal Local Volatility}

Consider the following stochastic volatility model with log-normal local volatility which includes the Heston type model:

$$
\begin{aligned}
d S_{t} & =r S_{t} d t+\sqrt{v_{t}} S_{t} d W_{t} \\
S_{0} & =s_{0}>0 \\
d v_{t} & =a\left(v_{t}\right) d t+b\left(v_{t}\right) d Z_{t} \\
v_{0} & =v>0
\end{aligned}
$$

where $W_{t}$ and $Z_{t}$ are two standard Brownian motions with correlation $\rho$. We have a short-time expansion of density for the logarithmic process .

Proposition 6.2 When $t \downarrow 0$, we have

$$
p^{X}\left(t, x_{0}, x\right) \sim \frac{1}{\sqrt{2 \pi v_{0} t}} \exp \left(-\frac{\left(x-x_{0}\right)^{2}}{2 v_{0} t}\right)\left\{1+\sqrt{t} w_{1}\left(t, x_{0}, x\right)\right\},
$$

where $x=\log s, x_{0}=\log s_{0}$, and

$$
w_{1}\left(t, x_{0}, x\right)=\frac{1}{4} \rho \sqrt{v_{0}} b\left(v_{0}\right) \frac{h_{3}\left(\left(x-x_{0}\right) / \sqrt{t}, v_{0}\right)}{v_{0}^{3}}+\left(r-\frac{1}{2} v_{0}\right) \frac{h_{1}\left(\left(x-x_{0}\right) / \sqrt{t}, v_{0}\right)}{v_{0}} .
$$


Also, the following approximation formula of the option price holds:

$$
\begin{aligned}
C(t, K) \sim & \int_{\log (K)}^{\infty}\left(e^{x}-K\right) \frac{1}{\sqrt{2 \pi v_{0} t}} \exp \left(-\frac{\left(x-x_{0}\right)^{2}}{2 v_{0} t}\right) d x \\
& +\sqrt{t} \int_{\log (K)}^{\infty}\left(e^{x}-K\right) w_{1}\left(t, x_{0}, x\right) \frac{1}{\sqrt{2 \pi v_{0} t}} \exp \left(-\frac{\left(x-x_{0}\right)^{2}}{2 v_{0} t}\right) d x
\end{aligned}
$$

Proof 6.2 We will apply (5.17) and (5.26) in computation. First, we have the following stochastic Taylor expansion

$$
X_{t}^{\epsilon}=x_{0}+X_{1 t}+X_{2 t}+\mathbf{R}_{2}(t)
$$

where

$$
\begin{aligned}
& X_{1 t}=\int_{0}^{t} \sqrt{v_{0}} d W_{s}, \\
& X_{2 t}=\int_{0}^{t}\left(r-\frac{1}{2} v_{0}\right) d s+\frac{1}{2} \int_{0}^{t} \frac{1}{\sqrt{v_{0}}} \int_{0}^{s} b\left(v_{0}\right) d Z_{u} d W_{s} .
\end{aligned}
$$

Next, we introduce a time scaling parameter $\epsilon=\sqrt{t}$,

$$
\begin{aligned}
d X_{t}^{\epsilon} & =\epsilon^{2}\left(r-\frac{1}{2} v_{t}^{\epsilon}\right) d t+\epsilon \sqrt{v_{t}^{\epsilon}} d W_{t}, \\
d v_{t}^{\epsilon} & =\epsilon^{2} a\left(v_{t}^{\epsilon}\right) d t+\epsilon b\left(v_{t}^{\epsilon}\right) d Z_{t} .
\end{aligned}
$$

The generator of the above diffusion is

$$
\mathcal{A}^{\epsilon}=\epsilon^{2} \frac{1}{2} v \frac{\partial^{2}}{\partial x^{2}}+\epsilon^{2}\left(r-\frac{1}{2} v\right) \frac{\partial}{\partial x}+\epsilon^{2} \rho \sqrt{v} b(v) \frac{\partial^{2}}{\partial x \partial v}+\epsilon^{2} \frac{1}{2} b(v) \frac{\partial^{2}}{\partial v^{2}}+\epsilon^{2} a(v) \frac{\partial}{\partial v} .
$$

Consider a transform $Y=f^{\epsilon}(X)=\frac{1}{\epsilon}\left(X-x_{0}\right)$, then the generator of $(Y, v)$ is given by,

$$
\mathcal{L}^{\epsilon}=\frac{1}{2} v \frac{\partial^{2}}{\partial y^{2}}+\epsilon\left(r-\frac{1}{2} v\right) \frac{\partial}{\partial y}+\epsilon \rho \sqrt{v} b(v) \frac{\partial^{2}}{\partial y \partial v}+\epsilon^{2} \frac{1}{2} b(v) \frac{\partial^{2}}{\partial v^{2}}+\epsilon^{2} a(v) \frac{\partial}{\partial v} .
$$

$X$ and $Y$ are expanded in $\mathbf{D}^{\infty}$,

$$
\begin{aligned}
X_{t}^{\epsilon} & =x_{0}+\epsilon X_{1 t}+\epsilon^{2} X_{2 t}+O\left(\epsilon^{3}\right), \\
Y_{t}^{\epsilon} & =Y_{0 t}+\epsilon Y_{1 t}+O\left(\epsilon^{2}\right),
\end{aligned}
$$

where

$$
\begin{aligned}
Y_{0 t} & =X_{1 t}=\int_{0}^{t} \sqrt{v_{0}} d W_{s} \\
Y_{1 t} & =X_{2 t}=\int_{0}^{t}\left(r-\frac{1}{2} v_{0}\right) d s+\frac{1}{2} \int_{0}^{t} \frac{1}{\sqrt{v_{0}}} \int_{0}^{s} b\left(v_{0}\right) d Z_{u} d W_{s} .
\end{aligned}
$$

Note that

$$
p^{X}\left(t, x_{0}, x\right)=p^{Y^{\sqrt{t}}}\left(1, f^{\sqrt{t}}\left(x_{0}\right), f^{\sqrt{t}}(x)\right) \frac{1}{\sqrt{t}} .
$$

1. Using the Bismut identity, the first order approximation term is given as

$$
\begin{aligned}
& \left.\frac{\partial}{\partial \epsilon} p^{Y^{\epsilon}}\left(1, y_{0}, y\right)\right|_{\epsilon=0} \\
= & E\left[H_{1}\left(Y_{01}, Y_{11}\right) \mid Y_{01}=y\right] p^{Y^{0}}\left(1, y_{0}, y\right) \\
= & \frac{1}{v_{0}} E\left[Y_{11} \int_{0}^{1} \sqrt{v_{0}} d W_{1, t}-\int_{0}^{1} D_{t, 1} Y_{11} \sqrt{v_{0}} d t \mid Y_{01}=y-y_{0}\right] p^{Y^{0}}\left(1, y_{0}, y\right) \\
= & \left\{\frac{1}{4} \rho \sqrt{v_{0}} b\left(v_{0}\right)\left(\frac{\left(y-y_{0}\right)^{3}}{v_{0}^{3}}-3\left(\frac{y-y_{0}}{v_{0}}\right)\right)+\left(r-\frac{1}{2} v_{0}\right)\left(\frac{y-y_{0}}{v_{0}}\right)\right\} p^{Y^{0}}\left(1, y_{0}, y\right) .
\end{aligned}
$$


Then we have a approximation formula of the density

$$
p^{Y^{\sqrt{t}}}\left(1, y_{0}, y\right) \sim \frac{1}{\sqrt{2 \pi v_{0}}} \exp \left\{-\frac{\left(y-y_{0}\right)^{2}}{2 v_{0}}\right\}\left(1+\sqrt{t} \zeta_{1}\left(1, y_{0}, y\right)\right)
$$

where

$$
\zeta_{1}\left(1, y_{0}, y\right)=\frac{1}{4} \rho \sqrt{v_{0}} b\left(v_{0}\right) \frac{h_{3}\left(y, v_{0}\right)}{v_{0}^{3}}+\left(r-\frac{1}{2} v_{0}\right) \frac{h_{1}\left(y, v_{0}\right)}{v_{0}}
$$

By (6.25) and (6.26) with $y_{0}=0$, we have the formula (6.15).

2. Alternatively, we have

$$
\left.\left.\frac{\partial}{\partial \epsilon}\right|_{\epsilon=0} \mathbf{P}_{1} f\left(y_{0}\right)\right|_{y_{0}=0}=\left.\int_{0}^{1} \mathbf{P}_{(1-s)}^{0} \mathcal{L}^{1} \mathbf{P}_{s}^{0} f\left(y_{0}\right) d s\right|_{y_{0}=0}
$$

with

$$
\mathcal{L}^{1}=\left.\frac{\partial}{\partial \epsilon} \mathcal{L}^{\epsilon}\right|_{\epsilon=0}=\left(r-\frac{1}{2} v\right) \frac{\partial}{\partial y}+\rho \sqrt{v} b(v) \frac{\partial^{2}}{\partial y \partial v} .
$$

Note that

$$
\frac{\partial}{\partial v} p^{Y^{0}}(s, y, z)=\frac{1}{2} s \frac{\partial^{2}}{\partial y^{2}} p^{Y^{0}}(s, y, z)
$$

Let $g$ be a map $y \mapsto g(y)$ such that

$$
\begin{aligned}
g(y) & =\mathcal{L}^{1} \mathbf{P}_{s} f(y)=\mathcal{L}^{1} \mathbf{P}_{1-(1-s)} f(y) \\
& =\mathcal{L}^{1} E\left[f\left(Y_{1}^{0}\right) \mid Y_{1-s}^{0}=y\right]=\mathcal{L}^{1} \int_{\mathbf{R}} p^{Y^{0}}(s, y, z) f(z) d z .
\end{aligned}
$$

Then, we explicitly evaluate (5.26) for $j=1$.

$$
\begin{aligned}
& \left.\mathbf{P}_{(1-s)}^{0} \mathcal{L}^{1} \mathbf{P}_{s}^{0} f\left(y_{0}\right)\right|_{y_{0}=0} \\
= & \int_{\mathbf{R}} p^{Y^{0}}\left(1-s, y_{0}, y\right) g(y) d y \\
= & \int_{\mathbf{R}} p^{Y^{0}}\left(1-s, y_{0}, y\right)\left(\mathcal{L}^{1} \int_{\mathbf{R}} p^{Y^{0}}(s, y, z) f(z) d z\right) d y \\
= & \int_{\mathbf{R}} p^{Y^{0}}\left(1-s, y_{0}, y\right)\left(\left(\frac{1}{2} s \rho \sqrt{v} b(v) \frac{\partial^{3}}{\partial y^{3}}+\left(r-\frac{1}{2} v\right) \frac{\partial}{\partial y}\right) \int_{\mathbf{R}} p^{Y^{0}}(s, y, z) f(z) d z\right) d y \\
= & \left.\frac{1}{2} s \rho \sqrt{v} b(v) \frac{\partial^{3}}{\partial y_{0}^{3}} \mathbf{P}_{1} f\left(y_{0}\right)\right|_{y_{0}=0}+\left.\left(r-\frac{1}{2} v\right) \frac{\partial}{\partial y_{0}} \mathbf{P}_{1} f\left(y_{0}\right)\right|_{y_{0}=0}
\end{aligned}
$$

Therefore,

$$
\begin{aligned}
& \left.\int_{0}^{1} \mathbf{P}_{(1-s)}^{0} \mathcal{L}^{1} \mathbf{P}_{s}^{0} f\left(y_{0}\right) d s\right|_{y_{0}=0} \\
= & \int_{\mathbf{R}}\left\{\frac{1}{4} \rho \sqrt{v_{0}} b\left(v_{0}\right)\left(\frac{y^{3}}{v_{0}^{3}}-3\left(\frac{y}{v_{0}}\right)\right)+\left(r-\frac{1}{2} v_{0}\right)\left(\frac{y}{v_{0}}\right)\right\} p^{Y^{0}}(1,0, y) d y .
\end{aligned}
$$

The second order approximation can be obtained in the similar manner.

\subsection{Short Time Asymptotics for Local-Stochastic Volatility Model}

Consider the following diffusion:

$$
\begin{aligned}
d X_{t} & =\sigma_{t} c\left(X_{t}\right) d W_{t} \\
X_{0} & =x_{0}>0, \\
d \sigma_{t} & =a\left(\sigma_{t}\right) d t+b\left(\sigma_{t}\right) d Z_{t}, \\
\sigma_{0} & =\sigma>0,
\end{aligned}
$$

where $W_{t}$ and $Z_{t}$ are two standard Brownian motions with correlation $\rho$. 
Proposition 6.3 When $t \downarrow 0$, we have

$$
p\left(t, x_{0}, x\right) \sim \frac{1}{\sqrt{2 \pi \sigma_{0}^{2} c\left(x_{0}\right)^{2} t}} \exp \left(-\frac{\left(x_{0}-x\right)^{2}}{2 \sigma_{0}^{2} c\left(x_{0}\right)^{2} t}\right)\left(1+\sqrt{t} \eta\left(t, x_{0}, x\right)\right),
$$

where

$$
\eta\left(t, x_{0}, x\right)=\frac{1}{2}\left(\rho b(\sigma)+\sigma^{2} \partial c\left(x_{0}\right)\right) \sigma^{2} c\left(x_{0}\right)^{3}\left\{\frac{h_{3}\left(\left(x-x_{0}\right) / \sqrt{t}, \sigma^{2} c\left(x_{0}\right)^{2}\right)}{\left(\sigma^{2} c\left(x_{0}\right)^{2}\right)^{3}}\right\} .
$$

Proof 6.3 We compute the coefficient of the first order in the expansion by applying (5.17) and (5.26).

First, we introduce the time scaling parameter $\epsilon=\sqrt{t}$,

$$
\begin{aligned}
d X_{t} & =\epsilon \sigma_{t} c\left(X_{t}\right) d W_{t}, \\
d \sigma_{t} & =\epsilon^{2} a\left(\sigma_{t}\right) d t+\epsilon b\left(\sigma_{t}\right) d Z_{t} .
\end{aligned}
$$

The generator $\mathcal{A}^{\epsilon}$ associated with $X$ is given by

$$
\mathcal{A}^{\epsilon}=\epsilon^{2} \frac{1}{2} \sigma^{2} c(x)^{2} \frac{\partial^{2}}{\partial x^{2}}+\epsilon^{2} \rho \sigma b(\sigma) c(x) \frac{\partial^{2}}{\partial x \partial \sigma}+\epsilon^{2} a(\sigma) \frac{\partial}{\partial \sigma}+\epsilon^{2} \frac{1}{2} b(\sigma)^{2} \frac{\partial^{2}}{\partial \sigma^{2}} .
$$

When $\epsilon \downarrow 0, \mathcal{A}^{\epsilon}$ is degenerate. We consider the following transform,

$$
Y=\frac{X-x_{0}}{\epsilon}
$$

Then the generator $\mathcal{L}^{\epsilon}$ associated with $Y$ is elliptic under $\epsilon \downarrow 0$ and is given by

$$
\mathcal{L}^{\epsilon}=\frac{1}{2} \sigma^{2} c\left(x_{0}+\epsilon y\right)^{2} \frac{\partial^{2}}{\partial y^{2}}+\epsilon \rho \sigma b(\sigma) c\left(x_{0}+\epsilon y\right) \frac{\partial^{2}}{\partial y \partial \sigma}+\epsilon^{2} a(\sigma) \frac{\partial}{\partial \sigma}+\epsilon^{2} \frac{1}{2} b(\sigma)^{2} \frac{\partial^{2}}{\partial \sigma^{2}},
$$

and

$$
u^{\epsilon}\left(t, y_{0}\right)=\mathbf{P}_{t}^{\epsilon} f\left(y_{0}\right)=\int_{\mathbf{R}} f(y) p^{Y^{\epsilon}}\left(t, y_{0}, d y\right)
$$

is the fundamental solution to the following equation,

$$
\begin{aligned}
\left(\frac{\partial}{\partial t}-\mathcal{L}^{\epsilon}\right) u^{\epsilon}\left(t, y_{0}\right) & =0 \\
u^{\epsilon}\left(t_{0}, y_{0}\right) & =f\left(y_{0}\right) .
\end{aligned}
$$

By differentiating (6.45) at $t=1$, we have

$$
\left.\frac{\partial}{\partial \epsilon}\right|_{\epsilon=0} \mathbf{P}_{1}^{\epsilon} f\left(y_{0}\right)=\int_{\mathbf{R}} f(y) w_{1}(y) p^{Y^{\epsilon}}\left(1, y_{0}, y\right) d y,
$$

where the map $y \mapsto w_{1}(y)$ is the first order PDE weight.

1. Using the Malliavin-Bismut-Léandre's formula, we derive the first order PDE weight $w_{1}(y)$,

$$
\begin{aligned}
\left.\frac{\partial}{\partial \epsilon}\right|_{\epsilon=0} \mathbf{P}_{1}^{\epsilon} f\left(y_{0}\right) & =\int_{0}^{1} \mathbf{P}_{(1-s)}^{0} \mathcal{L}^{1} \mathbf{P}_{s}^{0} f\left(y_{0}\right) d s \\
& =\int_{\mathbf{R}} f(y) \frac{1}{\sigma^{2} c\left(x_{0}\right)^{2}} E\left[Y_{11} \int_{0}^{1} \sigma c\left(x_{0}\right) d W_{1, t}-\int_{0}^{1} D_{t, 1} Y_{11} \sigma c\left(x_{0}\right) d t \mid Y_{01}=y\right] p\left(1, y_{0}, y\right) d y
\end{aligned}
$$

$\mathcal{L}^{0}$ and $\mathcal{L}^{1}$ are given as follows;

$$
\begin{aligned}
\mathcal{L}^{0} & =\frac{1}{2} \sigma^{2} c\left(x_{0}\right)^{2} \frac{\partial^{2}}{\partial y^{2}} \\
\mathcal{L}^{1} & =\sigma^{2} c\left(x_{0}\right) \partial c\left(x_{0}\right) y \frac{\partial^{2}}{\partial y^{2}}+\rho \sigma b(\sigma) c\left(x_{0}\right) \frac{\partial^{2}}{\partial y \partial \sigma} .
\end{aligned}
$$


Note that

$$
\begin{aligned}
\frac{\partial}{\partial \sigma} p^{Y^{0}}(s, y, z) & =\sigma s c\left(x_{0}\right)^{2} \frac{\partial^{2}}{\partial y^{2}} p^{Y^{0}}(s, y, z), \\
p^{Y^{0}}\left(1-s, y_{0}, y\right) y & =\left.(1-s) \sigma^{2} c\left(x_{0}\right)^{2} \frac{\partial}{\partial y_{0}} p^{Y^{0}}\left(1-s, y_{0}, y\right)\right|_{y_{0}=0} .
\end{aligned}
$$

Let $g$ be a map $y \mapsto g(y)$ such that

$$
\begin{aligned}
g(y) & =\mathcal{L}^{1} \mathbf{P}_{s} f(y)=\mathcal{L}^{1} \mathbf{P}_{1-(1-s)} f(y) \\
& =\mathcal{L}^{1} E\left[f\left(Y_{1}^{0}\right) \mid Y_{1-s}^{0}=y\right]=\mathcal{L}^{1} \int_{\mathbf{R}} p^{Y^{0}}(s, y, z) f(z) d z
\end{aligned}
$$

Then, we explicitly evaluate (5.26) for $j=1$.

$$
\begin{aligned}
& \left.\mathbf{P}_{(1-s)}^{0} \mathcal{L}^{1} \mathbf{P}_{s}^{0} f\left(y_{0}\right)\right|_{y_{0}=0} \\
= & \left.\int_{\mathbf{R}} p^{Y^{0}}\left(1-s, y_{0}, y\right) g(y) d y\right|_{y_{0}=0} \\
= & \left.\int_{\mathbf{R}} p^{Y^{0}}\left(1-s, y_{0}, y\right)\left(\mathcal{L}^{1} \int_{\mathbf{R}} p^{Y^{0}}(s, y, z) f(z) d z\right) d y\right|_{y_{0}=0} \\
= & \left.\int_{\mathbf{R}}\left(\int_{\mathbf{R}}(1-s) \sigma^{4} c\left(x_{0}\right)^{3} \partial c\left(x_{0}\right)\left(\frac{\partial}{\partial y_{0}} p^{Y^{0}}\left(1-s, y_{0}, y\right)\right) \frac{\partial^{2}}{\partial y^{2}} p^{Y^{0}}(s, y, z) d y\right) f(z) d z\right|_{y_{0}=0} \\
& +\left.\int_{\mathbf{R}}\left(\int_{\mathbf{R}} p^{Y^{0}}\left(1-s, y_{0}, y\right) s \rho \sigma^{2} b(\sigma) c\left(x_{0}\right)^{3} \frac{\partial^{3}}{\partial y^{3}} p^{Y^{0}}(s, y, z) d y\right) f(z) d z\right|_{y_{0}=0} \\
= & \left.\int_{\mathbf{R}}\left(\int_{\mathbf{R}}(1-s) \sigma^{4} c\left(x_{0}\right)^{3} \partial c\left(x_{0}\right)\left(\frac{\partial^{3}}{\partial y^{2} \partial y_{0}} p^{Y^{0}}\left(1-s, y_{0}, y\right)\right) p^{Y^{0}}(s, y, z) d y\right) f(z) d z\right|_{y_{0}=0} \\
& +\left.\int_{\mathbf{R}}\left(\int_{\mathbf{R}}\left(-\frac{\partial^{3}}{\partial y^{3}} p^{Y^{0}}\left(1-s, y_{0}, y\right)\right) s \rho \sigma^{2} b(\sigma) c\left(x_{0}\right)^{3} p^{Y^{0}}(s, y, z) d y\right) f(z) d z\right|_{y_{0}=0} \\
= & \left.(1-s) \sigma^{4} c\left(x_{0}\right)^{3} \partial c\left(x_{0}\right) \frac{\partial^{3}}{\partial y_{0}^{3}} \int_{\mathbf{R}}\left(\int_{\mathbf{R}} p^{Y^{0}}\left(1-s, y_{0}, y\right) p^{Y^{0}}(s, y, z) d y\right) f(z) d z\right|_{y_{0}=0} \\
& +\left.s \rho \sigma^{2} b(\sigma) c\left(x_{0}\right)^{3} \frac{\partial^{3}}{\partial y_{0}^{3}} \int_{\mathbf{R}}\left(\int_{\mathbf{R}} p^{Y^{0}}\left(1-s, y_{0}, y\right) p^{Y^{0}}(s, y, z) d y\right) f(z) d z\right|_{y_{0}=0} \\
= & (1-s) \sigma^{4} c\left(x_{0}\right)^{3} \partial c\left(x_{0}\right) \frac{\partial^{3}}{\partial y_{0}^{3}} \int_{\mathbf{R}} p^{Y^{0}}\left(1, y_{0}, z\right) f(z) d z+\left.s \rho \sigma^{2} b(\sigma) c\left(x_{0}\right)^{3} \frac{\partial^{3}}{\partial y_{0}^{3}} \int_{\mathbf{R}} p^{Y^{0}}\left(1, y_{0}, z\right) f(z) d z\right|_{y_{0}=0} \\
= & \left.(1-s) \sigma^{4} c\left(x_{0}\right)^{3} \partial c\left(x_{0}\right) \frac{\partial^{3}}{\partial y_{0}^{3}} \mathbf{P}_{1} f\left(y_{0}\right)\right|_{y_{0}=0}+\left.s \rho \sigma^{2} b(\sigma) c\left(x_{0}\right)^{3} \frac{\partial^{3}}{\partial y_{0}^{3}} \mathbf{P}_{1} f\left(y_{0}\right)\right|_{y_{0}=0} . \\
& \\
&
\end{aligned}
$$

Therefore, we have

$$
\begin{aligned}
& \left.\int_{0}^{1} \mathbf{P}_{(1-s)}^{0} \mathcal{L}^{1} \mathbf{P}_{s}^{0} f\left(y_{0}\right) d s\right|_{y_{0}=0} \\
= & \left.\frac{1}{2}\left(\sigma^{4} c\left(x_{0}\right)^{3} \partial c\left(x_{0}\right) \frac{\partial^{3}}{\partial y_{0}^{3}}+\rho \sigma^{2} b(\sigma) c\left(x_{0}\right)^{3} \frac{\partial^{3}}{\partial y_{0}^{3}}\right) \mathbf{P}_{1}^{0} f\left(y_{0}\right)\right|_{y_{0}=0} .
\end{aligned}
$$

and

$$
\begin{aligned}
\left.\frac{\partial}{\partial \epsilon}\right|_{\epsilon=0} p^{Y^{\epsilon}}\left(1, y_{0}, y\right) & =\frac{1}{2}\left(\sigma^{4} c\left(x_{0}\right)^{3} \partial c\left(x_{0}\right) \frac{\partial^{3}}{\partial y_{0}^{3}}+\rho \sigma^{2} b(\sigma) c\left(x_{0}\right)^{3} \frac{\partial^{3}}{\partial y_{0}^{3}}\right) p^{Y^{0}}\left(1, y_{0}, y\right) \\
& =\frac{1}{2}\left(\rho b(\sigma)+\sigma^{2} \partial c\left(x_{0}\right)\right) \sigma^{2} c\left(x_{0}\right)^{3} \frac{h_{3}\left(y, \sigma^{2} c\left(x_{0}\right)^{2}\right)}{\left(\sigma^{2} c\left(x_{0}\right)^{2}\right)^{3}} p^{Y^{0}}\left(1, y_{0}, y\right)
\end{aligned}
$$

Setting $y_{0}=0$, we obtain the result.

2. Next, we compute the first order PDE weight by applying (5.17) for $j=1$ in the following way. First, $X$ is approximated by stochastic Taylor expansion,

$$
X_{t}=x_{0}+X_{1 t}+X_{2 t}+R_{3}(t),
$$


where

$$
\begin{aligned}
X_{1 t} & =\int_{0}^{t} \sigma c\left(x_{0}\right) d W_{s} \\
X_{2 t} & =\int_{0}^{t} c\left(x_{0}\right) \int_{0}^{s} b(\sigma) d Z_{u} d W_{s}+\int_{0}^{t} \sigma \partial c\left(x_{0}\right) \int_{0}^{s} \sigma c\left(x_{0}\right) d W_{u} d W_{s} .
\end{aligned}
$$

$X^{(\epsilon)}$ and $Y^{(\epsilon)}$ are expanded in $\mathbf{D}_{\infty}$,

$$
\begin{aligned}
& X_{t}^{(\epsilon)}=x_{0}+\epsilon X_{1 t}+\epsilon^{2} X_{2 t}+O\left(\epsilon^{3}\right), \\
& Y_{t}^{(\epsilon)}=Y_{0 t}+\epsilon Y_{1 t}+O\left(\epsilon^{2}\right),
\end{aligned}
$$

where

$$
\begin{gathered}
Y_{0 t}=X_{1 t}=\left.\frac{\partial}{\partial \epsilon} X_{t}^{\epsilon}\right|_{\epsilon=0}=\int_{0}^{t} \sigma c\left(x_{0}\right) d W_{s} \\
Y_{1 t}=X_{2 t}=\left.\frac{1}{2} \frac{\partial^{2}}{\partial \epsilon^{2}} X_{t}^{\epsilon}\right|_{\epsilon=0}=\int_{0}^{t} c\left(x_{0}\right) \int_{0}^{s} b(\sigma) d Z_{u} d W_{s}+\int_{0}^{t} \sigma \partial c\left(x_{0}\right) \int_{0}^{s} \sigma c\left(x_{0}\right) d W_{u} d W_{s}
\end{gathered}
$$

Then, the PDE weight is caluculated as follows,

$$
\begin{aligned}
w_{1}(y) & =\frac{1}{\sigma^{2} c\left(x_{0}\right)^{2}} E\left[Y_{11} \int_{0}^{1} \sigma c\left(x_{0}\right) d W_{1, t}-\int_{0}^{1} D_{t, 1} Y_{11} \sigma c\left(x_{0}\right) d t \mid Y_{01}=y\right] \\
& =\left(\frac{y}{\sigma^{2} c\left(x_{0}\right)^{2}}-\frac{\partial}{\partial y}\right)\left(\rho \sigma^{2} b(\sigma) c\left(x_{0}\right)^{3} \int_{0}^{t} \int_{0}^{s} d u d s \frac{h_{2}\left(y, \sigma^{2} c\left(x_{0}\right)^{2}\right)}{\left(\sigma^{2} c\left(x_{0}\right)^{2}\right)^{2}}+\sigma^{4} c\left(x_{0}\right)^{3} \partial c\left(x_{0}\right) \int_{0}^{t} \int_{0}^{s} d u d s \frac{h_{2}\left(y, \sigma^{2} c\left(x_{0}\right)^{2}\right)}{\left(\sigma^{2} c\left(x_{0}\right)^{2}\right)^{2}}\right) \\
& =\left(\left(\frac{1}{2} \rho \sigma^{2} b(\sigma) c\left(x_{0}\right)^{3} \frac{h_{3}\left(y, \sigma^{2} c\left(x_{0}\right)^{2}\right)}{\left(\sigma^{2} c\left(x_{0}\right)^{2}\right)^{3}}+\frac{1}{2} \sigma^{4} c\left(x_{0}\right)^{3} \partial c\left(x_{0}\right) \frac{h_{3}\left(y, \sigma^{2} c\left(x_{0}\right)^{2}\right)}{\left.\left.\left(\sigma^{2} c\left(x_{0}\right)^{2}\right)^{3}\right)\right)}\right.\right. \\
& =\frac{1}{2}\left(\rho b(\sigma)+\sigma^{2} \partial c\left(x_{0}\right)\right) \sigma^{2} c\left(x_{0}\right)^{3} \frac{h_{3}\left(y, \sigma^{2} c\left(x_{0}\right)^{2}\right)}{\left(\sigma^{2} c\left(x_{0}\right)^{2}\right)^{3}} .
\end{aligned}
$$

The following formula holds,

$$
p^{\epsilon}\left(1, x_{0}, x\right)=p(1,0, f(x)) \frac{1}{\epsilon}
$$

and we have

$$
p\left(t, x_{0}, x\right)=p^{\sqrt{t}}\left(1, x_{0}, x\right) .
$$

Then, we obtain a short time off-diagonal asymptotic expansion of heat kernel,

$$
p\left(t, x_{0}, x\right) \sim \frac{1}{\sqrt{2 \pi \sigma_{0}^{2} c\left(x_{0}\right)^{2} t}} \exp \left(-\frac{\left(x_{0}-x\right)^{2}}{2 \sigma_{0}^{2} c\left(x_{0}\right)^{2} t}\right)\left(1+\sqrt{t} \eta\left(t, x_{0}, x\right)\right),
$$

where

$$
\eta\left(t, x_{0}, x\right)=\frac{1}{2}\left(\rho b(\sigma)+\sigma^{2} \partial c\left(x_{0}\right)\right) \sigma^{2} c\left(x_{0}\right)^{3} \frac{h_{3}\left(\left(x-x_{0}\right) / \sqrt{t}, \sigma^{2} c\left(x_{0}\right)^{2}\right)}{\left(\sigma^{2} c\left(x_{0}\right)^{2}\right)^{3}}
$$

\subsection{Numerical Example}

This subsection provides an numerical example for option pricing under the short-time asymptotic expansion. In particular, we use the following Heston model:

$$
\begin{aligned}
d S_{t} & =\sqrt{v_{t}} S_{t} d W_{1, t}, \\
d v_{t} & =\kappa\left(\theta-v_{t}\right) d t+\nu \sqrt{v_{t}}\left(\rho d W_{1, t}+\sqrt{1-\rho^{2}} d W_{2, t}\right),
\end{aligned}
$$

with parameters $S_{0}=100, v_{0}=0.16, \kappa=1.0, \theta=0.16, \nu=0.1, \rho=-0.5$. 
The call option price with strike $K$ and maturity $t$ is approximated as follows;

$$
C(t, K)=E\left[\left(S_{t}-K\right)^{+}\right] \sim C_{0}(t, K)+\sqrt{t} C_{1}(t, K)+t C_{2}(t, K),
$$

where

$$
\begin{aligned}
C_{0}(t, K) & =\int_{\mathbf{R}}\left(e^{x}-K\right)^{+} p\left(t, x_{0}, x\right) d x, \\
C_{1}(t, K) & =\int_{\mathbf{R}}\left(e^{x}-K\right)^{+} w_{1}\left(t, x_{0}, x\right) p\left(t, x_{0}, x\right) d x, \\
C_{2}(t, K) & =\int_{\mathbf{R}}\left(e^{x}-K\right)^{+} w_{2}\left(t, x_{0}, x\right) p\left(t, x_{0}, x\right) d x .
\end{aligned}
$$

Here, $p\left(t, x_{0}, x\right)$ is given in (6.15), and $w_{1}\left(t, x_{0}, x\right)$ and $w_{2}\left(t, x_{0}, x\right)$ are given in the following:

$$
\begin{aligned}
w_{1}\left(t, x_{0}, x\right)= & \frac{1}{4} \rho \nu v_{0} \frac{h_{3}\left(\left(x-x_{0}\right) / \sqrt{t}, v_{0}\right)}{v_{0}^{3}}+\left(r-\frac{1}{2} v_{0}\right) \frac{h_{1}\left(\left(x-x_{0}\right) / \sqrt{t}, v_{0}\right)}{v_{0}}, \\
w_{2}\left(t, x_{0}, x\right)= & \frac{1}{32} \nu^{2} v_{0}^{2} \rho^{2} \frac{h_{6}\left(\left(x-x_{0}\right) / \sqrt{t}, v_{0}\right)}{v_{0}^{6}} \\
& +\left(\frac{1}{4}\left(r-\frac{1}{2} v_{0}\right) \rho \nu v_{0}+\frac{1}{12} \nu^{2} v_{0} \rho^{2}+\frac{1}{36} \rho^{2} \nu \sqrt{v_{0}} v_{0}\right) \frac{h_{4}\left(\left(x-x_{0}\right) / \sqrt{t}, v_{0}\right)}{v_{0}^{4}} \\
& +\left(\frac{1}{16} \nu^{2} v_{0} \rho^{2}+\frac{1}{2}\left(r-\frac{1}{2} v_{0}\right)^{2}+\frac{1}{12} \kappa\left(\theta-v_{0}\right)-\frac{1}{4} \rho \nu^{2} v_{0} \sqrt{v_{0}}-\frac{1}{16} \nu^{2} \rho^{2}\right) \frac{h_{2}\left(\left(x-x_{0}\right) / \sqrt{t}, v_{0}\right)}{v_{0}^{2}} .
\end{aligned}
$$

Table 2: Short time asymptotics $T=0.1$

\begin{tabular}{|l|r|rrr|rrr|}
\hline Strike & Benchmark & HKE order 2 & HKE order 1 & HKE order 0 & Error order 2 & Error order 1 & Error order 0 \\
\hline \hline 70 & 30.01 & 30.01 & 30.00 & 30.81 & $-0.01 \%$ & $-0.02 \%$ & $2.67 \%$ \\
80 & 20.19 & 20.19 & 20.18 & 20.95 & $-0.01 \%$ & $-0.04 \%$ & $3.78 \%$ \\
90 & 11.38 & 11.38 & 11.37 & 12.02 & $-0.04 \%$ & $-0.11 \%$ & $5.60 \%$ \\
100 & 5.04 & 5.03 & 5.02 & 5.47 & $-0.14 \%$ & $-0.30 \%$ & $8.66 \%$ \\
110 & 1.70 & 1.69 & 1.68 & 1.93 & $-0.37 \%$ & $-0.84 \%$ & $13.95 \%$ \\
120 & 0.44 & 0.43 & 0.43 & 0.53 & $-0.80 \%$ & $-2.33 \%$ & $22.82 \%$ \\
130 & 0.09 & 0.09 & 0.08 & 0.12 & $-1.38 \%$ & $-6.04 \%$ & $36.91 \%$ \\
\hline
\end{tabular}

Table 3: Short time asymptotics $T=0.2$

\begin{tabular}{|l|r|rrr|rrr|}
\hline Strike & Benchmark & HKE order 2 & HKE order 1 & HKE order 0 & Error order 2 & Error order 1 & Error order 0 \\
\hline \hline 70 & 30.15 & 30.14 & 30.12 & 31.72 & $-0.03 \%$ & $-0.09 \%$ & $5.19 \%$ \\
80 & 20.86 & 20.84 & 20.82 & 22.29 & $-0.06 \%$ & $-0.17 \%$ & $6.88 \%$ \\
90 & 12.94 & 12.93 & 12.90 & 14.14 & $-0.13 \%$ & $-0.33 \%$ & $9.27 \%$ \\
100 & 7.12 & 7.09 & 7.07 & 8.02 & $-0.31 \%$ & $-0.66 \%$ & $12.71 \%$ \\
110 & 3.46 & 3.44 & 3.42 & 4.07 & $-0.63 \%$ & $-1.35 \%$ & $17.67 \%$ \\
120 & 1.50 & 1.48 & 1.46 & 1.87 & $-1.14 \%$ & $-2.75 \%$ & $24.73 \%$ \\
130 & 0.59 & 0.58 & 0.56 & 0.79 & $-1.84 \%$ & $-5.41 \%$ & $34.48 \%$ \\
\hline
\end{tabular}

\section{A Second Order Approximation in Section 6.1}

1. Applying Bismut identity, the weights of second order approximations are calculated as follows.

$$
\left.\frac{1}{2} \frac{\partial^{2}}{\partial \epsilon^{2}} p^{Y^{\epsilon}}(1,0, y)\right|_{\epsilon=0}=\left\{\frac{1}{2} E\left[H_{2}\left(Y_{01}, Y_{11}^{2}\right)\right]+E\left[H_{1}\left(Y_{01}, Y_{21}\right)\right]\right\} p^{Y^{0}}(1,0, y) .
$$


Table 4: Short time asymptotics $T=0.3$

\begin{tabular}{|l|r|rrr|rrr|}
\hline Strike & Benchmark & HKE order 2 & HKE order 1 & HKE order 0 & Error order 2 & Error order 1 & Error order 0 \\
\hline \hline 70 & 30.44 & 30.42 & 30.38 & 32.74 & $-0.07 \%$ & $-0.21 \%$ & $7.53 \%$ \\
80 & 21.63 & 21.61 & 21.56 & 23.71 & $-0.12 \%$ & $-0.35 \%$ & $9.60 \%$ \\
90 & 14.26 & 14.23 & 14.18 & 16.02 & $-0.24 \%$ & $-0.60 \%$ & $12.35 \%$ \\
100 & 8.70 & 8.66 & 8.61 & 10.10 & $-0.49 \%$ & $-1.06 \%$ & $16.01 \%$ \\
110 & 4.93 & 4.89 & 4.84 & 5.96 & $-0.89 \%$ & $-1.91 \%$ & $20.89 \%$ \\
120 & 2.61 & 2.58 & 2.53 & 3.33 & $-1.47 \%$ & $-3.38 \%$ & $27.30 \%$ \\
130 & 1.31 & 1.27 & 1.23 & 1.77 & $-2.24 \%$ & $-5.83 \%$ & $35.59 \%$ \\
\hline
\end{tabular}

Table 5: Short time asymptotics $T=0.4$

\begin{tabular}{|l|r|rrr|rrr|}
\hline Strike & Benchmark & HKE order 2 & HKE order 1 & HKE order 0 & Error order 2 & Error order 1 & Error order 0 \\
\hline \hline 70 & 30.83 & 30.79 & 30.71 & 33.83 & $-0.12 \%$ & $-0.37 \%$ & $9.74 \%$ \\
80 & 22.41 & 22.37 & 22.29 & 25.13 & $-0.20 \%$ & $-0.57 \%$ & $12.11 \%$ \\
90 & 15.41 & 15.35 & 15.27 & 17.74 & $-0.38 \%$ & $-0.92 \%$ & $15.11 \%$ \\
100 & 10.04 & 9.97 & 9.88 & 11.94 & $-0.68 \%$ & $-1.51 \%$ & $18.94 \%$ \\
110 & 6.21 & 6.14 & 6.06 & 7.69 & $-1.15 \%$ & $-2.49 \%$ & $23.79 \%$ \\
120 & 3.68 & 3.61 & 3.53 & 4.77 & $-1.80 \%$ & $-4.07 \%$ & $29.87 \%$ \\
130 & 2.09 & 2.03 & 1.95 & 2.87 & $-2.64 \%$ & $-6.49 \%$ & $37.41 \%$ \\
\hline
\end{tabular}

Iterating the Bismut identity, the terms of $\frac{1}{2} H_{2}\left(Y_{01}, Y_{11}^{2}\right)$ are calculated as follows:

$$
\begin{gathered}
\left.E\left[H_{2}\left(Y_{t}^{(0)}, \frac{1}{2}\left(\mu\left(x_{0}\right) t\right)^{2}\right)\right) \mid \sigma\left(x_{0}\right) W_{t}=y\right] \\
=\frac{1}{2}\left(\mu\left(x_{0}\right) t\right)^{2} \frac{h_{2}\left(y, \sigma^{2}\left(x_{0}\right)\right)}{\left(\sigma^{2}\left(x_{0}\right)\right)^{2}}, \\
E\left[H_{2}\left(Y_{t}^{(0)}, \mu\left(x_{0}\right) t \sigma\left(x_{0}\right) \partial \sigma\left(x_{0}\right) \int_{0}^{t} W_{s} d W_{s}\right) \mid \sigma\left(x_{0}\right) W_{t}=y\right] \\
=\frac{1}{2} t^{2} \mu\left(x_{0}\right) t \sigma^{3}\left(x_{0}\right) \partial \sigma\left(x_{0}\right) \frac{h_{4}\left(y, \sigma^{2}\left(x_{0}\right)\right)}{\left(\sigma^{2}\left(x_{0}\right)\right)^{4}}, \\
E\left[H_{2}\left(Y_{t}^{(0)}, \frac{1}{2}\left(\sigma\left(x_{0}\right) \partial \sigma\left(x_{0}\right)\right)^{2}\left(\int_{0}^{t} W_{s} d W_{s}\right)^{2}\right) \mid \sigma\left(x_{0}\right) W_{t}=y\right] \\
=\frac{1}{8} t^{2}\left(\partial \sigma\left(x_{0}\right)\right)^{2} \sigma^{6}\left(x_{0}\right) \frac{h_{6}\left(y, \sigma^{2}\left(x_{0}\right)\right)}{\left(\sigma^{2}\left(x_{0}\right)\right)^{6}} \\
+\frac{1}{2}\left\{t^{3}\left(\partial \sigma\left(x_{0}\right)\right)^{2} \sigma^{4}\left(x_{0}\right)\right\} \frac{h_{4}\left(y, \sigma^{2}\left(x_{0}\right)\right)}{\left(\sigma^{2}\left(x_{0}\right)\right)^{4}} \\
+\frac{1}{4} t^{2}\left(\sigma\left(x_{0}\right) \partial \sigma\left(x_{0}\right)\right)^{2} \frac{h_{2}\left(y, \sigma^{2}\left(x_{0}\right)\right)}{\left(\sigma^{2}\left(x_{0}\right)\right)^{2}} .
\end{gathered}
$$

The terms of $H_{1}\left(Y_{01}, Y_{21}\right)$ are calculated as follows,

$$
\begin{aligned}
& E\left[H_{1}\left(Y_{t}^{(0)}, \partial \mu\left(x_{0}\right) \sigma\left(x_{0}\right) \int_{0}^{t} W_{s} d s\right) \mid \sigma\left(x_{0}\right) W_{t}=y\right] \\
= & \frac{1}{2} \partial \mu\left(x_{0}\right) \sigma^{2}\left(x_{0}\right) t^{2} \frac{h_{2}\left(y, \sigma^{2}\left(x_{0}\right)\right)}{\left(\sigma^{2}\left(x_{0}\right)\right)^{2}}
\end{aligned}
$$




$$
\begin{aligned}
& E\left[H_{1}\left(Y_{t}^{(0)}, \frac{1}{2} \sigma^{2}\left(x_{0}\right) \partial^{2} \sigma\left(x_{0}\right) \int_{0}^{t} W_{s}^{2} d W_{s}\right) \mid \sigma\left(x_{0}\right) W_{t}=y\right] \\
&= \frac{1}{6} \sigma^{2}\left(x_{0}\right) \partial^{2} \sigma\left(x_{0}\right) \sigma^{3}\left(x_{0}\right) t^{3} \frac{h_{4}\left(y, \sigma^{2}\left(x_{0}\right)\right)}{\left(\sigma^{2}\left(x_{0}\right)\right)^{4}} \\
&+ \frac{1}{4} \sigma^{2}\left(x_{0}\right) \partial^{2} \sigma\left(x_{0}\right) \sigma\left(x_{0}\right) t^{2} \frac{h_{2}\left(y, \sigma^{2}\left(x_{0}\right)\right)}{\left(\sigma^{2}\left(x_{0}\right)\right)^{2}}, \\
& E\left[H_{1}\left(Y_{t}^{(0)}, \mu\left(x_{0}\right) \partial \sigma\left(x_{0}\right) \int_{0}^{t} s d W_{s}\right) \mid \sigma\left(x_{0}\right) W_{t}=y\right] \\
&=\frac{1}{2} \mu\left(x_{0}\right) \partial \sigma\left(x_{0}\right) \sigma\left(x_{0}\right) t^{2} \frac{h_{1}\left(y, \sigma^{2}\left(x_{0}\right)\right)}{\left(\sigma^{2}\left(x_{0}\right)\right)^{2}}, \\
& E\left[H_{1}\left(Y_{t}^{(0)},\left(\partial \sigma\left(x_{0}\right)\right)^{2} \sigma\left(x_{0}\right) \int_{0}^{t} \int_{0}^{s} W_{u} d W_{u} d W_{s}\right) \mid \sigma\left(x_{0}\right) W_{t}=y\right] \\
&=\frac{1}{6} \partial \sigma\left(x_{0}\right)^{2} \sigma\left(x_{0}\right)^{4} t^{3} \frac{h_{4}\left(y, \sigma^{2}\left(x_{0}\right)\right)}{\left(\sigma^{2}\left(x_{0}\right)\right)^{4}}
\end{aligned}
$$

Therefore, we obtain the second approximation term

$$
\begin{aligned}
& \left.\frac{\partial^{2}}{\partial \epsilon^{2}} E\left[\delta_{y}\left(Y_{1}^{(\epsilon)}\right)\right]\right|_{\epsilon=0} \\
= & \left\{\frac{1}{8} \partial \sigma\left(x_{0}\right)^{2} \sigma^{6}\left(x_{0}\right) \frac{\left.h_{6}\left(y, \sigma^{2}\left(x_{0}\right)\right)\right)}{\left(\sigma^{2}\left(x_{0}\right)\right)^{6}}\right. \\
& +\frac{1}{6}\left(\partial^{2} \sigma\left(x_{0}\right) \sigma\left(x_{0}\right)^{5}+4 \partial \sigma\left(x_{0}\right)^{2} \sigma\left(x_{0}\right)+3 \mu\left(x_{0}\right) \partial \sigma\left(x_{0}\right)\right) \frac{h_{4}\left(y, \sigma^{2}\left(x_{0}\right)\right)}{\left.\left(\sigma^{2}\left(x_{0}\right)\right)\right)^{4}} \\
& \left.+\frac{1}{4}\left(\partial^{2} \sigma\left(x_{0}\right) \sigma\left(x_{0}\right)^{3}+2 \mu^{\prime}\left(x_{0}\right) \sigma\left(x_{0}\right)^{2}+2 \mu\left(x_{0}\right) \partial \sigma\left(x_{0}\right) \sigma\left(x_{0}\right)+\partial \sigma\left(x_{0}\right)^{2} \sigma\left(x_{0}\right)^{2}+2 \mu\left(x_{0}\right)^{2}\right) \frac{h_{2}\left(x, \sigma^{2}\left(x_{0}\right)\right)}{\left(\sigma^{2}\left(x_{0}\right)\right)^{2}}\right\} \\
& \times p\left(1, x_{0}, y\right) .
\end{aligned}
$$

2. Using the Lie bracket, the second order term is calculated as follows.

$\left.\frac{1}{2} \frac{\partial^{2}}{\partial \epsilon^{2}}\right|_{\epsilon=0} \mathbf{P}_{t}^{\epsilon}=\left.\int_{0}^{t} \int_{0}^{t_{1}} \mathbf{P}_{\left(t-t_{1}\right)}^{0} \mathcal{L}^{1} \mathbf{P}_{\left(t_{1}-t_{2}\right)}^{0} \mathcal{L}^{1} \mathbf{P}_{t_{2}}^{0} f\left(y_{0}\right) d t_{2} d t_{1}\right|_{y_{0}=0}+\left.\int_{0}^{t} \mathbf{P}_{\left(t-t_{1}\right)}^{0} \mathcal{L}^{2} \mathbf{P}_{t_{1}}^{0} f\left(y_{0}\right) d t_{1}\right|_{y_{0}=0}$

The first term is given by

$$
\left.\int_{0}^{t} \int_{0}^{t_{1}}\left(\mathcal{L}^{1}+\left(t-t_{1}\right)\left[\mathcal{L}^{0}, \mathcal{L}^{1}\right]\right)\left(\mathcal{L}^{1}+\left(t-t_{2}\right)\left[\mathcal{L}^{0}, \mathcal{L}^{1}\right]\right) \mathbf{P}_{t}^{0} f\left(y_{0}\right) d t_{2} d t_{1}\right|_{y_{0}=0}
$$

since $\left[\mathcal{L}^{0},\left[\mathcal{L}^{0}, \mathcal{L}^{1}\right]\right]=0$.

The second term is given by

$$
\left.\int_{0}^{t}\left(\mathcal{L}^{2}+\left(t-t_{1}\right)\left[\mathcal{L}^{0}, \mathcal{L}^{2}\right]+\frac{1}{2}\left(t-t_{1}\right)^{2}\left[\mathcal{L}^{0},\left[\mathcal{L}^{0}, \mathcal{L}^{2}\right]\right]\right) \mathbf{P}_{t}^{0} f\left(y_{0}\right) d t_{1}\right|_{y_{0}=0}
$$

because $\left[\mathcal{L}^{0},\left[\mathcal{L}^{0},\left[\mathcal{L}^{0}, \mathcal{L}^{1}\right]\right]\right]=0$.

Then we have

$$
\begin{aligned}
& \left.\frac{1}{2} \frac{\partial^{2}}{\partial \epsilon^{2}}\right|_{\epsilon=0} p^{Y^{\epsilon}}(1,0, y)= \\
= & \left.\left(\mathcal{L}^{2}+\frac{1}{2}\left[\mathcal{L}^{0}, \mathcal{L}^{2}\right]+\frac{1}{6}\left[\mathcal{L}^{0},\left[\mathcal{L}^{0}, \mathcal{L}^{2}\right]\right]+\left(\mathcal{L}^{1}\right)^{2}+\frac{1}{2} \mathcal{L}^{1}\left[\mathcal{L}^{0}, \mathcal{L}^{1}\right]+\frac{1}{6}\left[\mathcal{L}^{0}, \mathcal{L}^{1}\right] \mathcal{L}^{1}+\frac{1}{8}\left[\mathcal{L}^{0}, \mathcal{L}^{1}\right]^{2}\right) p^{Y^{0}}\left(1, y_{0}, y\right)\right|_{y_{0}=0},
\end{aligned}
$$

where

$$
p^{Y^{0}}\left(1, y_{0}, y\right)=\frac{1}{\sqrt{2 \pi \sigma\left(x_{0}\right)^{2}}} e^{-\frac{1}{2}\left(\frac{y-y_{0}}{\sigma\left(x_{0}\right)}\right)^{2}}
$$


Each terms are calculated as follows.

$$
\begin{gathered}
\left.\mathcal{L}^{2} p^{Y^{0}}\left(1, y_{0}, y\right)\right|_{y_{0}=0}=0 \\
\left.\frac{1}{2}\left[\mathcal{L}^{0}, \mathcal{L}^{2}\right] p^{Y^{0}}\left(1, y_{0}, y\right)\right|_{y_{0}=0}=\left.\frac{1}{2} \sigma\left(x_{0}\right)^{2}\left(\partial \mu\left(x_{0}\right)+\frac{1}{2} \partial \sigma\left(x_{0}\right)^{2}+\frac{1}{2} \partial^{2} \sigma\left(x_{0}\right) \sigma\left(x_{0}\right)\right) \partial^{2} p^{Y^{0}}\left(1, y_{0}, y\right)\right|_{y_{0}=0} \\
\left.\frac{1}{6}\left[\mathcal{L}^{0},\left[\mathcal{L}^{0}, \mathcal{L}^{2}\right]\right] p^{Y^{0}}\left(1, y_{0}, y\right)\right|_{y_{0}=0}=\left.\frac{1}{6} \sigma\left(x_{0}\right)^{4}\left(\partial \sigma\left(x_{0}\right)^{2}+\partial^{2} \sigma\left(x_{0}\right) \sigma\left(x_{0}\right)\right) \partial^{4} p^{Y^{0}}\left(1, y_{0}, y\right)\right|_{y_{0}=0}, \\
\left.\left(\mathcal{L}^{1}\right)^{2} p\left(1, y_{0}, y\right)\right|_{y_{0}=0}=\left.\frac{1}{2}\left(\partial \sigma\left(x_{0}\right) \sigma\left(x_{0}\right) \mu\left(x_{0}\right)+\mu\left(x_{0}\right)^{2}\right) \partial^{2} p^{Y^{0}}\left(1, y_{0}, y\right)\right|_{y_{0}=0} \\
\left.\frac{1}{2} \mathcal{L}^{1}\left[\mathcal{L}^{0}, \mathcal{L}^{1}\right] p^{Y^{0}}\left(1, y_{0}, y\right)\right|_{y_{0}=0}=\left.\frac{1}{3} \mu\left(x_{0}\right) \sigma\left(x_{0}\right)^{3} \partial \sigma\left(x_{0}\right) \partial^{4} p^{Y^{0}}\left(1, y_{0}, y\right)\right|_{y_{0}=0}, \\
\left.\frac{1}{6}\left[\mathcal{L}^{0}, \mathcal{L}^{1}\right] \mathcal{L}^{1} p^{Y^{0}}\left(1, y_{0}, y\right)\right|_{y_{0}=0}=\left.\frac{1}{6}\left(\mu\left(x_{0}\right)+3 \sigma\left(x_{0}\right) \partial \sigma\left(x_{0}\right)\right) \sigma\left(x_{0}\right)^{3} \partial \sigma\left(x_{0}\right) \partial^{4} p^{Y^{0}}\left(1, y_{0}, y\right)\right|_{y_{0}=0} \\
\left.\frac{1}{8}\left[\mathcal{L}^{0}, \mathcal{L}^{1}\right]^{2} p^{Y^{0}}\left(1, y_{0}, y\right)\right|_{y_{0}=0}=\left.\frac{1}{8} \sigma\left(x_{0}\right)^{6} \partial \sigma\left(x_{0}\right)^{2} \partial^{6} p^{Y^{0}}\left(1, y_{0}, y\right)\right|_{y_{0}=0}
\end{gathered}
$$

Hence, we have

$$
\begin{aligned}
& \left.\frac{1}{2} \frac{\partial^{2}}{\partial \epsilon^{2}}\right|_{\epsilon=0} p^{Y^{\epsilon}}(1,0, y) \\
= & \left\{\frac{1}{8} \partial \sigma\left(x_{0}\right)^{2} \sigma^{6}\left(x_{0}\right) \frac{h_{6}\left(y, \sigma^{2}\left(x_{0}\right)\right)}{\left(\sigma^{2}\left(x_{0}\right)\right)^{6}}\right. \\
& +\frac{1}{6}\left(\partial^{2} \sigma\left(x_{0}\right) \sigma\left(x_{0}\right)^{5}+4 \partial \sigma\left(x_{0}\right)^{2} \sigma\left(x_{0}\right)+3 \mu\left(x_{0}\right) \partial \sigma\left(x_{0}\right)\right) \frac{h_{4}\left(y, \sigma^{2}\left(x_{0}\right)\right)}{\left(\sigma^{2}\left(x_{0}\right)\right)^{4}} \\
& \left.+\frac{1}{4}\left(\partial^{2} \sigma\left(x_{0}\right) \sigma\left(x_{0}\right)^{3}+2 \mu^{\prime}\left(x_{0}\right) \sigma\left(x_{0}\right)^{2}+2 \mu\left(x_{0}\right) \partial \sigma\left(x_{0}\right) \sigma\left(x_{0}\right)+\partial \sigma\left(x_{0}\right)^{2} \sigma\left(x_{0}\right)^{2}+2 \mu\left(x_{0}\right)^{2}\right) \frac{h_{2}\left(y, \sigma^{2}\left(x_{0}\right)\right)}{\left(\sigma^{2}\left(x_{0}\right)\right)^{2}}\right\} \\
& \times p^{Y^{0}}(1,0, y) .
\end{aligned}
$$

Therefore, we obtain the result.

\section{References}

[1] Baudoin, F.: Stochastic Taylor Expansions and Heat Kernel Asymptotics. (2009)

[2] Ben Arous, G. and Laurence, P.: Second order expansion for implied volatility in two factor local stochastic volatility models and applications to the dynamic $\lambda$-SABR model.(2009)

[3] Black, F. and Scholes, M. (1973). : The Pricing of Options and Corporate Liabilities, Journal of Political Economy 81 (3): 637-654. (1973)

[4] Cheng, W., Costanzino,N., Liechty,J., Mazzucato,A. and Nistor,V.: Closed-form Asymptotics and Numerical Approximations of 1D Paraolic Equations with Applications to Option Pricing, Preprint, (2010).

[5] Cheng, W., Mazzucato,A. and Nistor,V.: Approximate Solutions to Second Order Parabolic Equations II: Time-dependent Coefficients, Preprint, (2011).

[6] Fouque, J. P., Papanicolaou G. and Sircar K.R., Mean Reverting Stochastic Volatility, International Journal of Theoretical and Applied Finance, 3 (2000a) 101-142.

[7] Fouque, J. P., Papanicolaou G. and Sircar K.R.: Derivatives in Financial Markets with Stochastic Volatility, Cambridge University Press (2000b). 
[8] Ilhan A., Jonsson, M. and Sircar, K.R.: Singular Perturbations for Boundary Value Problems arising from Exotic Options, SIAM Journal on Applied Mathematics, 64 (2004) 1268-1293.

[9] Fournié, E., Lasry, J.-M., Lebuchoux, J., Lions, P.-L. and Touzi, N. : Applications of Malliavin calculus to Monte-Carlo methods in Finance, Finance Stoch. 3 (1999), no. 4, 391-412.

[10] Fujii,M. and Takahashi,A.: Analytical Approximation for Non-linear FBSDEs with Perturbation Scheme, Working paper, CARF-F-248, University of Tokyo (2011).

[11] Gatheral, J., Hsu, E.P. and Laurence, P., Ouyang, C. and Wang, T-H.: Asymptotics of implied volatility in local volatility models: forthcoming in Mathematical Finance (2009)

[12] Hagan, P.S., Kumar, D., Lesniewskie, A.S., and Woodward, D.E.: Managing Smile Risk, Wilmott magazine, (2002).

[13] Ikeda, N., and Watanabe, S.: Stochastic Differential Equations and Diffusion Processes, Second Edition, North-Holland/Kodansha. (1989)

[14] Labordere, P.H.: Analysis, Geometry and Modeling in Finance : Advanced Methods in Options Pricing, Chapman and Hall, (2008).

[15] Léandre, R.: Malliavin Calculus of Bismut type without probability. (2006)

[16] Léandre, R.: Malliavin Calculus of Bismut type in semi-group theory. (2008)

[17] Malliavin, P. : Stochastic Analysis, Springer. (1997)

[18] Malliavin, P. and Thalmaier, A.: Stochastic Calculus of Variations in Mathematical Finance, Springer. (2006)

[19] Merton, R. C. :Theory of Rational Option Pricing, Bell Journal of Economics and Management Science (The RAND Corporation) 4 (1): 141-183. (1973)

[20] Takahashi, A., Takehara, K. and Toda, M.: Computation in an Asymptotic Expansion Method, Preprint, Working paper, CARF-F-149, University of Tokyo.(2009)

[21] Takahashi, A. and Yamada,T.: An Asymptotic Expansion with Push-Down of Malliavin Weights, CARF Working Paper Series CARF-F-194, University of Tokyo.(2009)

[22] Takahashi, A.: On an Asymptotic Expansion Approach to Numerical Problems in Finance, American Mathematical Society . (2009)

[23] Watanabe, S.: Analysis of Winer Functionals(Malliavin Calculus) and its Applications to Heat Kernels, The Annals pf Probability, Vol.15-1, 1-39. (1989) 